\title{
Portfolios in Stochastic Local Search: Efficiently Computing Most Probable Explanations in Bayesian Networks
}

\author{
Ole J. Mengshoel • Dan Roth • David C. Wilkins
}

Received: 4 April 2009/ Accepted: 9 March 2010

(C) Springer Science+Business Media B.V. 2010

\begin{abstract}
Portfolio methods support the combination of different algorithms and heuristics, including stochastic local search (SLS) heuristics, and have been identified as a promising approach to solve computationally hard problems. While successful in experiments, theoretical foundations and analytical results for portfolio-based SLS heuristics are less developed. This article aims to improve the understanding of the role of portfolios of heuristics in SLS. We emphasize the problem of computing most probable explanations (MPEs) in Bayesian networks (BNs). Algorithmically, we discuss a portfolio-based SLS algorithm for MPE computation, Stochastic Greedy Search (SGS). SGS supports the integration of different initialization operators (or initialization heuristics) and different search operators (greedy and noisy heuristics), thereby enabling new analytical and experimental results. Analytically, we introduce a novel Markov chain model tailored to portfolio-based SLS algorithms including SGS, thereby enabling us to analytically form expected hitting time results that explain empirical run time results. For a specific BN, we show the benefit of using a homogenous initialization portfolio. To further illustrate the portfolio approach, we consider novel additive search heuristics for handling determinism in the form of zero entries in conditional probability tables in BNs. Our additive approach adds rather than multiplies probabilities when computing the utility of an explanation.
\end{abstract}

O. J. Mengshoel $(\bowtie)$

Silicon Valley Campus, Carnegie Mellon University, NASA-Ames Research Center, Mail Stop 269-3, Bldg. T35-B, Rm. 107, P.O. Box 1, Moffett Field, CA 94035-0001, USA e-mail: ole.mengshoel@sv.cmu.edu

D. Roth

Department of Computer Science, University of Illinois at Urbana-Champaign, 3322 Siebel Center, 201 N. Goodwin Avenue, Urbana, IL 61801, USA

e-mail: danr@cs.uiuc.edu

D. C. Wilkins

Symbolic Systems Program, Stanford University, Margaret Jacks Hall, Bldg. 460, Rm. 127, Stanford, CA 94305, USA

e-mail: dwilkins@stanford.edu 
We motivate the additive measure by studying the dramatic impact of zero entries in conditional probability tables on the number of zero-probability explanations, which again complicates the search process. We consider the relationship between MAXSAT and MPE, and show that additive utility (or gain) is a generalization, to the probabilistic setting, of MAXSAT utility (or gain) used in the celebrated GSAT and WalkSAT algorithms and their descendants. Utilizing our Markov chain framework, we show that expected hitting time is a rational function-i.e. a ratio of two polynomials - of the probability of applying an additive search operator. Experimentally, we report on synthetically generated BNs as well as BNs from applications, and compare SGS's performance to that of Hugin, which performs BN inference by compilation to and propagation in clique trees. On synthetic networks, SGS speeds up computation by approximately two orders of magnitude compared to Hugin. In application networks, our approach is highly competitive in Bayesian networks with a high degree of determinism. In addition to showing that stochastic local search can be competitive with clique tree clustering, our empirical results provide an improved understanding of the circumstances under which portfoliobased SLS outperforms clique tree clustering and vice versa.

Keywords Stochastic local search • Portfolios • Bayesian networks • Most probable explanations • Determinism • Stochastic greedy search • Markov chains $\cdot$ Hitting times

\section{Introduction}

In this article we study the problem of computing a most probable explanation (MPE) in Bayesian networks [71]. Informally, an MPE is an instantiation of all nonevidence nodes in a $\mathrm{BN}$ such that no other instantiation has greater probability. MPE computation is a problem that is common to probabilistic formulations of diagnosis, image processing, error correction decoding, and genetic linkage analysis [22, 23, 49, 81, 82]. Ideally, one would prefer to use exact algorithms for MPE computation-for example algorithms such as clique tree clustering [2, 45, 79], conditioning [37, 70, 71], variable elimination [47, 85], or branch-and-bound [5052]. However, the MPE problem is computationally hard [1,80], and this hardness manifests itself in slow execution times even in relatively simple networks that are used in current applications. The complexity of structure-based algorithms depends on the treewidth of a BN's underlying graph or the optimal maximal clique size of a BN's induced clique tree $[3,16,18]$. Due to their very large treewidths or optimal maximal clique sizes, exact algorithms have proven to be infeasible or impractical in many application BNs.

Because of the limitations of exact algorithms, along with the importance of the MPE problem in applications, the development of improved algorithms for MPE computation is of great interest. Stochastic local search (SLS) algorithms have proven to be competitive in solving computationally hard problems including satisfiability (SAT) $[27,35,75,77,78]$, the most probable explanation [39, 41, 48, 54, 60], and the maximum a posteriori (MAP) hypothesis $[67,68]$. Unfortunately, the theoretical understanding of SLS algorithms has been lagging [33], and despite recent progress $[32,57,62]$ it is clear that further advances are needed. This work is part of a larger 
research effort where the ultimate goal is the development of highly adaptive but well-understood SLS algorithms, including SLS algorithms for MPE computation in BNs. Progress on such adaptive SLS algorithms has already been made, for example in the areas of adaptive noise [20,31, 53] and learning predictive models [36, 43, 73, 84]. In these adaptive SLS algorithms, there is a need to search in the space of SLS search parameters in addition to the fundamental SLS search process for an MPE. In other words, there is a two-level or two-step process: object-level SLS search, and metal-level search for approximately optimal SLS parameters that control the objectlevel SLS search process. To make the vision of this two-level or two-step process a reality, we believe that an improved understanding of the object-level search space is essential, hence we set in this paper out to provide such improved understanding in the context of portfolio methods.

Portfolio methods support the combination of a wide range of different algorithms, and have been identified as a promising research direction [25, 26, 38, 39, $60,84]$. This article provides an improved understanding of the role of portfolios of heuristics in stochastic local search. Algorithmically, we introduce a portfolio-based stochastic local search approach which utilizes an initialization portfolio and a search portfolio. Our approach is implemented in the Stochastic Greedy Search (SGS) system; two SGS algorithms called SimpleSGS and OperatorSGS are presented in this article. The OperatorSGS algorithm is a portfolio-based [25, 38, 60, 84] approach to MPE computation using SLS. It provides a flexible and general framework for stochastic explanation initialization and search. Specifically, OPERATORSGS allows us to combine different initialization operators (or initialization heuristics) and different search operators (greedy and noisy heuristics). Given our portfolio approach, one does not need to take a winner-takes-all approach to these different heuristics or operators. Instead, one can combine (and eventually adaptively tune) them according to the problem instance and application-specific requirements at hand. Within this portfolio framework, we make progress related to the use of a variety of different initialization and search algorithm operators. We introduce a novel augmented random walk model (Definition 54) and show that it induces a Markov chain (Theorem 55), thereby enabling us to analytically form expected hitting time results that parallel empirical run time results [57]. For a specific BN, we show the benefit of using a homogenous initialization portfolio (see Definition 56 and Theorem 58).

To illustrate the portfolio approach, we consider novel heuristics for handling determinism in BNs. ${ }^{1}$ Our approach, which we here carefully relate to MAXSAT, adds rather than multiplies probabilities when computing the utility of an explanation, and we therefore call it additive utility. Quantitatively, we study the impact of zero entries in CPTs on the number of zero-probability explanations, and show

\footnotetext{
${ }^{1}$ There are several alternative, very different, approaches to handling determinism. Arithmetic circuits handle determinism very well $[4,7]$, as does branch-and-bound that explores an AND/OR search tree [50-52], and one can also carefully encode a BN into a weighted MAXSAT problem instance [66, 74] and then use a weighted MAXSAT solver [19, 29, 42, 44, 46, 83]. Since the main emphasis in this article is on the portfolio approach to SLS, with determinism handling serving as an illustration, we leave detailed comparison to these alternative approaches to future work.
} 
dramatic increases in the probability of a randomly picked $\mathrm{BN}$ explanation being zero as a function of the probability of a CPT entry being zero, the number of BN nodes, and the BN node state space size (Theorem 13 ). Gain (i.e., change in utility) functions merely measure the progress made when one BN node's state is flipped in an explanation. The traditional gain function, multiplicative gain, computes change in an explanation's probability resulting from a flip. We carefully formalize and analyze multiplicative and additive gain. We consider the relationship between MAXSAT and MPE, and show that additive utility (or gain) is a generalization of MAXSAT utility (or gain) from the GSAT and WALKSAT algorithms and their descendants $[35,75,77,78]$ to the probabilistic setting. Both gain functions are utilized in greedy and noisy search operators, which make up the search portfolio, where the noisy operators are used to effectively escape local but non-global optima. Let $p_{A}$ be the probability of selecting an operator that uses additive gain. Utilizing the Markov chain framework mentioned above, we show that expected hitting time $h\left(p_{A}\right)$ is a rational function $P\left(p_{A}\right) / Q\left(p_{A}\right)$ of $p_{A}$, where $P\left(p_{A}\right)$ and $Q\left(p_{A}\right)$ are polynomials (Theorem 60).

Empirically, the performance of SGS is compared to that of the state-of-theart inference system Hugin, which implements a clique (or join) tree algorithm $[2,14,45]$. The clique tree algorithm can be used to compute either marginals [45] or MPEs [14], and is among the most well-known inference methods for Bayesian networks. We experiment with HugIN and SGS on both synthetic and application BNs. Comparisons to HugIN show that Stochastic Greedy Search performs significantly better for certain randomly generated Bayesian networks as well as for partly deterministic Bayesian networks from applications. We utilize an experimental paradigm for generating hard and easy synthetic BN instances [56, 61]. In a bipartite $\mathrm{BN}$, let $V$ be the number of root nodes and let $C$ be the number of leaf nodes. Synthetic bipartite BNs of increasing hardness can be generated by increasing $C$ while keeping $V$ constant $[54,61]$. Our results on synthetic BNs generated in this way, and where root nodes have uniform distributions and leaf node are or-nodes, are as follows: As the $C / V$-ratio increases, the measured run times of both HUGIN and SGS increase at an approximately exponential rate as a function of increasing $C / V$-ratio. However, given a suitable measure of gain, and specifically additive gain, SGS is approximately two orders of magnitude faster than Hugin. Beyond synthetic BNs, we also found that our algorithm is quite effective on application networks with substantial determinism, and in many cases it performs comparably to or better than HUGIN. In this article we highlight two reasons for SGS's success in application BNs, namely the ability to exploit different search operators in OpERATORSGS as well as the use of the additive measure of gain. (Another significant component in the success of SGS is the stochastic initialization portfolio, including its dynamic programming and forward simulation algorithms [60,62]. The heuristics in the initialization portfolio suggest good starting points to the stochastic local search component of SGS; see also [41, 67, 68].)

The rest of this article is organized as follows. Section 2 introduces the problem of computing a most probable explanation (MPE) as well as related results, definitions, and notation. Section 3 discusses measures of utility and gain which form the basis of all local search algorithms, including SGS, and pays special attention to the additive approach. In Section 4 we describe the overall structure of our stochastic local search approach, SGS. We present two SGS algorithms, namely SImPLESGS 
and OperatorSGS, and also discuss related research. Section 5 discusses analytical results. In Section 6 we turn to the experimental results of the article: Section 6.1 presents results for synthetically generated Bayesian networks, while in Section 6.2 we discuss experimental results for application BNs. Section 7 discusses related work and compares it to our two SGS variants. Section 8 concludes and discusses future work. This article extends and revises our earlier reports on SGS [54, 60].

\section{Preliminaries}

This section briefly reviews some of the key definitions and results for Bayesian networks and MPE computation as they relate to our research. Let $(\boldsymbol{X}, \boldsymbol{W})$ be a directed acyclic graph (DAG) with nodes $\boldsymbol{X}$ and edges $\boldsymbol{W}$, and let $X_{i} \in \boldsymbol{X}$. We use the notation $\Pi\left(X_{i}\right)=\Pi_{X_{i}}$ to indicate the parents of $X_{i}$ in the DAG, and $\Psi\left(X_{i}\right)=\Psi_{X_{i}}$ to indicate the children of $X_{i}$. A Bayesian network $(\mathrm{BN})$, formally introduced in Definition 1 below, represents a multi-variate probability distribution as a DAG, where the nodes represent random variables.

Definition 1 (Bayesian network) A Bayesian network is a tuple $\beta=(\boldsymbol{X}, \boldsymbol{W}, \boldsymbol{P})$, where $(\boldsymbol{X}, \boldsymbol{W})$ is a DAG with an associated set of conditional probability distributions $\boldsymbol{P}=\left\{\operatorname{Pr}\left(X_{1} \mid \Pi_{X_{1}}\right), \ldots, \operatorname{Pr}\left(X_{n} \mid \Pi_{X_{n}}\right)\right\}$. Here, $\operatorname{Pr}\left(X_{i} \mid \Pi_{X_{i}}\right)$ is the conditional probability distribution for $X_{i} \in \boldsymbol{X}$. Further, let $n=|\boldsymbol{X}|$ and let $\pi_{X_{i}}$ represent the instantiation of the parents $\Pi_{X_{i}}$ of $X_{i}$. The independence assumptions encoded in $(\boldsymbol{X}, \boldsymbol{W})$ imply the joint probability distribution

$$
\operatorname{Pr}(\boldsymbol{x})=\operatorname{Pr}\left(x_{1}, \ldots, x_{n}\right)=\operatorname{Pr}\left(X_{1}=x_{1}, \ldots, X_{n}=x_{n}\right)=\prod_{i=1}^{n} \operatorname{Pr}\left(x_{i} \mid \pi_{X_{i}}\right) .
$$

A conditional probability distribution $\operatorname{Pr}\left(X_{i} \mid \Pi_{X_{i}}\right)$ is also known as a conditional probability table (CPT). While BN nodes can be continuous, this article is restricted to the discrete case and we will take "BN node" to mean "discrete BN node". Suppose that a BN node $X$ has states $\left\{x_{1}, \ldots, x_{m}\right\}$. We then use the notation $\Omega_{X}=\Omega(X)=\left\{x_{1}, \ldots, x_{m}\right\}$. For simplicity, but without loss of generality, we often use binary nodes in the following, in which case a BN node $X$ has $\left|\Omega_{X}\right|=|X|=$ $|\{0,1\}|=2$ states.

A BN may be given evidence by clamping some of its nodes to their observed states. An instantiation of the remaining nodes is an explanation, formally defined as follows.

Definition 2 (Explanation) Consider a BN $\beta=(\boldsymbol{X}, \boldsymbol{W}, \boldsymbol{P})$ with $\boldsymbol{X}=\left\{X_{1}, \ldots, X_{n}\right\}$ and evidence $\boldsymbol{e}=\left\{X_{1}=x_{1}, \ldots, X_{m}=x_{m}\right\}$ where $m<n$. An explanation $\boldsymbol{x}$ is defined as $\boldsymbol{x}=\left\{x_{m+1}, \ldots, x_{n}\right\}=\left\{X_{m+1}=x_{m+1}, \ldots, X_{n}=x_{n}\right\}$.

When discussing an explanation $\boldsymbol{x}$, the $\mathrm{BN} \beta$ is typically left implicit. One is often interested in computing $\operatorname{Pr}(\boldsymbol{x} \mid \boldsymbol{e})$. However, in order to simplify the exposition, we may consider $\operatorname{Pr}(\boldsymbol{y})=\operatorname{Pr}(\boldsymbol{x}, \boldsymbol{e})$, where $\boldsymbol{y}=\boldsymbol{x} \cup \boldsymbol{e}$, instead of $\operatorname{Pr}(\boldsymbol{x} \mid \boldsymbol{e})$. This does not fundamentally change the computation since $\operatorname{Pr}(\boldsymbol{y})=\operatorname{Pr}(\boldsymbol{x}, \boldsymbol{e})=\operatorname{Pr}(\boldsymbol{x} \mid \boldsymbol{e}) \operatorname{Pr}(\boldsymbol{e})$. 
Given evidence, one can perform various forms of $\mathrm{BN}$ inference. This article focuses on computing the most probable explanation, which is defined as follows.

Definition 3 (Most probable explanation (MPE)) Computing a most probable explanation (MPE) in a BN with evidence $\boldsymbol{e}=\left\{X_{1}=x_{1}, \ldots, X_{m}=x_{m}\right\}$ is the problem of finding an explanation $\boldsymbol{x}^{*} \in \Omega\left(X_{m+1}\right) \times \cdots \times \Omega\left(X_{n}\right)$ such that $\operatorname{Pr}\left(\boldsymbol{x}^{*} \mid \boldsymbol{e}\right) \geq$ $\operatorname{Pr}(\boldsymbol{y} \mid \boldsymbol{e})$, where $\boldsymbol{y} \in \Omega\left(X_{m+1}\right) \times \cdots \times \Omega\left(X_{n}\right)$ is any other explanation in the BN. The set of the $k$ most probable explanations is defined as $\boldsymbol{X}^{*}=\left\{\boldsymbol{x}_{1}^{*}, \ldots, \boldsymbol{x}_{k}^{*}\right\}$ where $\operatorname{Pr}\left(\boldsymbol{x}^{*} \mid \boldsymbol{e}\right)=\operatorname{Pr}\left(\boldsymbol{x}_{1}^{*} \mid \boldsymbol{e}\right)=\cdots=\operatorname{Pr}\left(\boldsymbol{x}_{k}^{*} \mid \boldsymbol{e}\right)$.

In other words, given evidence $\boldsymbol{e}$, no other explanation has higher probability than $\boldsymbol{x}^{*} \in \boldsymbol{X}^{*}$. It is sometimes convenient to include $\boldsymbol{e}$ into an MPE and consider $\boldsymbol{y}^{*}=$ $\boldsymbol{x}^{*} \cup \boldsymbol{e}$ to be an MPE. Note that there might be many explanations with the same probability, and for this reason we say "an" MPE rather than "the" MPE.

In this article we emphasize the connection between the SAT and MPE problems and now introduce a few relevant definitions.

Definition 4 (SAT, SAT problem) A satisfiability (SAT) formula $\phi=(\boldsymbol{x}, \boldsymbol{q}, \boldsymbol{c}$ ) is defined by $V$ variables $\boldsymbol{x}=\left\{x_{1}, \ldots, x_{V}\right\}$, a set of $L$ literals $\boldsymbol{q}=\left\{q_{1}, \ldots, q_{L}\right\}$, where $q_{i}=x$ or $q_{i}=\bar{x}$ for $x \in \boldsymbol{x}$, and $C$ distinct clauses $\boldsymbol{c}=\left\{c_{1}, \ldots, c_{C}\right\}$, where each clause consists of literals combined using the or (" $\vee$ ") connective. The satisfiability (SAT) problem is to determine whether there is a truth assignment $\boldsymbol{\tau}: \boldsymbol{x} \rightarrow\{0,1\}^{V}$ that makes $c_{j}=1$ for all $1 \leq j \leq C$. Such an assignment is called a satisfying (truth) assignment (or model).

SAT is a celebrated NP-complete decision problem, and the SAT problem and algorithms for solving it are of central importance both in theoretical computer science and in artificial intelligence $[27,64,76,78]$. In a SAT problem, we have $c_{i}=1$ if the $i$-th clause is satisfied; $c_{i}=0$ if it is not satisfied. The SAT utility measure is $\prod_{i=1}^{C} c_{i}$. Consequently, $\prod_{i=1}^{C} c_{i}=1$ for some truth assignment if the formula is satisfiable, else $\prod_{i=1}^{C} c_{i}=0$ for all variable assignments.

The SAT utility measure is not very useful for hill-climbing purposes, since it does not distinguish between few and many satisfied clauses. The MAXSAT utility measure is therefore often used, and one considers the MAXSAT optimization problem.

Definition 5 (MAXSAT, MAXSAT utility) Let $\phi=(\boldsymbol{x}, \boldsymbol{q}, \boldsymbol{c})$ be a SAT formula where $C=|\boldsymbol{c}|$. The MAXSAT utility measure $U_{S}$ of $\phi$, given a truth assignment $\boldsymbol{\tau}$, is the number of true clauses in $\phi: U_{S}(\boldsymbol{\tau})=\mid\left\{c_{i} \in \boldsymbol{c} \mid c_{i}=1\right.$ and $\left.1 \leq i \leq C\right\} \mid$. The maximum satisfiability problem (MAXSAT) is to find a truth assignment $\tau^{*}$ such that the number of true clauses $U_{S}(\boldsymbol{\tau})$ in $\phi$ is maximized.

MAXSAT defines an optimization problem where one optimizes the number of satisfied clauses, $\sum_{i=1}^{C} c_{i}$. Note that $\sum_{i=1}^{C} c_{i}=C$ for some truth assignment if the formula is satisfiable, else $\sum_{i=1}^{C} c_{i}<C$ for all variable assignments. If $\sum_{i=1}^{C} c_{i}=C$ is reached in MAXSAT optimization, one has also solved the underlying SAT problem. Since SAT is NP-complete, clearly MAXSAT is NP-complete as well, however MAXSAT is much more useful for hill-climbing. A generalization of MAXSAT 
exists, called weighted MAXSAT, in which each clause $c_{i}$ has a weight $w_{i}$, and the task is to optimize the weight over all clauses. Weighted MAXSAT optimization and MPE computation are closely related; see Section 7.

A key contribution in this article, see Section 3, is how the MAXSAT utility measure can be generalized to an additive BN measure which is then utilized in MPE search. In order to set the stage we introduce these definitions.

Definition 6 (SAT-equivalent, SAT-like) A BN $\beta$ is SAT-equivalent to a SAT formula $\phi$ (and vice versa) if there is a one-to-one mapping between $\beta$ and $\phi$. A $\mathrm{BN} \beta$ is SAT-like if there exists a formula $\phi$ that is SAT-equivalent to $\beta$.

The constructions underlying Definition 6 are well-known $[8,72,80]$ and we do not discuss details in this article. Along similar lines, one can define an explanation $\boldsymbol{x}$ in a $\mathrm{BN} \beta$ as SAT-equivalent to a truth assignment $\tau$ in a formula $\phi$, where $\beta$ is SATequivalent to $\phi$ and there is a one-to-one mapping between the individual assignment of states to nodes in $\boldsymbol{x}$ and the assignments of truth values to individual variables in $\boldsymbol{\tau}$. Here, a special case of great interest occurs when an MPE $\boldsymbol{x}=\boldsymbol{x}^{*}$ is SAT-equivalent to a satisfying assignment $\boldsymbol{\tau}=\boldsymbol{\tau}^{*}$.

We now introduce a few additional SAT-related concepts. Below, $\tau$ maps one variable $x$ to $\{0,1\}$ while $\boldsymbol{\tau}$ maps all $V$ variables $\boldsymbol{x}$ in $\phi$ to $\{0,1\}^{V}$. The notation $\overline{\tau(x)}$ means that variable $x$ 's truth assignment $\tau(x)$ is inverted; $\overline{\tau(x)}=1$ iff $\tau(x)=0$ and $\overline{\tau(x)}=0$ iff $\tau(x)=1$.

Definition 7 In a SAT problem instance $\phi=(\boldsymbol{x}, \boldsymbol{q}, \boldsymbol{c})$ with truth assignment $\boldsymbol{\tau}$, let $x \in$ $\boldsymbol{x}$. The notation $\overline{\boldsymbol{\tau}}(\phi, x, \boldsymbol{\tau})$ (often abbreviated $\overline{\boldsymbol{\tau}}$ ) is a new truth assignment with $\bar{\tau}(x)=$ $\overline{\tau(x)}$ and where for all $y \in \boldsymbol{x}-\{x\}, \bar{\tau}(y)=\tau(y)$. We say that $\overline{\boldsymbol{\tau}}(\phi, x, \boldsymbol{\tau})$ is flipped compared to $\tau$.

SAT is relevant to the study of BNs for several reasons. First, BNs often have many deterministic nodes, of which the or- and and-nodes found in SAT are special cases. For examples of application BNs with many deterministic nodes, we refer to Fig. 3. Second, significant progress on stochastic local search to solve the satisfiability problem has been made in recent years $[27,35,75,77,78]$. Of particular relevance to our work is (i) the use of the GSAT measure of gain, which is based on the MAXSAT utility measure, and (ii) the use of noise as a mechanism of escape from local optima. In the research reported here, we have extended the MAXSAT utility measure and the GSAT measure of gain to the probabilistic setting, giving the additive utility measure and the additive measure of gain respectively.

It can be shown by reduction from SAT that MPE computation is NP-hard [80]. Approximating an MPE to within a constant ratio-bound has also been proven to be NP-hard [1]. In fact, the problem is much harder, since the evaluation problem is \# $P$ complete [72]. Since inference in BNs is computationally hard and the MPE problem is important in applications, we believe that it is important to study SLS algorithms for MPE computation, where estimates of $\boldsymbol{x}^{*}$ are computed.

Definition 8 (MPE (lower-bound) estimate) Let $\boldsymbol{x}^{*}$ be an MPE given evidence $\boldsymbol{e}$. A best-effort estimate of $\boldsymbol{x}^{*}$ is denoted $\hat{\boldsymbol{x}}^{*}$; if $\operatorname{Pr}\left(\hat{\boldsymbol{x}}^{*} \mid \boldsymbol{e}\right) \leq \operatorname{Pr}\left(\boldsymbol{x}^{*} \mid \boldsymbol{e}\right)$ then $\hat{\boldsymbol{x}}^{*}$ is a lowerbound estimate. 
Generally, lower-bound MPE estimates $\hat{\boldsymbol{x}}^{*}$ are computed using SLS algorithms. In the remainder of this article, our main focus is on SLS algorithms as Las Vegas algorithms [35]. In other words, we typically assume that $\operatorname{Pr}\left(\boldsymbol{x}^{*} \mid \boldsymbol{e}\right)$ or $\operatorname{Pr}\left(\boldsymbol{x}^{*}\right)$ is a known SLS input parameter, therefore SLS terminates once an $\boldsymbol{x}^{*} \in \boldsymbol{X}^{*}$ has been found. Las Vegas algorithms are used in theoretical computer science as well as in SLS research $[25,35]$, and enables our scientific study of SLS algorithms as further discussed in Section 5.1.

SLS algorithms can be analyzed using discrete time Markov chains with discrete state spaces $[32,57]$. Only time-homogenous Markov chains with finite state spaces will be considered in this article. A Markov chain is a stochastic process $\left(A_{t}, t \geq 0\right)=$ $\left(A_{0}, A_{1}, \ldots\right)$ induced by a three-tuple $\mathcal{M}=(\mathcal{S}, \mathcal{V}, \mathcal{P})$, where $\mathcal{S}$ is $k=|\mathcal{S}|$ discrete states, a $k$-dimensional vector $\mathcal{V}=\left(\pi_{1}, \ldots, \pi_{k}\right)$ represents the initial probability distribution, and a $k \times k$ matrix $\mathcal{P}$ represents the transition probabilities. As we will elaborate in Section $5, \mathcal{M}$ is given by the objective function, the SLS algorithm, and the SLS algorithm's parameter settings.

In $\mathcal{M}$, some states $\mathcal{O} \subset \mathcal{S}$ represent optimal states, and we introduce the following definition.

Definition 9 (SLS model) Let $\mathcal{M}=(\mathcal{S}, \mathcal{V}, \mathcal{P})$ define a Markov chain. Further, assume an objective function $f: \mathcal{S} \rightarrow \mathbb{R}$ and an optimal objective function value $f^{*} \in \mathbb{R}$ that defines optimal states $\mathcal{O}=\left\{s \mid s \in \mathcal{S}\right.$ and $\left.f(s)=f^{*}\right\}$. The two-tuple $(\mathcal{M}$, $\mathcal{O})$ defines an SLS model.

The objective function $f$ and the optimal states $\mathcal{O}$ are independent of the SLS algorithm and its parameters; finding an $s^{*} \in \mathcal{O}$ is the purpose of SLS search including MPE computation. We emphasize maximization in the form of MPE computation in this article, therefore $f$ in Definition 9 is given by $\operatorname{Pr}(\boldsymbol{x})$ in Definition 1 . We often consider BNs with binary nodes. In this case, explanations can be represented as bitstrings of length $n, \boldsymbol{b} \in\{0,1\}^{n}$, and $s^{*}=\boldsymbol{b}^{*} \in\{0,1\}^{n}$.

Consider an SLS model $(\mathcal{M}, \mathcal{O})$. A hitting time analysis of $\mathcal{M}$, known from Markov chain theory, gives the expected number of search steps needed to reach $s^{*} \in \mathcal{O}$. Hitting times are based on first passage times.

Definition 10 (First passage time) Consider an SLS model $(\mathcal{M}, \mathcal{O})$ and let $s_{i} \in \mathcal{S}$ where $\mathcal{S}$ is $\mathcal{M}$ 's states. The first passage time $T$, a random variable, into $s^{*} \in \mathcal{O}$ is given by $T=\min \left(j \geq 0: A_{j} \in \mathcal{O}\right\}$. The expected value of $T$, given initial state $A_{0}=s_{i}$, is defined as

$$
m_{i, \mathcal{O}}=E\left(T \mid A_{0}=s_{i}\right)
$$

Definition 10 covers $|\mathcal{O}| \geq 1$ and thus includes first passage time into multiple optimal states. For simplicity we emphasize the one-state case $|\mathcal{O}|=1$ here, with $\mathcal{O}=$ $\left\{s^{*}\right\}=\left\{s_{k}\right\}$. First passage time now simplifies to $T=\min \left(j \geq 0: A_{j}=s_{k}\right\}$, and $m_{i, \mathcal{O}}$ simplifies to $m_{i, k}$.

Using conditional expectations, one obtains from Definition 10 the following wellknown result. 
Theorem 11 (Expected hitting time) Let $\mathcal{M}$ be a Markov chain with state space $\mathcal{S}=$ $\left\{s_{1}, \ldots, s_{k}\right\}$ and first passage time $T$ (into $s^{*}=s_{k}$ ). The expected hitting time $h$ is then

$$
h=\sum_{i=1}^{k} E\left(T \mid A_{0}=i\right) \operatorname{Pr}\left(A_{0}=i\right)=\sum_{i=1}^{k} m_{i} \pi_{i} .
$$

Expected hitting time can be used to analyze the expected time to reach an optimal state $s^{*} \in \mathcal{O}$ in an SLS model $(\mathcal{M}, \mathcal{O})$, and is often closely related to the observed mean run time for an SLS algorithm. For SLS, the hitting time $h$ is with respect to some state in $\mathcal{O}$ and depends on the algorithm's input parameters including the problem instance. Previously, we have studied hitting time $h\left(p_{N}\right)$, where $p_{N}$ is the noise probability [57]. By varying $p_{N}$, we have constructed expected hitting time curves that are analytical counterparts to experimental noise response curves [57]. These expected hitting time curves provide an analytical foundation for finding optimal noise level $p_{N}^{*}$. This noise level is optimal in the sense that it minimizes expected hitting time, or $p_{N}^{*}=\arg \min _{0 \leq p \leq 1} h(p)$.

\section{Measures of Utility and Gain}

Stochastic local search algorithms are based on evaluating the absolute goodness (utility) of an explanation and relative goodness (gain) of an explanation compared to explanations that are neighbors in the search space. Gain is, informally speaking, change in utility from one explanation $\boldsymbol{x}$ to another explanation $\boldsymbol{x}^{\prime}$. The explanation $\boldsymbol{x}^{\prime}$ may be derived from $\boldsymbol{x}$, for example by changing (flipping) one node's state.

Measures of utility and gain play essential roles in any local search algorithm, including SGS, and this section focuses on these matters. We first present measures of utility in Section 3.1, then measures of gain in Section 3.2. We highlight two different approaches, the multiplicative approach and the additive approach. While the multiplicative approach is natural, the additive approach is related to MAXSAT and turns out to give excellent performance in partly deterministic BNs. The basis for how the measures of gain are utilized in stochastic local search is described in Section 3.3.

Readers interested in our portfolio approach but not in our approach to handling zeros in BNs may want to skip this section.

\subsection{Measures of Utility}

A utility measure (or function) is also known as a cost function, an energy function, a fitness function, or an objective function. We introduce the notion of a utility measure $U(\boldsymbol{x})$, where $\boldsymbol{x}$ is an explanation in a $\mathrm{BN}$.

\subsubsection{Multiplicative Utility}

As one might expect, the definition of a joint probability from Eq. 1 is used as a utility measure in SLS. For purposes of notational convenience and uniform treatment in our SLS algorithms we also consider it a multiplicative utility $U_{M}$. 
Definition 12 (Multiplicative utility) Let $\boldsymbol{x}=(\boldsymbol{y}, \boldsymbol{e})$ be an explanation in a BN with $n$ nodes and evidence $\boldsymbol{e}$. The multiplicative utility is defined as

$$
U_{M}(\boldsymbol{x})=\operatorname{Pr}(\boldsymbol{x})=\prod_{i=1}^{n} \operatorname{Pr}\left(x_{i} \mid \pi\left(X_{i}\right)\right) .
$$

While multiplicative utility is a natural utility measure, it does have limitations, in particular in so-called partly deterministic BNs. These are BNs with many zeros in their CPTs and consequently many explanations $\boldsymbol{x}$ with $U_{M}(\boldsymbol{x})=\operatorname{Pr}(\boldsymbol{x})=0$.

To obtain further insight into partly deterministic BNs, suppose that zeros are assigned to CPT entries in an existing $\mathrm{BN}$ according to the following randomized algorithm. The algorithm is controlled by a probability parameter $\rho$, where $0 \leq \rho \leq 1$ (see [21] for details). With the exception of the last entry in a CPT column, $\rho$ is the probability that a CPT entry is set to zero. Specifically, $\rho$ controls the setting of zeros in the CPT entries of a BN node in the following manner. We assume a random number generator $\operatorname{RANDOM}(a, b)$ that generates a real number $r$, where $a \leq r \leq b$, uniformly at random. Let $V$ be an arbitrary non-root node in the $\mathrm{BN}$, and let $\boldsymbol{y}$ be an arbitrary instantiation of parent nodes $\boldsymbol{Y}=\Pi(V)$. The algorithm sequentially assigns probabilities to $V$ 's CPT entries, given $\boldsymbol{y}$, and distinguishes these two cases. Case 1 is for a state $v_{i} \in \Omega_{V}$ that is not the last state of $V$ : If $\operatorname{Random}(0,1)<\rho$ then put $\operatorname{Pr}\left(V=v_{i} \mid \boldsymbol{Y}=\boldsymbol{y}\right)=0$, else put $\operatorname{Pr}\left(V=v_{i} \mid \boldsymbol{Y}=\boldsymbol{y}\right)>0$. Case 2 is for the state $v_{|V|} \in \Omega_{V}$ that is the last state: Let $\operatorname{Pr}\left(V=v_{|V|} \mid \boldsymbol{Y}=\boldsymbol{y}\right)=$ $1-\sum_{i=1}^{|V|-1} \operatorname{Pr}\left(V=v_{i} \mid \boldsymbol{Y}=\boldsymbol{y}\right)$. The situation where $V$ is a root node in the $\mathrm{BN}$ is handled in a similar manner.

For the above randomized algorithm, the following theorem holds.

Theorem 13 Consider a BN $\beta$ with $n$ nodes, with $w=\Omega\left(V_{1}\right)=\cdots=\Omega\left(V_{n}\right)$, and where CPTs are set according to the above randomized algorithm. Let $\boldsymbol{x}$ be an explanation picked uniformly at random in $\beta$. Then

$$
\operatorname{Pr}\left(U_{M}(\boldsymbol{x})=0\right)=1-\left(1-\frac{w-1}{w} \times \rho\right)^{n} .
$$

This theorem is a slight variation on a result in [21]. Figure 1 illustrates (4), and clearly shows the rapid speed at which the probability $\operatorname{Pr}\left(U_{M}(\boldsymbol{x})=0\right)$ grows, for an explanation $\boldsymbol{x}$, with growing $\mathrm{BN}$ size $n$ and probability parameter $\rho$. The slowest growth for $\operatorname{Pr}\left(U_{M}(\boldsymbol{x})=0\right)$ in Fig. 1 is for $w=2$ and $\rho=0.1$, where for $n=10$ we have $\operatorname{Pr}\left(U_{M}(\boldsymbol{x})=0\right) \approx 0.4$. The fastest growth, on the other hand, takes place for $w=8$ and $\rho=0.9$, where already for $n=10$ we have $\operatorname{Pr}\left(U_{M}(\boldsymbol{x})=0\right) \approx 1.0$. In other words, almost all explanations picked uniformly at random have zero probability or $U_{M}(\boldsymbol{x})=0$. Since most application BNs of interest contain substantially more than $n=10$ nodes, the effect of zeros in CPTs is often much worse than indicated by these two examples.

As presented in Table 3, a large percentage of zeros can often be found in CPTs of application BNs. Theorem 13 suggests that in such BNs, if an explanation $\boldsymbol{x}$ is picked uniformly at random, $U_{M}(\boldsymbol{x})=\operatorname{Pr}(\boldsymbol{x})=0$ with high probability. Further, by the SAT reduction it is NP-hard to find an explanation $\boldsymbol{x}$ such that $\operatorname{Pr}(\boldsymbol{x})>0[72,80]$. The case $U_{M}(\boldsymbol{x})=\operatorname{Pr}(\boldsymbol{x})=0$ therefore presents a serious complication for the SLS approach to computing MPEs. With a large part of the search space of explanations equal to zero, there is often no gradient to hill-climb for an SLS algorithm using $U_{M}$. This 
BN node state space size $w=2$

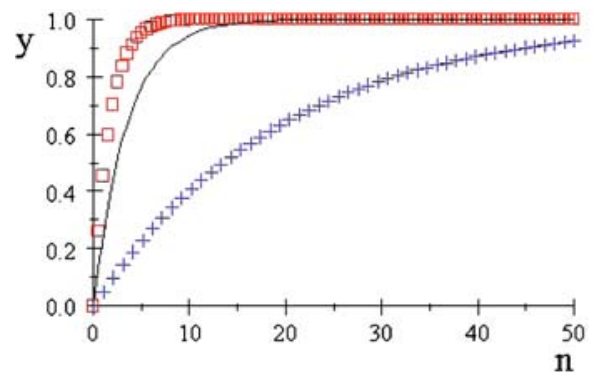

BN node state space size $w=8$

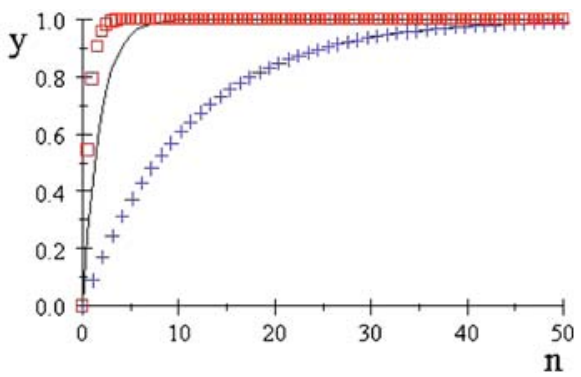

Fig. 1 The probability of a randomly picked BN explanation $\boldsymbol{x}$ being zero, or $y=\operatorname{Pr}\left(U_{M}(\boldsymbol{x})=0\right)$, as a function of varying BN size $n$ (along the $x$-axis), CPT entry zero probability $\rho$, and BN node state space size $w$. The curves are for varying $\rho$, with $\rho=0.1$ (curve indicated by blue crosses), $\rho=0.5$ (curve indicated by black line), and $\rho=0.9$ (curve indicated by red squares). The BN node state space size is different in these two panels. Left panel: State space size $w=2 ;$ Right panel: State space size $w=8$

limitation of the multiplicative measure leads us to the additive approach, which we discuss below. ${ }^{2}$

\subsubsection{Additive Utility}

Due to the limitations of multiplicative utility, we now consider a complementary utility measure, namely additive utility $U_{A}[54,60]$. This utility measure is based on the MAXSAT utility measure, which was used in the seminal GSAT family of algorithms [77, 78].

Definition 14 (Additive utility) Let $\boldsymbol{x}=(\boldsymbol{y}, \boldsymbol{e})$ be an explanation in a BN with $n$ nodes and evidence $\boldsymbol{e}$. The additive utility is defined as

$$
U_{A}(\boldsymbol{x})=\sum_{i=1}^{n} \operatorname{Pr}\left(x_{i} \mid \pi\left(X_{i}\right)\right) .
$$

Additive utility clearly avoids multiplicative utility's problem with zeros as discussed above in Section 3.1.1. The detailed investigation and use of the additive utility measure in $\mathrm{BN}$ inference is, to our knowledge, a novel aspect of our research as reported in this article.

We provide formal and experimental arguments in support of additive utility and gain in the following. First, as shown in Theorem 15 below, additive utility is a generalization of MAXSAT utility in the following sense. MAXSAT utility adds the number of satisfied CNF clauses [76,77], given a truth assignment to the logic variables. In a SAT-like BN, additive utility adds the probabilities of root and leaf nodes, given an instantiation of root nodes (which correspond to SAT variables) and

\footnotetext{
${ }^{2}$ In addition, this analysis underlines the importance of related work on handling determinism, for example by means of compilation into arithmetic circuits [4] or by encoding BNs into weighted MAXSAT problem instances and then apply weighted MAXSAT solvers [66, 74].
} 
clamping of the leaf nodes (which correspond to SAT clauses) to 1. Second, additive utility is an approximation to multiplicative utility. (Formal results concerning the relationship between additive gain and multiplicative gain are provided later in Section 3.) Third, it turns out that additive utility and gain give excellent empirical results in certain BNs as reported in Sections 6.2 and 6.1.

Theorem 15 (Generalization of MAXSAT) Let $\phi=(\boldsymbol{y}, \boldsymbol{q}, \boldsymbol{c})$ be a SAT problem instance with $|\boldsymbol{y}|=V,|\boldsymbol{c}|=C$, and $n=C+V$. Let $\beta=(\boldsymbol{X}, \boldsymbol{W}, \boldsymbol{P})$ be a Bayesian network that is $S A T$-equivalent to $\phi$ and with leaf nodes clamped, i.e. $\boldsymbol{e}=\left\{X_{1}=\right.$ $\left.1, \ldots, X_{C}=1\right\}$. Let $\boldsymbol{\tau}$ be a truth assignment to the logic variables $\boldsymbol{y}=\left\{y_{1}, \ldots, y_{V}\right\}$ in $\phi$ and let $\boldsymbol{x}=\left\{X_{C+1}=x_{c+1}, \ldots, X_{n}=x_{n}\right\}$ be an equivalent explanation over the $V$ root nodes in $\beta$. Then $U_{S}(\boldsymbol{\tau})=k$ if and only if ${ }^{3}$

$$
U_{A}(\boldsymbol{x})=k+\frac{V}{2}
$$

Proof Case $(\Leftarrow)$ : Consider a SAT-equivalent BN $\beta$ with an explanation $\boldsymbol{x}$, and suppose that $U_{A}(\boldsymbol{x})=k+\frac{V}{2}$. Now suppose that $\beta$ has $n$ nodes $\left\{X_{1}, \ldots, X_{n}\right\}$, and form

$$
U_{A}(\boldsymbol{x})=\sum_{i=1}^{n} \operatorname{Pr}\left(X_{i}=x_{i} \mid \pi\left(X_{i}\right)\right) .
$$

Since $\beta$ is SAT-equivalent to some formula $\phi, \beta$ is bipartite with $V$ root nodes (corresponding to variables in $\phi$ ) and $C=n-V$ leaf nodes (corresponding to clauses in $\phi)$. We decompose the additive utility $U_{A}(\boldsymbol{x})$ accordingly to

$$
U_{A}(\boldsymbol{x})=\sum_{i=1}^{C} \operatorname{Pr}\left(X_{i}=x_{i} \mid \pi\left(X_{i}\right)\right)+\sum_{i=C+1}^{n} \operatorname{Pr}\left(X_{i}=x_{i}\right),
$$

where $\left\{X_{C+1}, \ldots, X_{n}\right\}$ are root nodes and have no parents. By construction, the $V=$ $n-C$ root nodes are binary and each root node state has probability mass $\frac{1}{2}[8,72$, 80]. Since there are $V=n-C$ of them we obtain for the second term in (7)

$$
\sum_{i=C+1}^{n} \operatorname{Pr}\left(X_{i}=x_{i}\right)=\sum_{j=1}^{V} \operatorname{Pr}\left(X_{j+C}=x_{j+C}\right)=\frac{V}{2} .
$$

Clearly, (7) can be written as

$$
\sum_{i=1}^{C} \operatorname{Pr}\left(X_{i}=x_{i} \mid \pi\left(X_{i}\right)\right)=U_{A}(\boldsymbol{x})-\sum_{i=C+1}^{n} \operatorname{Pr}\left(X_{i}=x_{i}\right)=k
$$

where the last equality follows from $U_{A}(\boldsymbol{x})=k+\frac{V}{2}$ and (8). Now, in $\beta, C$ leaf nodes are being clamped as follows: $\boldsymbol{e}=\left\{X_{1}=1, \ldots, X_{C}=1\right\}$. Without loss of generality

${ }^{3}$ Recall that $U_{s}$ is MAXSAT utility; see Definition 5 for the formal definition. 
we assume a suitable ordering, and from (9) we know that for explanation $\boldsymbol{x}$ the leaf nodes in $\beta$ look like this:

$$
\begin{aligned}
\operatorname{Pr}\left(X_{1}=1 \mid \pi_{X_{1}}\right) & =1, \ldots, \operatorname{Pr}\left(X_{k}=1 \mid \pi_{X_{k}}\right)=1, \operatorname{Pr}\left(X_{k+1}=1 \mid \pi_{X_{k+1}}\right) \\
& =0, \ldots, \operatorname{Pr}\left(X_{C}=1 \mid \pi_{X_{C}}\right)=0 ;
\end{aligned}
$$

Due to the equivalence between explanations in $\beta$ and truth assignments in $\phi$, there is an assignment $\boldsymbol{\tau}$ for $\phi$ that is SAT-equivalent to explanation $\boldsymbol{x}$. And from the above it follows that exactly $k \leq C$ clauses are satisfied in $\phi$ under $\tau$, or $U_{S}(\tau)=k$. Case $(\Rightarrow)$ is similar, giving the desired result.

While the MAXSAT measure $U_{S}$ applies to the variables in a SAT formula, the additive measure $U_{A}$ applies to the full explanation in a BN, with BN nodes in a SATlike BN corresponding to both variables and clauses in the SAT-equivalent formula. More importantly, additive utility is not restricted to the logical setting as MAXSAT is. The additive measure $U_{A}$ can therefore be applied in all BNs, not only in SAT-like BNs.

Even though the $U_{A}$ measure clearly does not compute an explanation's probability, it is very useful for the special but important case of deterministic BN nodes. As already noted, surprisingly many BNs from applications have substantial deterministic fragments. Examples of such partly deterministic BNs are SAT-like BNs as discussed above (with or-nodes), error correction decoding BNs (with xornodes) from information theory [22, 49], and other BNs from applications-see Table 3.

\subsection{Measures of Gain}

For an explanation $\boldsymbol{x}^{\prime}$, a measure of gain, say $\Delta U$, simply measures change in utility $U$ compared to another explanation $\boldsymbol{x}$. When a node $X$ is flipped in an explanation $\boldsymbol{x}$, it has a localized impact in the $\mathrm{BN}$ at hand. The following definition introduces a vocabulary for such localized changes. To simplify the exposition, we often assume $\left|\Omega_{X}\right|=2$ in the remainder of this section; this can easily be generalized to $\left|\Omega_{X}\right| \geq 2$.

Definition 16 (Flipped node, flipped explanation) Consider an instantiated BN node $X_{i}=x_{i}$. If one puts $X_{i}=\bar{x}_{i}$, where $\bar{x}_{i}=0$ if $x_{i}=1$ and $\bar{x}_{i}=1$ if $x_{i}=0$, then the node is flipped (from $X_{i}=x_{i}$ ). An explanation $\boldsymbol{x}^{\prime}$ is flipped from another explanation $\boldsymbol{x}$ if at least one node $X_{i}$ has $X_{i}=x_{i}$ in $\boldsymbol{x}$ while $X_{i}=\bar{x}_{i}$ in $\boldsymbol{x}^{\prime}$.

We note that the terminology "flipping" does not imply that CPTs are being changed-we are not performing machine learning but rather search.

In the following definition, the notation $\boldsymbol{x}\left[X_{i}=x_{i}\right]$ means that the current state of the BN node $X_{i}$ is $x_{i}$ in the explanation $\boldsymbol{x}$; no changes are made. The notation $\boldsymbol{x}^{\prime}=\boldsymbol{x}\left[X_{i} \leftarrow \bar{x}_{i}\right]$, on the other hand, means that $X_{i}$ (which was assigned state $x_{i}$ in $\boldsymbol{x}$ ) is now, after the assignment $X_{i} \leftarrow \bar{x}_{i}$, assigned state $\bar{x}_{i}$ in $\boldsymbol{x}^{\prime}$. This is similar to assignment in programming languages.

Definition 17 (One-flipped) An explanation $\boldsymbol{x}^{\prime}=\left\{X_{m+1}=x_{m+1}, \ldots, X_{i}=\right.$ $\left.\bar{x}_{i}, \ldots, X_{n}=x_{n}\right\}$ is one-flipped from an explanation $\boldsymbol{x}=\left\{X_{m+1}=x_{m+1}, \ldots, X_{i}=\right.$ $\left.x_{i}, \ldots, X_{n}=x_{n}\right\}$ if exactly one node, here the $i$-th node, is flipped. For one-flipped 
explanations the notation $\boldsymbol{x}^{\prime}=\boldsymbol{x}\left[X_{i} \leftarrow \bar{x}_{i}\right]$, where $\boldsymbol{x}^{\prime}$ is the new explanation, is also used. A one-flipped parent instantiation, denoted $\bar{\pi}_{X}$, means that some node $Y$ $\in \Pi_{X}$ has its state flipped from $y$ to $\bar{y}$ in $X$ 's parent instantiation $\pi_{X}$.

At the core of SLS algorithms is an evaluation of whether the state of a node $X_{i}$ should be flipped from $x_{i}$ to $\bar{x}_{i}$, leading to a corresponding one-flipped change in explanation from $\boldsymbol{x}\left[X_{i}=x_{i}\right]$ to $\boldsymbol{x}\left[X_{i} \leftarrow \bar{x}_{i}\right]$. These evaluations are based on the concept of gain. We now discuss multiplicative gain, which is derived from multiplicative utility.

\subsubsection{Multiplicative Gain}

The multiplicative gain is obtained as follows. Let $\boldsymbol{x}$ be the explanation before flipping, $\boldsymbol{x}^{\prime}$ the explanation after flipping some number of nodes. A measure of the goodness of those flips is introduced in the following definition.

Definition 18 (Multiplicative gain, one-flip multiplicative gain) Let $\boldsymbol{x}$ and $\boldsymbol{x}^{\prime}$ be explanations, assume that $U_{M}(\boldsymbol{x})>0$, and suppose that we flip from $\boldsymbol{x}$ to $\boldsymbol{x}^{\prime}$. The multiplicative gain $\Delta U_{M}\left(\boldsymbol{x}, \boldsymbol{x}^{\prime}\right)$ is defined as

$$
\Delta U_{M}\left(\boldsymbol{x}, \boldsymbol{x}^{\prime}\right)=\frac{U_{M}\left(\boldsymbol{x}^{\prime}\right)}{U_{M}(\boldsymbol{x})} .
$$

Suppose that $x_{i, k} \in \Omega_{X_{i}}, x_{i, j} \in \Omega_{X_{i}}$, and $\boldsymbol{x}\left[X_{i}=x_{i, k}\right]$. The general case of one-flipping 'x's $X_{i}$ from $x_{i, k}$ to $x_{i, j}$, where $j \neq k$, is defined as

$$
\Delta U_{M}\left(\boldsymbol{x}, x_{i, j}\right)=\frac{U_{M}\left(\boldsymbol{x}\left[X_{i} \leftarrow x_{i, j}\right]\right)}{U_{M}\left(\boldsymbol{x}\left[X_{i}=x_{i, k}\right]\right)} .
$$

Definition 19 (Binary one-flip multiplicative gain) Assume that $X_{i}$ is binary, $\boldsymbol{x}\left[X_{i}=\right.$ $\left.x_{i}\right]$, and let $\boldsymbol{x}^{\prime}=\boldsymbol{x}\left[X_{i} \leftarrow \bar{x}_{i}\right]$. Binary one-flip multiplicative gain is then defined as

$$
\Delta U_{M}\left(\boldsymbol{x}, \bar{x}_{i}\right)=\frac{U_{M}\left(\boldsymbol{x}^{\prime}\right)}{U_{M}(\boldsymbol{x})}=\frac{U_{M}\left(\boldsymbol{x}\left[X_{i} \leftarrow \bar{x}_{i}\right]\right)}{U_{M}\left(\boldsymbol{x}\left[X_{i}=x_{i}\right]\right)} .
$$

We now show how multiplicative gain computation can be simplified compared to the above definitions.

Theorem 20 (One-flip multiplicative gain) Let $\boldsymbol{x}$ and $\boldsymbol{x}^{\prime}$ be explanations such that $\boldsymbol{x}^{\prime}=$ $\boldsymbol{x}\left[X_{i} \leftarrow \bar{x}_{i}\right]$. Further, let $\boldsymbol{C}=\Psi_{X_{i}}$, let $C_{j} \in \boldsymbol{C}$, and let $\pi\left(C_{j}\right) \subseteq \boldsymbol{x}$ (respectively $\bar{\pi}\left(C_{j}\right) \subseteq$ $\left.\boldsymbol{x}^{\prime}\right)$ be the instantiation of all parent nodes for children of $X_{i}$ before (respectively after) a one-flip of $X_{i}$. Suppose that $U_{M}(\boldsymbol{x})>0$. The one-flip multiplicative gain $\Delta U_{M}\left(\boldsymbol{x}, \bar{x}_{i}\right)$ is

$$
\Delta U_{M}\left(\boldsymbol{x}, \bar{x}_{i}\right)=\frac{\operatorname{Pr}\left(\bar{x}_{i} \mid \pi\left(X_{i}\right)\right) \times \prod_{C_{j} \in \boldsymbol{C}} \operatorname{Pr}\left(C_{j}=c_{j} \mid \bar{\pi}\left(C_{j}\right)\right)}{\operatorname{Pr}\left(x_{i} \mid \pi\left(X_{i}\right)\right) \times \prod_{C_{j} \in \boldsymbol{C}} \operatorname{Pr}\left(C_{j}=c_{j} \mid \pi\left(C_{j}\right)\right)},
$$

with $\operatorname{Pr}\left(x_{i} \mid \pi\left(X_{i}\right)\right) \times \prod_{C_{j} \in \boldsymbol{C}} \operatorname{Pr}\left(C_{j}=c_{j} \mid \pi\left(C_{j}\right)>0\right.$. 
Proof We start with the definitions of multiplicative gain and utility, obtaining

$$
\Delta U_{M}\left(\boldsymbol{x}, \bar{x}_{i}\right)=\frac{U_{M}\left(\boldsymbol{x}\left[X_{i} \leftarrow \bar{x}_{i}\right]\right)}{U_{M}(\boldsymbol{x})}=\frac{\operatorname{Pr}\left(\boldsymbol{x}\left[X_{i} \leftarrow \bar{x}_{i}\right]\right)}{\operatorname{Pr}(\boldsymbol{x})} .
$$

We now consider $X_{i}$ 's children $\boldsymbol{C}=\Psi_{X_{i}}$. Let $\boldsymbol{Y}$ be the BN nodes other than $X_{i}$ and $\boldsymbol{C}: \boldsymbol{Y}=\boldsymbol{V}-\left(\left\{X_{i}\right\} \cup \boldsymbol{C}\right)$, and rewrite Eq. 14 as

$\Delta U_{M}\left(\boldsymbol{x}, \bar{x}_{i}\right)=\frac{\operatorname{Pr}\left(X_{i}=\bar{x}_{i} \mid \pi\left(X_{i}\right)\right) \times \prod_{C_{j} \in \boldsymbol{C}} \operatorname{Pr}\left(C_{j}=c_{j} \mid \bar{\pi}\left(C_{j}\right)\right) \times \prod_{Y \in \boldsymbol{Y}} \operatorname{Pr}(Y=y \mid \pi(Y))}{\operatorname{Pr}\left(X_{i}=x_{i} \mid \pi\left(X_{i}\right)\right) \times \prod_{C_{j} \in \boldsymbol{C}} \operatorname{Pr}\left(C_{j}=c_{j} \mid \pi\left(C_{j}\right)\right) \times \prod_{Y \in \boldsymbol{Y}} \operatorname{Pr}(Y=y \mid \pi(Y))}$.

Since only one node $X_{i}$ is flipped (from $x_{i}$ to $\bar{x}_{i}$ ), we can exploit the Markov blanket locality of the BN and only need to consider the CPT in $X_{i}$ itself as well as CPTs for children $\boldsymbol{C}$, and obtain

$$
\Delta U_{M}\left(\boldsymbol{x}, \bar{x}_{i}\right)=\frac{\operatorname{Pr}\left(X_{i}=\bar{x}_{i} \mid \pi_{X_{i}}\right) \times \prod_{C_{j} \in \boldsymbol{C}} \operatorname{Pr}\left(C_{j}=c_{j} \mid \bar{\pi}\left(C_{j}\right)\right)}{\operatorname{Pr}\left(X_{i}=x_{i} \mid \pi_{X_{i}}\right) \times \prod_{C_{j} \in \boldsymbol{C}} \operatorname{Pr}\left(C_{j}=c_{j} \mid \pi\left(C_{j}\right)\right)} .
$$

This is exactly the multiplicative gain $\Delta U_{M}(\boldsymbol{x}, \bar{x})$ in Eq. 13. Obviously, since by assumption $U_{M}(\boldsymbol{x})>0$ it is clear that $\operatorname{Pr}\left(x_{i} \mid \pi\left(X_{i}\right)\right) \times \prod_{C_{j} \in \boldsymbol{C}} \operatorname{Pr}\left(C_{j}=c_{j} \mid \pi\left(C_{j}\right)\right)>0$ as well.

Note that the computation of $\operatorname{Pr}\left(\boldsymbol{x}^{\prime}\right)$ of a flipped explanation can be expressed as taking the probability of the old explanation, $\operatorname{Pr}(\boldsymbol{x})$, and multiplying it with the gain from Eq. 10, giving

$$
\operatorname{Pr}\left(\boldsymbol{x}^{\prime}\right)=\Delta U_{M}\left(\boldsymbol{x}, \boldsymbol{x}^{\prime}\right) \operatorname{Pr}(\boldsymbol{x})
$$

We typically restrict ourselves to the one-flipped case in this article, and consequently have $\boldsymbol{x}^{\prime}=\boldsymbol{x}\left[X_{i} \leftarrow \bar{x}_{i}\right]$ and thus obtain

$$
\begin{aligned}
\operatorname{Pr}\left(\boldsymbol{x}^{\prime}\right) & =\operatorname{Pr}\left(\boldsymbol{x}\left[X_{i} \leftarrow \bar{x}_{i}\right]\right) \\
& =\Delta U_{M}\left(\boldsymbol{x}, \bar{x}_{i}\right) \operatorname{Pr}(\boldsymbol{x}) \\
& =\frac{\operatorname{Pr}\left(X_{i}=\bar{x}_{i} \mid \pi_{X_{i}}\right) \times \prod_{C_{j} \in \boldsymbol{C}} \operatorname{Pr}\left(C_{j}=c_{j} \mid \bar{\pi}\left(C_{j}\right)\right)}{\operatorname{Pr}\left(X_{i}=x_{i} \mid \pi_{X_{i}}\right) \times \prod_{C_{j} \in \boldsymbol{C}} \operatorname{Pr}\left(C_{j}=c_{j} \mid \pi\left(C_{j}\right)\right)} \operatorname{Pr}(\boldsymbol{x}) .
\end{aligned}
$$

Especially when the number of nodes $n$ is large, using (16) iteratively rather than evaluating all of $\boldsymbol{x}^{\prime}$ to compute $\operatorname{Pr}\left(\boldsymbol{x}^{\prime}\right)$ provides substantial computational savings.

In the use of multiplicative gain in SGS and other SLS algorithms there is a slight but crucial complication in that multiplicative gain is not defined, according to Definition 18 , if the denominator $U_{M}(\boldsymbol{x})=0$. In practice, $U_{M}(\boldsymbol{x})=0$ is very common in partly deterministic BNs. We discuss how this can be handled in an adhoc manner in SGS in Section 4.1. However, this limitation of $U_{M}(\boldsymbol{x})$ also motivates our investigation of additive gain which we turn to now.

\subsubsection{Additive Gain}

The assumption $U_{M}(\boldsymbol{x})=\operatorname{Pr}(\boldsymbol{x})>0$ made for multiplicative gain in Eq. 10 is sometimes not realistic and leads us to introduce additive gain based upon additive utility. 
Definition 21 (Additive gain, one-flip additive gain) Let $\boldsymbol{x}$ and $\boldsymbol{x}^{\prime}$ be explanations. The additive gain $\Delta U_{A}\left(\boldsymbol{x}, \boldsymbol{x}^{\prime}\right)$ is defined as

$$
\Delta U_{A}\left(\boldsymbol{x}, \boldsymbol{x}^{\prime}\right)=U_{A}\left(\boldsymbol{x}^{\prime}\right)-U_{A}(\boldsymbol{x}) .
$$

Suppose that $x_{i, k} \in \Omega_{X_{i}}, x_{i, j} \in \Omega_{X_{i}}$, and $\boldsymbol{x}\left[X_{i}=x_{i, k}\right]$. The general case of one-flipping 'x's $X_{i}$ from $x_{i, k}$ to $x_{i, j}$, where $j \neq k$, is defined as

$$
\Delta U_{A}\left(\boldsymbol{x}, x_{i, j}\right)=U_{A}\left(\boldsymbol{x}\left[X_{i} \leftarrow x_{i, j}\right]\right)-U_{A}\left(\boldsymbol{x}\left[X_{i}=x_{i, k}\right]\right) .
$$

Definition 22 (Binary one-flip additive gain) Assume that $X_{i}$ is binary, $\boldsymbol{x}\left[X_{i}=x_{i}\right]$, and let $\boldsymbol{x}^{\prime}=\boldsymbol{x}\left[X_{i} \leftarrow \bar{x}_{i}\right]$. Binary one-flip additive gain is then defined as

$$
\Delta U_{A}\left(\boldsymbol{x}, \bar{x}_{i}\right)=U_{A}\left(\boldsymbol{x}^{\prime}\right)-U_{A}(\boldsymbol{x})=U_{A}\left(\boldsymbol{x}\left[X_{i} \leftarrow \bar{x}_{i}\right]\right)-U_{A}\left(\boldsymbol{x}\left[X_{i}=x_{i}\right]\right) .
$$

The additive gain is well-defined in cases where the multiplicative gain is not. As a consequence, we can in the SGS implementation of additive gain computation (see Fig. 4) handle the case $\operatorname{Pr}(\boldsymbol{x})=0$ without complicating the stochastic local search algorithm (see Fig. 3) or changing the CPT entries (see [66]) as has been done when using multiplicative gain. As we will see in experiments, additive gain proves useful as a complement to multiplicative gain.

Theorem 23 (One-flip additive gain) Let $\boldsymbol{x}$ and $\boldsymbol{x}^{\prime}$ be explanations such that $\boldsymbol{x}^{\prime}=$ $\boldsymbol{x}\left[X_{i} \leftarrow \bar{x}_{i}\right]$. Further, let $\boldsymbol{C}=\Psi_{X_{i}}$, let $C_{j} \in \boldsymbol{C}$, and let $\pi\left(C_{j}\right) \subseteq \boldsymbol{x}$ (respectively $\bar{\pi}\left(C_{j}\right) \subseteq$ $\left.\boldsymbol{x}^{\prime}\right)$ be the instantiation of all parent nodes for children of $X_{i}$ before (respectively after) a one-flip of $X_{i}$. The one-flip additive gain $\Delta U_{A}\left(\boldsymbol{x}, \bar{x}_{i}\right)$ is then

$$
\begin{aligned}
\Delta U_{A}\left(\boldsymbol{x}, \bar{x}_{i}\right)=\operatorname{Pr}\left(\bar{x}_{i} \mid \pi_{X_{i}}\right) & +\sum_{C_{j} \in \boldsymbol{C}} \operatorname{Pr}\left(C_{j}=c_{j} \mid \bar{\pi}\left(C_{j}\right)\right)-\operatorname{Pr}\left(x_{i} \mid \pi_{X_{i}}\right) \\
& -\sum_{C_{j} \in \boldsymbol{C}} \operatorname{Pr}\left(C_{j}=c_{j} \mid \pi\left(C_{j}\right)\right) .
\end{aligned}
$$

Proof The one-flip additive gain can be derived in a manner similar to the proof of one-flip multiplicative gain $\Delta U_{M}(\boldsymbol{x}, \bar{x})$ in Theorem 20 .

\subsubsection{Additive Gain and Multiplicative Gain}

We have several arguments for why the use of additive gain may be productive. One reason, which is stated formally in Theorem 27 below, relates additive gain and multiplicative gain. In order to improve readability, we introduce in Definitions 24 and 25 alternative ways of expressing additive and multiplicative gains when flipping a node $X_{i}$ with children $\boldsymbol{C}$.

Definition 24 (Simple additive gain notation) Let $\alpha=|\boldsymbol{C}|+1$ and put in Theorem 23: $p_{1}=\operatorname{Pr}\left(\bar{x}_{k} \mid \pi_{X_{k}}\right), \sum_{i=2}^{\alpha} p_{i}=\sum_{C_{j} \in \boldsymbol{C}} \operatorname{Pr}\left(C_{j}=c_{j} \mid \bar{\pi}\left(C_{j}\right)\right), q_{1}=\operatorname{Pr}\left(x_{k} \mid \pi_{X_{k}}\right)$, and $\sum_{i=2}^{\alpha} q_{i}=\sum_{C_{j} \in C} \operatorname{Pr}\left(C_{j}=c_{j} \mid \pi\left(C_{j}\right)\right)$. This gives the following simplified notation for additive gain:

$$
\Delta U_{A}\left(\boldsymbol{x}, \bar{x}_{k}\right)=\sum_{i=1}^{\alpha} p_{i}-\sum_{i=1}^{\alpha} q_{i} .
$$


Definition 25 (Simple multiplicative gain notation) Let $|\boldsymbol{C}|=\alpha+1$ and put in Theorem 20: $p_{1}=\operatorname{Pr}\left(\bar{x}_{k} \mid \pi_{X_{k}}\right), \quad \prod_{i=2}^{\alpha} p_{i}=\prod_{C_{j} \in C} \operatorname{Pr}\left(C_{j}=c_{j} \mid \bar{\pi}\left(C_{j}\right)\right), \quad q_{1}=\operatorname{Pr}\left(x_{k} \mid\right.$ $\pi_{X_{k}}$ ), and $\prod_{i=2}^{\alpha} q_{i}=\prod_{C_{j} \in C} \operatorname{Pr}\left(C_{j}=c_{j} \mid \pi\left(C_{j}\right)\right)$. This gives the following simplified notation for multiplicative gain:

$$
\Delta U_{M}\left(\boldsymbol{x}, \bar{x}_{k}\right)=\prod_{i=1}^{\alpha} \frac{p_{i}}{q_{i}},
$$

again under the assumption $\prod_{i=1}^{\alpha} q_{i}>0$.

The following fact follows easily from the definitions of $\Delta U_{A}$ and $\Delta U_{M}$.

Proposition 26 Let $\boldsymbol{x}$ and $\boldsymbol{x}^{\prime}$ be explanations and suppose that $\Delta U_{M}(\boldsymbol{x})>0$. $\Delta U_{A}\left(\boldsymbol{x}, \boldsymbol{x}^{\prime}\right)=0$ if and only if $\Delta U_{M}\left(\boldsymbol{x}, \boldsymbol{x}^{\prime}\right)=1$.

We now turn to the more general case where $\Delta U_{A}\left(\boldsymbol{x}, \boldsymbol{x}^{\prime}\right) \neq 0$ and $\Delta U_{M}\left(\boldsymbol{x}, \boldsymbol{x}^{\prime}\right) \neq 1$.

Theorem 27 Consider the definitions of additive gain $\Delta U_{A}\left(\boldsymbol{x}, \bar{x}_{k}\right)=\sum_{i=1}^{\alpha} p_{i}-$ $\sum_{i=1}^{\alpha} q_{i}$ and multiplicative gain $\Delta U_{M}\left(\boldsymbol{x}, \bar{x}_{k}\right)=\prod_{i=1}^{\alpha} \frac{p_{i}}{q_{i}}$ from Definitions 24 and 25 respectively. Suppose that there exists a permutation $\sigma$ of $\left\{q_{1}, \ldots, q_{\alpha}\right\}$, denoted $\left\{q_{\sigma(1)}, \ldots, q_{\sigma(\alpha)}\right\}$, and a permutation $\rho$ of $\left\{p_{1}, \ldots, p_{\alpha}\right\}$, denoted $\left\{p_{\rho(1)}, \ldots, p_{\rho(\alpha)}\right\}$, such that

$$
p_{\rho(1)}>q_{\sigma(1)}, \ldots, p_{\rho(\kappa)}>q_{\sigma(\kappa)}, p_{\rho(\kappa+1)}=q_{\sigma(\kappa+1)}, \ldots, p_{\rho(\alpha)}=q_{\sigma(\alpha)}
$$

for some $1 \leq \kappa \leq \alpha$. If $(21)$ is true and $\Delta U_{A}\left(\boldsymbol{x}, \bar{x}_{k}\right)>0$ then $\Delta U_{M}\left(\boldsymbol{x}, \bar{x}_{k}\right)>1$.

Proof Since, by assumption, $\Delta U_{A}\left(\boldsymbol{x}, \boldsymbol{x}^{\prime}\right)=\sum_{i=1}^{\alpha} p_{i}-\sum_{i=1}^{\alpha} q_{i}>0$, we must have $\kappa \geq$ 1 in (21). For any $1 \leq j \leq \kappa$, it is the case that $p_{\rho(j)}>q_{\sigma(j)}$ or in other words $\frac{p_{\rho(j)}}{q_{\sigma(j)}}>1$, giving also

$$
\prod_{j=1}^{\kappa} \frac{p_{\rho(j)}}{q_{\sigma(j)}}>1 .
$$

For the remaining elements in (21) we have $p_{\rho(\kappa+1)}=q_{\sigma(\kappa+1)}, \ldots, p_{\rho(\alpha)}=q_{\sigma(\alpha)}$ and thus

$$
\prod_{j=\kappa+1}^{\alpha} \frac{p_{\rho(j)}}{q_{\sigma(j)}}=1 .
$$

Going back to the definition (20) as well as (22) and (23) gives

$$
\Delta U_{M}\left(\boldsymbol{x}, \bar{x}_{k}\right)=\prod_{i=1}^{\alpha} \frac{p_{i}}{q_{i}}=\prod_{j=1}^{\kappa} \frac{p_{\rho(j)}}{q_{\sigma(j)}} \prod_{j=\kappa+1}^{\alpha} \frac{p_{\rho(j)}}{q_{\sigma(j)}}>1
$$

as desired.

Theorem 27 justifies the use of $\Delta U_{A}$ in MPE local search algorithms as follows: When flipping from explanation $\boldsymbol{x}$ to explanation $\boldsymbol{x}^{\prime}$-and if $\Delta U_{A}\left(\boldsymbol{x}, \boldsymbol{x}^{\prime}\right)>0$ as well 
as condition (21) holds during local search-one is also making progress according to $\Delta U_{M}$.

Despite the result above, we emphasize that a positive additive gain $\Delta U_{A}$ does not always imply an increase in an explanation's probability or multiplicative gain $\Delta U_{M}$. This is stated formally in the following result.

Proposition 28 Let $\boldsymbol{x}$ be an explanation, and $\boldsymbol{x}^{\prime}$ be one-flipped from $\boldsymbol{x}$. It is not always the case that if $\Delta U_{A}\left(\boldsymbol{x}, \boldsymbol{x}^{\prime}\right)>0$ then $\Delta U_{M}\left(\boldsymbol{x}, \boldsymbol{x}^{\prime}\right)>1$.

Proof A counter-example in a BN with two nodes $X_{1}$ and $X_{2}$ is provided. The nodes both have state space $\{0,1\}$. Node $X_{1}$ is $X_{2}$ 's parent. Let the CPTs be $\left\{\operatorname{Pr}\left(X_{1}=0\right)=0.2, \operatorname{Pr}\left(X_{1}=1\right)=0.8, \operatorname{Pr}\left(X_{2}=0 \mid X_{1}=0\right)=0.4\right.$, $\left.\operatorname{Pr}\left(X_{2}=1 \mid X_{1}=0\right)=0.6, \operatorname{Pr}\left(X_{2}=0 \mid X_{1}=1\right)=0.05, \operatorname{Pr}\left(X_{2}=1 \mid X_{1}=1\right)=0.95\right\}$. Now consider $\boldsymbol{x}=\left\{X_{1}=0, X_{2}=0\right\}$ and $\boldsymbol{x}^{\prime}=\left\{X_{1}=1, X_{2}=0\right\}$. In this case we have $\Delta U_{A}\left(\boldsymbol{x}, \boldsymbol{x}^{\prime}\right)=U_{A}\left(\boldsymbol{x}^{\prime}\right)-U_{A}(\boldsymbol{x})=(0.8+0.05)-(0.2+0.4)=0.25>0$ while $\Delta U_{M}\left(\boldsymbol{x}, \boldsymbol{x}^{\prime}\right)=U_{M}\left(\boldsymbol{x}^{\prime}\right) / U_{M}(\boldsymbol{x})=(0.8 \times 0.05) /(0.2 \times 0.4)=0.5 \ngtr 1$.

\subsubsection{Additive Gain and GSAT Gain}

The reasoning behind the GSAT gain [77, 78] and the additive gain shown in Eq. 19 is similar. Reflecting this, we introduce the following two definitions based on previous research $[77,78]$.

Definition 29 (GSAT gain) Let $\tau$ and $\tau^{\prime}$ be truth assignments. Then GSAT gain $\Delta U_{S}\left(\boldsymbol{\tau}, \boldsymbol{\tau}^{\prime}\right)$ is defined as

$$
\Delta U_{S}\left(\boldsymbol{\tau}, \boldsymbol{\tau}^{\prime}\right)=U_{S}\left(\boldsymbol{\tau}^{\prime}\right)-U_{S}(\boldsymbol{\tau})
$$

The notation $\Delta U_{S}\left(\tau, \tau^{\prime}\right)$ is used for GSAT gain over truth assignments $\tau$ and $\tau^{\prime}$ for SAT formulas, similar to additive gain $\Delta U_{A}\left(\boldsymbol{x}, \boldsymbol{x}^{\prime}\right)$ for explanations $\boldsymbol{x}$ and $\boldsymbol{x}^{\prime}$ in BNs as introduced in Definition 21.

Definition 30 (One-flip GSAT gain) Let $\tau$ be a truth assignment to the variables $\boldsymbol{y}$ in a SAT instance $\phi=(\boldsymbol{y}, \boldsymbol{q}, \boldsymbol{c})$. Suppose that $\boldsymbol{\tau}$ is changed to $\overline{\boldsymbol{\tau}}$ by flipping one variable $y \in \boldsymbol{y}$ from $\tau(y)$ to $\bar{\tau}(y)$. Let $\operatorname{MakeCount}(\boldsymbol{\tau}, \bar{y})$ be the number of clauses satisfied (or made 1$)$ by the flip of $y$. Let BREAKCOUNT $(\boldsymbol{\tau}, \bar{y})$ be the number of clauses unsatisfied (or made 0 ) by the flip of $y$. One-flip GSAT gain is defined as

$$
\Delta U_{S}(\boldsymbol{\tau}, \bar{y})=\operatorname{MaKeCOUNT}(\boldsymbol{\tau}, \bar{y})-\operatorname{Break} \operatorname{Count}(\boldsymbol{\tau}, \bar{y}) .
$$

In a manner similar to GSAT gain's success in SAT, our additive gain turns out to be powerful on deterministic BN nodes, which occur in application BNs, as well as in SAT-like BNs which encode satisfiability instances [8, 72]. In fact, GSAT gain is a special case of additive gain as expressed in the following result.

Theorem 31 Let $B N \beta=(\boldsymbol{Y}, \boldsymbol{W}, \boldsymbol{P})$ and suppose that $S A T$ instance $\phi=(\boldsymbol{y}, \boldsymbol{q}, \boldsymbol{c})$ is $S A T$-equivalent to $\beta$. Further, let two explanations $\boldsymbol{x}_{1}$ and $\boldsymbol{x}_{2}$ in $\beta$ be $S A T$ equivalent to two truth assignments $\boldsymbol{\tau}_{1}$ and $\tau_{2}$, respectively, for $\phi$. Then $\Delta U_{A}\left(\boldsymbol{x}_{1}, \boldsymbol{x}_{2}\right)=$ $\Delta U_{S}\left(\boldsymbol{\tau}_{1}, \boldsymbol{\tau}_{2}\right)$. 
Proof Applying Theorem 15 to both $\boldsymbol{x}_{1}$ and $\boldsymbol{x}_{2}$ gives

$$
\begin{aligned}
& U_{A}\left(\boldsymbol{x}_{1}\right)=U_{S}\left(\boldsymbol{\tau}_{1}\right)+\frac{V}{2} \\
& U_{A}\left(\boldsymbol{x}_{2}\right)=U_{S}\left(\boldsymbol{\tau}_{2}\right)+\frac{V}{2},
\end{aligned}
$$

and when (24) is subtracted from (25) we obtain

$$
U_{A}\left(\boldsymbol{x}_{2}\right)-U_{A}\left(\boldsymbol{x}_{1}\right)=U_{S}\left(\boldsymbol{\tau}_{2}\right)-U_{S}\left(\boldsymbol{\tau}_{1}\right) .
$$

Simply substituting the definitions of $\Delta U_{A}\left(\boldsymbol{x}_{1}, \boldsymbol{x}_{2}\right)$ (Definition 21) and $\Delta U_{S}\left(\boldsymbol{\tau}_{1}, \boldsymbol{\tau}_{2}\right)$ (Definition 29) into (26) gives the desired result.

The following result follows easily from Theorem 31 .

Corollary 32 Let $\Delta U_{A}\left(\boldsymbol{x}_{1}, \bar{x}_{i}\right)$ be the additive gain obtained by flipping a root node $X_{i} \in \boldsymbol{X}$ in a SAT-like $B N \beta=(\boldsymbol{Y}, \boldsymbol{W}, \boldsymbol{P})$. Assume that the $S A T$ instance $\phi=(\boldsymbol{y}, \boldsymbol{q}, \boldsymbol{c})$ is $S A T$-equivalent to $\beta$ and that $\boldsymbol{\tau}_{1}$ is equivalent to $\boldsymbol{x}_{1}$. Further, let $\Delta U_{S}\left(\boldsymbol{\tau}_{1}, \bar{y}_{i}\right)$ be the GSAT gain obtained, under $\boldsymbol{\tau}_{1}$, by flipping the variable $y_{i} \in \boldsymbol{y}$ corresponding to the root node $X_{i} \in \boldsymbol{X}$. It is then the case that $\Delta U_{A}\left(\boldsymbol{x}_{1}, \bar{x}_{i}\right)=\Delta U_{S}\left(\boldsymbol{\tau}_{1}, \bar{y}_{i}\right)$.

Proof Put $\boldsymbol{x}_{2} \leftarrow \boldsymbol{x}_{1}\left[X_{i} \leftarrow \bar{x}_{i}\right]$ and let $\boldsymbol{\tau}_{2} \leftarrow \overline{\boldsymbol{\tau}}\left(\phi, y_{i}, \boldsymbol{\tau}_{1}\right.$ ) (copying $\boldsymbol{\tau}_{1}$ into $\boldsymbol{\tau}_{2}$ but exchanging $\boldsymbol{\tau}\left(y_{i}\right)$ with $\left.\overline{\boldsymbol{\tau}}\left(y_{i}\right)\right)$. Observe that node $X_{i}$ corresponds to the variable $y_{i}$. Clearly, the constructed truth assignment $\boldsymbol{\tau}_{2}$ is equivalent to the constructed explanation $\boldsymbol{x}_{2}$, thus Theorem 31 applies and the result follows.

\subsection{Criteria of Choice}

In addition to measures of utility and gain, SLS algorithms including SGS need criteria of choice. These criteria determine how an SLS algorithm decides, based on its gain computations, which BN node(s) to flip next. Gains in a neighborhood around the current explanation $\boldsymbol{x}$ are computed and placed in a candidate array (or set), defined as follows.

Definition 33 (Candidate array) Let $\boldsymbol{x}$ be an explanation and let $g_{i, j}=\Delta U\left(\boldsymbol{x}, x_{i, j}\right)$ be the one-flip gain obtained by flipping explanation $\boldsymbol{x}$ 's $i$-th node $X_{i}$ from its $k$ th state $k \in\left\{1, \ldots,\left|\Omega_{X_{i}}\right|\right\}$ to its $j$-th state, where $j \in\left\{1, \ldots,\left|\Omega_{X_{i}}\right|\right\}-\{k\}$. The set of three-tuples

$$
\begin{aligned}
\boldsymbol{A}=\{ & \left(X_{i}, x_{i, j}, g_{i, j}\right) \mid X_{i} \text { is the } i \text {-th BN node, } x_{i, j} \text { is } X_{i} \text { 's } j \text {-th state, } \\
& \text { and } \left.g_{i, j} \text { is the one-flip gain }\right\}
\end{aligned}
$$

is denoted the candidate array.

At present, $g_{i, j}=\Delta U\left(\boldsymbol{x}, x_{i, j}\right)$ is either multiplicative gain $\Delta U_{M}\left(\boldsymbol{x}, x_{i, j}\right)$ (11) or additive gain $\Delta U_{A}\left(\boldsymbol{x}, x_{i, j}\right)(18)$. The candidate array describes explanation $\boldsymbol{x}$ 's local neighborhood and is used to determine-using a criterion of choice-which node to actually flip in $\boldsymbol{x}$. We have investigated two types of criteria of choice, the greedy 
criterion $C_{G}$ and the noisy criterion $C_{N}$. Both criteria rely on the candidate array $\boldsymbol{A}$ and a tuple of gains in $\boldsymbol{A}$, formally $\boldsymbol{G}=\left(g_{1}, \ldots, g_{m}\right){ }^{4}$

Definition 34 (Greedy criterion $C_{G}$ ) The greedy (or maximizing) criterion $C_{G}(\boldsymbol{A})$ is defined as picking a tuple with gain $g_{\max }$ such that $g_{\max } \geq g_{j}$ for all $1 \leq j \leq m$. Among $k$ such maximal tuples, $C_{G}(\boldsymbol{A})$ picks the $i$-th tuple with probability $p_{i}=1 / k$ for $1 \leq$ $i \leq k$; all remaining tuples are picked with probability $p_{i}=0$ for $k+1 \leq i \leq m$.

Definition 35 (Noisy criterion $C_{N}$ ) The noisy (or stochastic) criterion $C_{N}(\boldsymbol{A})$ is defined as picking the $i$-th tuple with gain $g_{i}$, for $1 \leq i \leq m$, according to the probability distribution

$$
p_{i}=g_{i} / \sum_{j=1}^{m} g_{j} .
$$

It is easy to see that $\sum_{i=1}^{m} p_{i}=1$ in Definition 34 and in Definition 35, thus these are both valid probability distributions.

In SGS, the maximizing criterion in Definition 34 chooses greedily from the candidate array $\boldsymbol{A}$ and yields classical steepest-ascent hill-climbing. The stochastic criterion in Definition 35 yields probabilistic hill-climbing, since it probabilistically decides which node and state to flip to, using gain information in the candidate array $\boldsymbol{A}$. This stochastic criterion is closely related to the approach used in the stochastic simulation algorithm [71] as well as to noise strategies such as "random walk" used in stochastic local search for SAT [77].

A measure of gain, then, defines how to measure progress for candidate local search steps. A criterion of choice, on the other hand, determines how to choose between the different candidate local search steps, based on the candidate gains, using a maximizing criterion or a stochastic criterion.

\section{Stochastic Local Search Algorithms}

Stochastic greedy search (SGS) is a local search approach augmented with stochastic initialization and noise steps. This section presents two variants of SGS, in order of increasing complexity and power. Section 4.1 presents simple SGS (or SimPLESGS), while Section 4.2 presents operator-based SGS (or OperatorSGS). Among these, SIMPLESGS is most similar to other SLS algorithms, and acts as a stepping stone to reach OPERATORSGS, our portfolio-based main contribution.

\subsection{Simple Stochastic Greedy Search}

SIMPLESGS, which is presented in Fig. 2, starts from an explanation $\boldsymbol{x}$ as constructed by an initialization algorithm INITIALIZE and performs one-flip local changes to $\boldsymbol{x}$ in

\footnotetext{
${ }^{4}$ For the binary case and when there is no evidence, $m=n$ since an explanation in a BN with $n$ nodes has $n$ possible one-flip gains.
} 


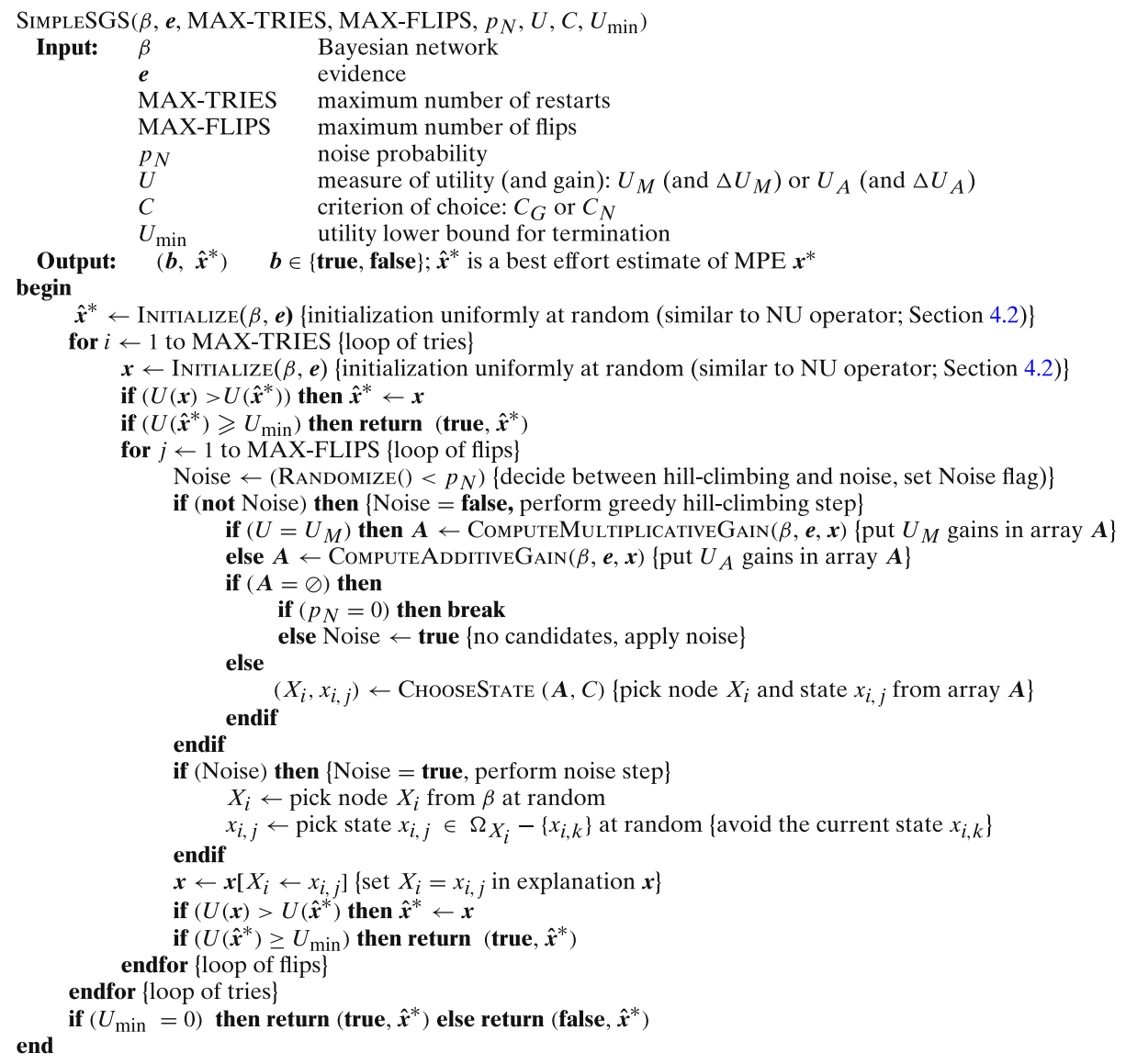

Fig. 2 The simple stochastic greedy search algorithm SimpLESGS. The algorithm repeatedly interleaves noise and greedy hill-climbing steps; hill-climbing is done by calling either ComPUTEMultiplicativeGain or ComputeAdditiveGain. These functions return a candidate array $\boldsymbol{A}$ which contains candidates for the next hill-climbing step. A candidate is a tuple $\left(X_{i}, x_{i, j}, g_{i, j}\right)$, where $X_{i}$ is the BN node potentially being flipped, $x_{i, j}$ is a state of that node, and $g_{i, j}$ is the gain obtained by flipping $X_{i}$ to $x_{i, j}$. The ChooseState function applies the criterion of choice $C$ to pick a node $X_{i}$ and state $x_{i, j}$, which is then subsequently flipped to

order to improve the utility $U(\boldsymbol{x})$. The input parameter $U$ controls which measure of utility and gain is used, and is currently either $U_{M}$ or $U_{A}$. The algorithm is related to the GSAT and WALKSAT algorithms [77, 78] as well as other SLS algorithms as discussed in Section 7: There is an outer loop for tries (or restarts), and an inner loop for flips (or stochastic local search steps). The parameter MAX-FLIPS limits how many flips are done before a restart, while the number of tries is upper bounded by MAX-TRIES. ${ }^{5}$ The parameter $p_{N}$ controls the noise level. Applying

\footnotetext{
${ }^{5}$ An alternative to the use of MAX-TRIES, not further pursued in this article, would be to use an upper bound on computation time in wall-clock time. Further discussion of termination criteria can be found in Section 5.1 .
} 
noise amounts to taking a random step with probability $p_{N}$, and taking a greedy step using the chosen measure of gain with probability $1-p_{N}$. This is similar to WALKSAT with random noise [77]. SIMPLESGS terminates when an explanation $\hat{\boldsymbol{x}}^{*}$ is found such that $U\left(\hat{\boldsymbol{x}}^{*}\right) \geq U_{\min }$. Other termination criteria can easily be incorporated into SIMPLESGS. In particular, we have implemented the option of terminating after executing SimpleSGS for a certain time period, even though this is not shown in Fig. 2.

The SimpleSGS algorithm returns a two-valued tuple $\left(\boldsymbol{b}, \hat{\boldsymbol{x}}^{*}\right)$. The first value $\boldsymbol{b}$ is a Boolean value signifying whether search was successful or not; the second value $\hat{\boldsymbol{x}}^{*}$ is the explanation with the highest utility score. The input value $p_{N}=0$ means that no noise should be applied; in this case SIMPLESGS returns when reaching a local maximum. With $U_{\min }>0$, SIMPLESGS is successful if utility $U_{\min }$ or better is attained. SimpleSGS uses gain computation algorithms ComputeMultiplicativeGain and ComputeAdditiveGain, see Figs. 3 and 4 respectively, to evaluate candidate tuples and construct the candidate array $\boldsymbol{A}$. Which gain computation is used depends on the value of the input parameter $U$. Both gain computation algorithms iterate over

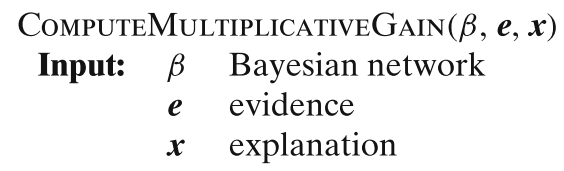

Output: $\boldsymbol{A}$ candidate array of gains computed using multiplicative gain begin

$\boldsymbol{X} \leftarrow$ nodes in $\boldsymbol{Y}$, where $\beta=(\boldsymbol{Y}, \boldsymbol{W}, \boldsymbol{P})$, that are not in $\boldsymbol{e}$ $\operatorname{InIT}(\boldsymbol{A}, 1)$ for multiplicative gain initialize candidate array $\boldsymbol{A}$ with 1s

for each non-evidence node $X_{i} \in \boldsymbol{X}$

$o \leftarrow x_{i}$ \{remember original state $x_{i}$ \}

Old $\leftarrow$ NodeChildrenProbability $\left(\beta, X_{i}, \boldsymbol{x}, U_{M}\right)$

for each state $s \in \Omega_{X_{i}}-\left\{x_{i}\right\}$ try all states except state $\left.x_{i}\right\}$

$\boldsymbol{x} \leftarrow \boldsymbol{x}\left[X_{i} \leftarrow s\right]$ try state $s$ in $i$-th node $X_{i}$ \}

New $\leftarrow$ NodeChildrenProbability $\left(\beta, X_{i}, \boldsymbol{x}, U_{M}\right)$

if $($ Old $=0)$ then

if $(\mathrm{New}=0)$ then $g \leftarrow 1$

else

Force $\left(\boldsymbol{A}, X_{i}, s\right)\{$ “force" state $s$ into candidate array $\boldsymbol{A}\}$ return $A$

endif

endif

$g \leftarrow$ New / Old

endfor

$\operatorname{AdD}\left(\boldsymbol{A}, X_{i}, s, g\right)\left\{\right.$ add tuple $\left(X_{i}, s, g\right)$ to candidate array $\left.\boldsymbol{A}\right\}$

endfor

$\boldsymbol{x} \leftarrow \boldsymbol{x}\left[X_{i} \leftarrow o\right]$ reset state of $X_{i}$ to original value $\left.o\right\}$

return $A$

end

Fig. 3 This algorithm, which implements the multiplicative measure of gain $\Delta U_{M}$, is called by SGS and is used to fill in the candidate array $\boldsymbol{A}$ with gains for neighbors of the current explanation $\boldsymbol{x}$ 


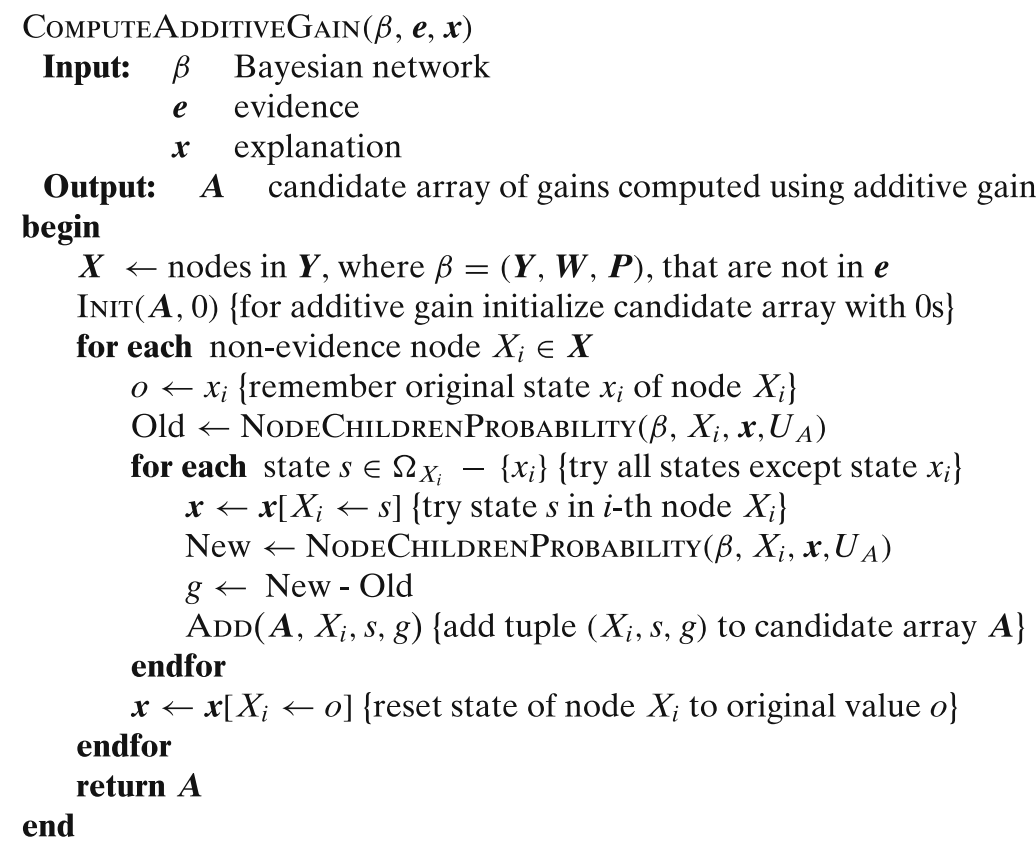

Fig. 4 This algorithm, which implements the additive measure of gain $\Delta U_{A}$, is used by SGS to fill in the candidate array $\boldsymbol{A}$ with gains for neighbors of the current explanation $\boldsymbol{x}$

all non-evidence nodes and over all states of each node $X_{i}$, excluding a node's current state $x_{i, k}$. Within the inner-most loops there are calls to NodeChILdRenProbability, which are used to compute the gain $g$ associated with flipping node $X_{i}$ 's state in explanation $\boldsymbol{x}$.

We now discuss how the ComputeMultiplicativeGain and ComputeAdditiveGAIN algorithms implement multiplicative gain $\Delta U_{M}$ and additive gain $\Delta U_{A}$ respectively. In the implementation of the multiplicative measure as shown in ComPuteMultiplicativeGain in Fig. 3, the assignment to Old is the denominator in multiplicative gain, while the assignment to New is multiplicative gain's numerator. Recall from Definition 18 and Theorem 20 that multiplicative gain is not defined if the denominator is zero. Consequently, there is a test for zero in ComputeMultiPLicAtiveGain, and the following action is taken: If Old (denominator in Eq. 12) and New (numerator in Eq. 12) are both zero, we define multiplicative gain $g$ to be one: $g \leftarrow 1$. If Old is zero but New is non-zero, we greedily pick the new state $s$ in the statement Force $\left(\boldsymbol{A}, X_{i}, s\right)$, and then return. So in this case where Old (denominator) is zero but New (numerator) is non-zero, the algorithm is more greedy than in the remaining cases, since the first state in the first node where a flip leads from a zero to a non-zero probability is picked as a candidate for flipping, by using ForCE.

Both ComputeMultiplicativeGain and ComputeAdditiveGain return a filledin candidate array $\boldsymbol{A}$ to SimpleSGS. After computation of $\boldsymbol{A}$, SimpleSGS calls the ChooseState function. ChooseState applies the criterion of choice and picks which state $x_{i, j}$ to flip to. Then, after a possible noise step, the utility measure $U(\boldsymbol{x})$ 
is applied. Using either multiplicative utility $U_{M}$ or additive utility $U_{A}$, the better explanation of $\boldsymbol{x}$ and $\hat{\boldsymbol{x}}^{*}$ is kept. The algorithm then terminates or iterates.

SIMPLESGS has been very successful in certain BNs, as is demonstrated in Section 6.1. It turned out that the algorithm also has some limitations, including the following. First, SimPLESGS does not accommodate an arbitrary number of different initialization and search operators in the same invocation of the algorithm. Second, the measures of utility and gain are coupled through the input parameter $U$, and it is not possible to apply different measures of gain in the same invocation of the algorithm. Addressing these limitations is crucial to obtaining strong performance. We now discuss how they are addressed in the operator-based variant of SGS.

\subsection{Operator-Based Stochastic Greedy Search}

The operator-based variant of SGS, denoted OpERATORSGS, is presented in Fig. 5. OpERATORSGS retains the structure of SimpleSGS, with two nested loops for tries and flips. What is novel in OperatorSGS is how the algorithm chooses from two sets of operators or heuristics when an initialization or search step is performed. This operator-based approach gives better flexibility and extensibility, resulting in substantially better performance on a range of different BNs compared to

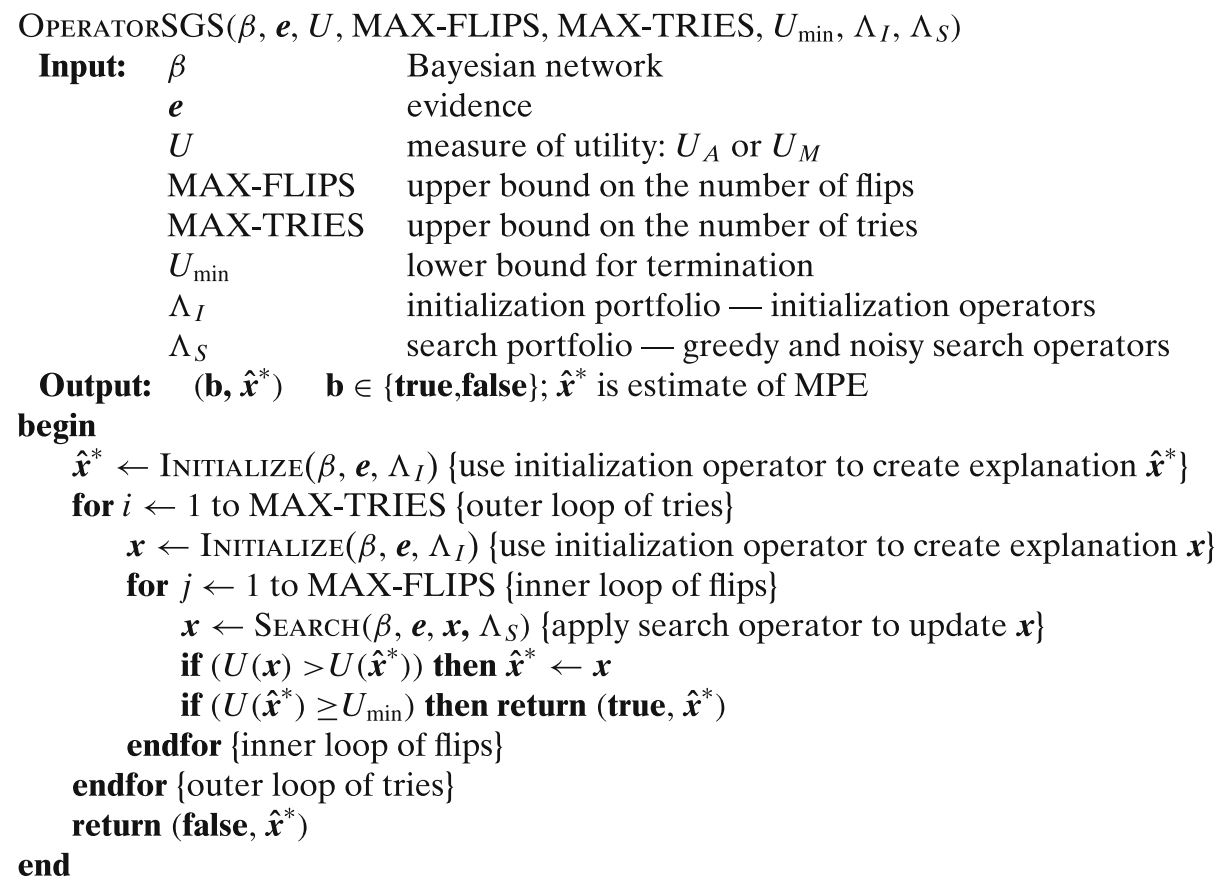

Fig. 5 The operator-based stochastic greedy search algorithm (OperatorSGS). OperatorSGS computes an MPE estimate $\hat{\boldsymbol{x}}^{*}$ given the input parameters, and operates in two main phases: an initialization phase and a local search phase. The initialization algorithm INITIALIZE applies initialization operators from $\Lambda_{I}$, while the search algorithm SEARCH applies search operators from $\Lambda_{S}$. OPERATORSGS terminates if an explanation $\hat{\boldsymbol{x}}^{*}$ of utility $U_{\min }$ or greater is found or if the computation exceeds MAX-TRIES tries 
SimpleSGS. We will return a more detailed discussion of OperatorSGS after formally introducing its initialization and search operators.

Definition 36 (Initialization operator, initialization operators) An initialization operator is a heuristic that computes, given a $\mathrm{BN} \beta$ and evidence $\boldsymbol{e}$, an explanation $\boldsymbol{x}$. A set of initialization operators is denoted $\Phi_{I}$.

The initialization operators that have so far been explored using OpERATORSGS are: uniform initialization (UN), forward simulation (FS), backward dynamic programming (BDP), and forward dynamic programming (FDP); we thus have $\Phi_{I}=$ $\{\mathrm{UN}, \mathrm{FS}, \mathrm{BDP}, \mathrm{FDP}\}[62]$. Other algorithms, for example the mini-bucket algorithm [41], can easily be incorporated as initialization operators in OPERATORSGS.

Definition 37 (Search operator, search operators) A (local) search operator is a heuristic that computes, for a BN $\beta$ with evidence $\boldsymbol{e}$ and an explanation $\boldsymbol{x}$, a new explanation $\boldsymbol{x}^{\prime}$. A one-flip (local) search operator is a search operator where $\boldsymbol{x}^{\prime}=$ $\boldsymbol{x}\left[X_{i} \leftarrow \bar{x}_{i}\right]$. A set of search operators is denoted $\Phi_{S}$.

Our search operators are closely related to flip selection strategies such as GSAT [78], WSAT-G [53], WSAT-B [53], WSAT [53, 77], Novelty [53], Novelty+, and DLM [75] in the SAT setting, and similar strategies in the BN setting [41, 48, $60,67,68]$. Traditionally, flip selection strategies are hard-coded using an if-thenelse statement containing a greedy part and a noisy part similar to what is done in SimpleSGS. In OperatorSGS, on the other hand, these two parts are typically represented by means of two distinct operators in a search portfolio. More importantly, OpERATORSGS is not restricted to two operators. We will return to the issue of flip selection strategies and search operators after introducing the following type of search operator.

Definition 38 (Compound operator) A compound operator is a two-tuple $(\Delta U(\boldsymbol{x}$, $\left.\left.x_{i, j}\right), C(\boldsymbol{A})\right)$, where $\Delta U\left(\boldsymbol{x}, x_{i, j}\right)$ is a gain function and $C(\boldsymbol{A})$ is a criterion of choice operating on a candidate array $\boldsymbol{A}$.

Example gain functions referred to in the above definition are additive gain $\Delta U_{A}\left(\boldsymbol{x}, x_{i, j}\right)$ and multiplicative gain $\Delta U_{M}\left(\boldsymbol{x}, x_{i, j}\right)$. Compound operators are used by CompoundSearch, see Fig. 6.

The operators $\{\mathrm{MG}, \mathrm{AG}, \mathrm{MN}, \mathrm{AN}\}$ are compound operators that we have used in OPERATORSGS so far; these operators are defined as follows.

Definition 39 (Compound operators MG, AG, MN, and AN) Using $C(\boldsymbol{A})=C_{G}(\boldsymbol{A})$ (the greedy criterion) we define these greedy search operators: ${ }^{6}$

$$
\begin{aligned}
\mathrm{MG} & =\left(\Delta U_{M}\left(\boldsymbol{x}, x_{i, j}\right), C_{G}(\boldsymbol{A})\right)\{\text { multiplicative gain with greedy criterion }\} \\
\mathrm{AG} & =\left(\Delta U_{A}\left(\boldsymbol{x}, x_{i, j}\right), C_{G}(\boldsymbol{A})\right)\{\text { additive gain with greedy criterion }\} .
\end{aligned}
$$

\footnotetext{
${ }^{6}$ In previous work [60] MG was called BM, AG was called GM, MN was called BS, AN was called GS.
} 


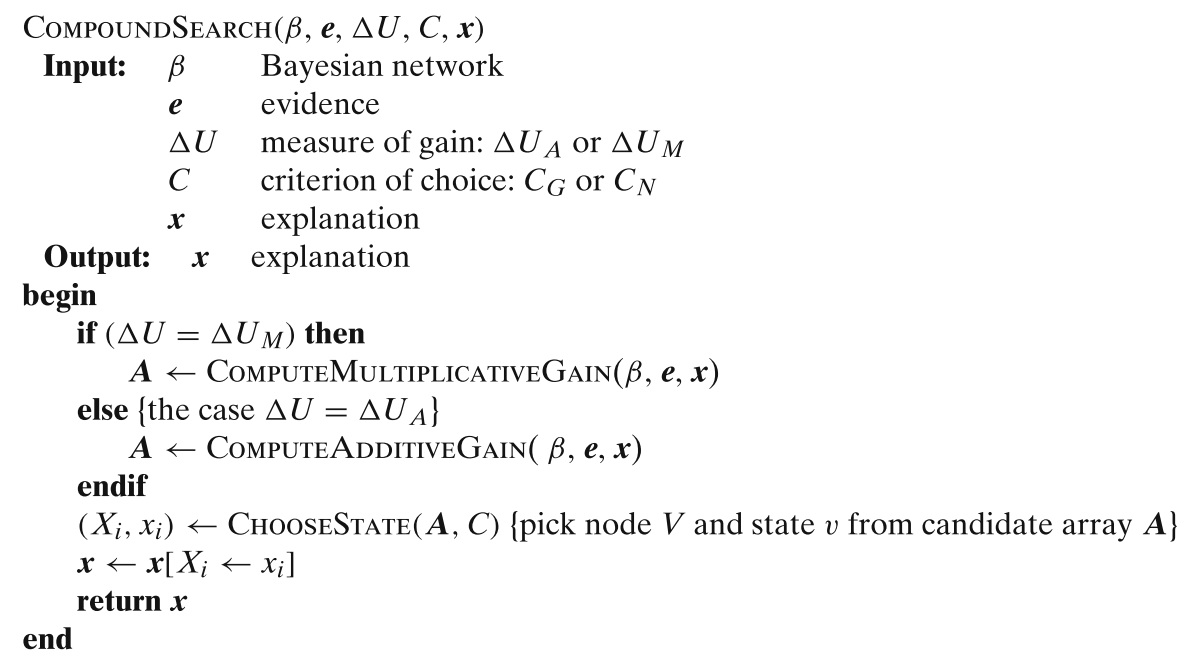

Fig. 6 An algorithm used by the SEARCH algorithm in OperatorSGS. It performs stochastic local search on the input explanation $\boldsymbol{x}$, using a compound operator defined by the input parameters $\Delta U$ and $C$. The result is an updated explanation $\boldsymbol{x}$, which is output

Using $C(\boldsymbol{A})=C_{N}(\boldsymbol{A})$ (the noisy criterion) we define these noisy search operators:

$$
\begin{aligned}
\mathrm{MN} & =\left(\Delta U_{M}\left(\boldsymbol{x}, x_{i, j}\right), C_{N}(\boldsymbol{A})\right)\{\text { multiplicative gain with noisy criterion }\} \\
\mathrm{AN} & =\left(\Delta U_{A}\left(\boldsymbol{x}, x_{i, j}\right), C_{N}(\boldsymbol{A})\right)\{\text { additive gain with noisy criterion }\} .
\end{aligned}
$$

The AG operator is formally justified by Theorems 27 and 32. Informally, there is the following relationship between maximizing GSAT gain and maximizing additive gain: Using GSAT gain, one flips the logic variable which gives the highest increase in the number of satisfied clauses [78]. Using the additive operator AG, we flip the $\mathrm{BN}$ node which gives the greatest additive increase in values of the affected CPTs.

In addition to the compound operators introduced in Definition 39, there are operators that are not formed by combining a gain function and a criterion of choice. For example, we have investigated the uniform noise (NU) and stochastic simulation (SS) operators. Uniform noise means that a non-evidence node, picked uniformly at random, is flipped with probability $p_{N}$. Stochastic simulation is Gibbs sampling in BNs, where nodes are sequentially sampled [71]. Altogether, this gives search operators $\Phi_{S}=\{\mathrm{NU}, \mathrm{SS}, \mathrm{MG}, \mathrm{AG}, \mathrm{MN}, \mathrm{AN}\}$.

An operator portfolio $\Lambda$, formally introduced in Definition 40 below, is generally speaking a set of operators along with an approach to operator selection. We focus here on selection according to a probability distribution defined by associating a probability with each operator as follows. ${ }^{7}$

\footnotetext{
${ }^{7} \mathrm{An}$ alternative to the stochastic portfolio is a round-robin portfolio or schedule. A round-robin portfolio is a sequence of $q$ operators $\Phi=\left(\phi_{0}, \ldots, \phi_{q-1}\right)$ such that the $j$-th time an operator is selected for execution from $\Phi$, operator $\phi_{i}$ where $i=j \bmod r$ is selected. We do not further investigate round-robin portfolios in this article.
} 
Definition 40 (Stochastic portfolio) Let $q \geq 1$ and let $\Phi=\left\{\phi_{1}, \ldots, \phi_{q}\right\}$ be a set of operators. A stochastic portfolio over $\Phi$ is a set of $q$ operator tuples $\Lambda=\left\{v_{1}, \ldots, v_{q}\right\}=$ $\left\{\left(\phi_{1}, p_{1}\right), \ldots,\left(\phi_{q}, p_{q}\right)\right\}$ where $0 \leq p_{i} \leq 1, \sum_{i=1}^{q} p_{i}=1$, and $\left(\phi_{i}, p_{i}\right)$ means that the $i$ th operator $\phi_{i}$, where $1 \leq i \leq q$, is selected (and then executed) with probability $p_{i}$ whenever some operator is selected from $\Lambda$.

Initialization algorithms have turned out to be important components in SGS as well as in other similar algorithms [41, 48, 62, 68]. In OperatorSGS, the input parameters therefore include a portfolio of initialization operators $\Lambda_{I}$, defined as follows.

Definition 41 (Initialization portfolio) Let $\Phi_{I}$ be a set of initialization operators. The OPERATORSGS initialization portfolio $\Lambda_{I}$ is a stochastic portfolio over $\Phi_{I}$ according to Definition 40.

The initialization algorithms construct explanations which make up starting points that the local search steps incrementally improve, leading to increasingly better MPE estimates. We have investigated the following initialization operators for stochastic generation of initial explanations: uniform initialization (UN), forward simulation (FS), forward dynamic programming (FDP), and backward dynamic programming (BDP) [62]. These stochastic initialization operators all have a time complexity of $O(n)$ in the number of nodes. A wide range of initialization heuristics can easily be incorporated as initialization operators in OPERATORSGS.

The portfolio $\Lambda_{S}$ for the local search heuristics is formally introduced as follows.

Definition 42 (Search portfolio) Let $\Phi_{S}$ be a set of search operators. The OpERATORSGS search portfolio $\Lambda_{S}$ is a stochastic portfolio over $\Phi_{S}$ according to Definition 40.

The input parameters of OperatorSGS, see Fig. 5, largely overlap those of SimpLESGS but include a portfolio of search operators $\Lambda_{S}$ and a portfolio of initialization operators $\Lambda_{I}$. OPERATORSGS chooses only among the operators included in the portfolios $\Lambda_{I}$ and $\Lambda_{S}$. More specifically, Initialize $\left(\beta, \boldsymbol{e}, \Lambda_{I}\right)$ returns a new explanation $\boldsymbol{x}$ for $\beta$, created using the initialization operators in the initialization portfolio $\Lambda_{I}$ (see Definition 41). SEARCH $\left(\beta, \boldsymbol{e}, \boldsymbol{x}, \Lambda_{S}\right.$ ) performs a search step on the explanation $\boldsymbol{x}$, using the measure of gain associated with the search operator chosen from the search portfolio $\Lambda_{S}$. A search operator is picked from the search portfolio $\Lambda_{S}$ as described in Definition 42. Compound operators are invoked by SEARCH using CompoundSearch, see Fig. 6.

This operator-based approach allows us to use a mixture of different operators, thus allowing us to emulate a wide range of SLS algorithms investigated previously. Here are a few examples from the literature.

Example 43 For computing SAT, Selman et al. investigated, in the mixed random walk strategy, greedy search (GSAT) combined with a random walk (RW) [77]: $\Lambda_{S}=$ $\left\{\left(\mathrm{GSAT}, p_{\mathrm{GSAT}}\right),\left(\mathrm{RW}, p_{\mathrm{RW}}\right)\right\}$. 
Table 1 Joint probability table decomposed according to the two orthogonal dimensions of (i) greedy versus noisy search and (ii) additive versus multiplicative gain computation. Taken together, these two dimensions define four operators or two-tuples of search operators (or heuristics) and probabilities as illustrated

\begin{tabular}{llc}
\hline & Greedy: $p_{G}=p_{\mathrm{AG}}+p_{\mathrm{MG}}$ & Noisy: $p_{N}=p_{\mathrm{AN}}+p_{\mathrm{MN}}$ \\
\hline Additive: & Additive $\&$ greedy: & Additive $\&$ noisy: \\
$p_{A}=p_{\mathrm{AG}}+p_{\mathrm{AN}}$ & (AG, $\left.p_{\mathrm{AG}}\right)$ & (AN, $\left.p_{\mathrm{AN}}\right)$ \\
Multiplicative: & Multiplicative $\&$ greedy: & Multiplicative \& noisy: \\
$p_{M}=p_{\mathrm{MG}}+p_{\mathrm{MN}}$ & $\left(\mathrm{MG}, p_{\mathrm{MG}}\right)$ & $\left(\mathrm{MN}, p_{\mathrm{MN}}\right)$ \\
\hline
\end{tabular}

Example 44 For computing MPE, Kask and Dechter investigated greedy search (GR) with stochastic simulation (SS) using mini-bucket (MB) initialization [41]: $\Lambda_{S}=\left\{\left(\mathrm{GR}, p_{\mathrm{GR}}\right),\left(\mathrm{SS}, p_{\mathrm{SS}}\right)\right\}$ and $\Lambda_{I}=\{(\mathrm{MB}, 1)\}$.

Example 45 For computing MAP, Park and Darwiche investigated (among a total of 11 SLS algorithms) hill-climbing (HILL) with random noise (NU), using MPE-based initialization (MPE) [68]: $\Lambda_{S}=\left\{\left(\right.\right.$ HiLL,$\left.\left.p_{\text {HILL }}\right),\left(\mathrm{NU}, p_{\mathrm{NU}}\right)\right\}$ and $\Lambda_{I}=\{(\mathrm{MPE}, 1)\}$.

For further examples, Schuurmans and Southey investigated eight flip selection strategies [75]; these could also be formulated as OpERATORSGS search portfolios. ${ }^{8}$

Turning to our additive approach, we now introduce a particular search portfolio which contains additive operators; see also Table 1.

Definition 46 Consider the compound operators from Definition 39 and suppose that $p_{\mathrm{AN}}+p_{\mathrm{AG}}+p_{\mathrm{MN}}+p_{\mathrm{MG}}=1$. We define $\Lambda_{S}\left(p_{\mathrm{AN}}, p_{\mathrm{AG}}, p_{\mathrm{MN}}\right)=\left\{\left(\mathrm{AN}, p_{\mathrm{AN}}\right)\right.$, $\left.\left(\mathrm{AG}, p_{\mathrm{AG}}\right),\left(\mathrm{MN}, p_{\mathrm{MN}}\right),\left(\mathrm{MG}, p_{\mathrm{MG}}\right)\right\}$, where $p_{\mathrm{MG}}=1-p_{\mathrm{AN}}-p_{\mathrm{AG}}-p_{\mathrm{MN}}$, as a search portfolio.

In Section 6.2 we will see that this search portfolio is very effective on application BNs. Also, the search portfolio in Definition 46 enables us to discuss an analogue between the probabilistic use of our additive measure and the use of noise in SLS. Varying the noise probability $p_{N}$, or the probability of applying a non-greedy heuristic, has been found to have a dramatic impact on SLS run time $[20,31,34,53,57,76,77]$. Utilizing our additive utility measure, we introduce the concept of additive probability $p_{A}$, or the probability of applying an additive heuristic, which is similar but orthogonal to noise probability $p_{N}$. In Definition 46 , only the operators AN and AG are additive, hence $p_{A}=p_{\mathrm{AN}}+p_{\mathrm{AG}}$. Varying the additive probability $p_{A}$ turns out to have a major impact on SLS computation time when searching for MPEs in partly deterministic BNs, as presented in Section 6.

\footnotetext{
${ }^{8}$ Schuurmans and Southey introduced a novel flip selection strategy called DLM; in addition they considered the existing strategies GSAT, HSAT, WSAT-G, WSAT-B, WSAT, Novelty, and Novelty+.
} 


\section{Analysis of Portfolios in Stochastic Local Search}

The goal of this section is to analyze SLS portfolio effects and the initialization and search portfolios of OPERATORSGS in particular. Given our observation that this operator-based approach can simulate many other SLS algorithms, including SIMPLESGS, the analysis is hopefully of broad interest and complements previous SLS analysis efforts $[32,57,62]$.

Section 5.1 discusses the incompleteness of SGS, and argues for the Las Vegas analysis and experimentation approach we have used in this article. We discuss our Markov chain-based analysis approach in Section 5.2, present results for the initialization portfolio in Section 5.3, and for the search portfolio in Section 5.4.

\subsection{Incompleteness, Termination, and Las Vegas Algorithms}

Stochastic local search algorithms are in general incomplete algorithms. Specifically, if there is no input parameter like SGS's $U_{\min }=\operatorname{Pr}\left(\boldsymbol{x}^{*}\right)$, one cannot say whether a current estimate $\hat{\boldsymbol{x}}^{*} \in \boldsymbol{X}^{*}$ or $\hat{\boldsymbol{x}}^{*} \notin \boldsymbol{X}^{*}$, and this raises the question of when to terminate search. Incomplete algorithms are appropriate when complete methods are impractical due to excessive demands on time, space, or both. For example, with limited space a complete algorithm like clique tree clustering might not even be able to compile a given $\mathrm{BN}[54,61,81]$. Alternatively, there might only be a fixed amount of time available that is too short for complete computation, and one might at the same time be willing to sometimes make a mistake. For example, there are situations where the cost of making an occasional mistake by finding $\hat{\boldsymbol{x}}^{*} \notin \boldsymbol{X}^{*}$, is small compared to the cost-in terms of space or time-needed to use a complete $\mathrm{BN}$ inference algorithm such as clique tree clustering.

Since SLS algorithms are randomized, their operation may be described using bivariate distributions with random variables for (i) the running time (in number of operations, say) and (ii) the current estimate of the utility of an optimal solution. Unfortunately, keeping track of both execution time and the quality of the MPE estimate complicates scientific analysis and experimentation. For simplicity, we generally take a Las Vegas approach [25] and use $U_{\min }=\operatorname{Pr}\left(\boldsymbol{x}^{*}\right)$, making SGS terminate only after an MPE is found. Clearly, in applications one will in general not know $\operatorname{Pr}\left(\boldsymbol{x}^{*}\right)$, and therefore cannot use $U_{\min }=\operatorname{Pr}\left(\boldsymbol{x}^{*}\right)$ as a termination criterion in SGS. Using $U_{\min }=\operatorname{Pr}\left(\boldsymbol{x}^{*}\right)$ might therefore by some readers be regarded as "cheating". However, in a scientific study such as the present we argue that using $U_{\min }=\operatorname{Pr}\left(\boldsymbol{x}^{*}\right)$ is entirely appropriate and indeed often to be preferred. First, it is the hardest test of SLS algorithms in terms of computing an explanation, since they are not allowed to terminate with $\hat{\boldsymbol{x}}^{*} \notin \boldsymbol{X}^{*}$. Second, since the SLS algorithm can terminate as soon as some $\hat{\boldsymbol{x}}^{*} \in \boldsymbol{X}^{*}$ is reached, one avoids additional complication induced by the termination criterion. In this manner, the effect of the initialization and search algorithms (our main interest here) is cleanly isolated from the effect of the termination criterion, which in applications might be quite involved.

The question of when to terminate SLS has, we believe, ultimately an applicationdependent answer. Certain applications, including real-time applications, have hard upper bounds on execution time and they might need to terminate MPE computation if that hard upper bound is exceeded. Other applications do not have hard real-time requirements and softer termination criteria would be appropriate. Giving specific 
recommendations beyond these general observations is unfortunately beyond the scope of this article.

\subsection{Markov Chains and Hitting Times}

We now introduce definitions of a few random variables that will be used to characterize SLS performance.

Definition 47 Let the flip length (number of SLS flips until termination) be a discrete random variable $F$. Let the run length (number of SLS operations, both initializations and flips, until termination) be a discrete random variable $R$.

There are in fact families of random variables involved here. These families are characterized by the $\eta$ input parameters of an SLS algorithm, a tuple $\left(\Theta_{1}=\theta_{1}, \ldots\right.$, $\Theta_{\eta}=\theta_{\eta}$ ). In OperatorSGS, $\eta=7$ and of particular interest are the initialization portfolio parameter $\Theta_{\eta-1}=\Theta_{6}=\Lambda_{I}$ as well as the search portfolio parameter $\Theta_{\eta}=$ $\Theta_{7}=\Lambda_{S}$. In SIMPLESGS we have $\eta=6$. Since $F$ depends on the parameter values $\theta=$ $\left(\theta_{1}, \ldots, \theta_{\eta}\right)$ used, we say $F(\boldsymbol{\theta})$ when we want to make this explicit. For example, if we consider MAX-FLIPS and vary $m$ in MAX-FLIPS $=m$ while keeping the remaining $\eta-1$ input parameters constant, we may write $F(m) ; R(m)$ is similar. In addition, we may condition on portfolio parameters $\Lambda_{I}$ and $\Lambda_{S}$ of OpERATORSGS; more about this below.

The search processes of SIMPLESGS and similar SLS algorithms can be analyzed using exact or approximate Markov chain models as follows (see also [32, 57]). Let us for simplicity assume $n$ binary non-evidence BN nodes such that an exact Markov chain model has $2^{n}$ states. This Markov chain is structured as a hypercube where each hypercube node $\boldsymbol{b}$ represents a bitstring. A state $\boldsymbol{b} \in\{0,1\}^{n}$ in such a Markov chain has $n$ neighbors, namely states that are one flip away.

Definition 48 (Neighborhood) Let $\boldsymbol{b}$ be a bitstring of length $n$. $\boldsymbol{b}$ 's neighbors $n(\boldsymbol{b})$, strict neighbors $n^{\prime}(\boldsymbol{b})$, and non-neighbors $\bar{n}(\boldsymbol{b})$ are defined as follows

$$
\begin{aligned}
n(\boldsymbol{b}) & =\left\{\boldsymbol{c} \in\{0,1\}^{n}\left|\sum_{i=1}^{n}\right| b_{i}-c_{i} \mid \leq 1\right\} \\
n^{\prime}(\boldsymbol{b}) & =n(\boldsymbol{b})-\{\boldsymbol{b}\} \\
\bar{n}(\boldsymbol{b}) & =\{0,1\}^{n}-n(\boldsymbol{b}) .
\end{aligned}
$$

We now introduce the notion of exact Markov chains over bitstrings; they will be useful when analyzing how SLS algorithms process problem instances.

Definition 49 (Exact Markov chain model) An exact Markov chain model $\mathcal{M}$ has states $\mathcal{S}=\left\{s_{0}, s_{1}, \ldots\right\}=\left\{\boldsymbol{b} \mid \boldsymbol{b} \in\{0,1\}^{n}\right\}$ and an initial probability vector $\mathcal{V}$ with $\operatorname{Pr}\left(A_{0}=s_{k}\right)=\pi_{k}$ for $1 \leq k \leq 2^{n}$. The transition probability matrix $\mathcal{P}$ is a stochastic matrix given by

$$
\begin{aligned}
& \operatorname{Pr}\left(A_{t+1}=\boldsymbol{c} \mid A_{t}=\boldsymbol{b}\right)=0 \text { if } \boldsymbol{c} \in \bar{n}(\boldsymbol{b}) \\
& \operatorname{Pr}\left(A_{t+1}=\boldsymbol{c} \mid A_{t}=\boldsymbol{b}\right) \geq 0 \text { if } \boldsymbol{c} \in n(\boldsymbol{b}) .
\end{aligned}
$$


For the uniform noise operator tuple (NU, $\left.p_{\mathrm{NU}}\right),(28)$ is clearly $\operatorname{Pr}\left(A_{t+1}=\boldsymbol{c} \mid A_{t}=\right.$ $\boldsymbol{b})=1 / n$ in the transition matrix $\mathcal{P}$ of an exact Markov chain model. Probabilities as introduced in Definitions 34 and 35 also define rows in transition matrixes $\mathcal{P}$.

To enable introduction of operators, we slightly extend the exact Markov chain notation in Definition 49 as follows. If there are $\xi$ different initialization operators, we have multiple initial probability vectors $\mathcal{V}_{i}$ with $\operatorname{Pr}\left(A_{i, 0}=s_{k}\right)$ for $1 \leq i \leq \xi$ and $1 \leq k \leq 2^{n}$. If there are $\chi$ search operators, we have multiple transition probability matrixes $\mathcal{P}_{j}$ for $1 \leq j \leq \chi$.

SimpleSGS and OperatorSGS can be analyzed using exact Markov chain models up to MAX-FLIPS flips, similar to the SimPLESLS algorithm [57]. In fact, using the exact Markov chain model, we can provide a sufficient condition for MPE computation using SIMPLESGS.

Theorem 50 Let MAX-TRIES $=\infty, M A X-F L I P S=\infty, p_{N}>0, U=U_{M}$, and $U_{\min }=\operatorname{Pr}\left(\boldsymbol{x}^{*}\right)$ in SimpleSGS. Then SimpleSGS is a Las Vegas algorithm and returns (true, $\left.\boldsymbol{x}^{*}\right)$ such that $\boldsymbol{x}^{*} \in \boldsymbol{X}^{*}$.

Proof Since MAX-TRIES $=\infty$, there are two if-then statements from which SIMPLESGS can return, and they both read "if $\left(U\left(\hat{\boldsymbol{x}}^{*}\right) \geqslant U_{\min }\right)$ then return (true, $\left.\hat{\boldsymbol{x}}^{*}\right)$." By substituting in assumptions, we obtain

$$
U\left(\hat{\boldsymbol{x}}^{*}\right)=U_{M}\left(\hat{\boldsymbol{x}}^{*}\right)=\operatorname{Pr}\left(\hat{\boldsymbol{x}}^{*}\right) \geqslant U_{\min }=\operatorname{Pr}\left(\boldsymbol{x}^{*}\right),
$$

which clearly only holds when $\operatorname{Pr}\left(\hat{\boldsymbol{x}}^{*}\right)=\operatorname{Pr}\left(\boldsymbol{x}^{*}\right)$. If SImPLESGS returns from its first if-then statement we are done. Suppose that SimpleSGS does not return from the first if-then statement. We argue that SimpleSGS will eventually return from the second if-then statement as follows. Consider the exact Markov chain model $\mathcal{M}=$ $(\mathcal{S}, \mathcal{V}, \mathcal{P})$ corresponding to the particular input parameters for SIMPLESGS. Pick any two distinct states $s_{i} \in \mathcal{S}$ and $s_{j} \in \mathcal{S}$. Since by assumption $p_{N}>0$, it is easy to see that $s_{i}$ and $s_{j}$ communicate. Thus, all states $\mathcal{S}$, including optimal states $\mathcal{O}$, are recurrent. Some optimal state $\boldsymbol{x}^{*} \in \mathcal{O}$, where $U_{\min }=\operatorname{Pr}\left(\boldsymbol{x}^{*}\right)$, will thus eventually be visited regardless of the initial state. When this happens, SimpleSGS will return (true, $\left.\boldsymbol{x}^{*}\right)$.

The proof of the following theorem, which provides a sufficient condition for MPE computation using OPERATORSGS, is similar to that of Theorem 50.

Theorem 51 Let $M A X-T R I E S=\infty, M A X-F L I P S=\infty,\left(\mathrm{NU}, p_{\mathrm{NU}}\right) \in \Lambda_{S}$ with $p_{\mathrm{NU}}>0, U=U_{M}$, and $U_{\min }=\operatorname{Pr}\left(\boldsymbol{x}^{*}\right)$ in OperatorSGS. Then OperatorSGS is $a$ Las Vegas algorithm and returns (true, $\left.\boldsymbol{x}^{*}\right)$ such that $\boldsymbol{x}^{*} \in \boldsymbol{X}^{*}$.

Due to the exponential growth of $\mathcal{V}$ and $\mathcal{P}$ as a function of $n$ in Definition 49, it is also of interest to formalize SLS behavior by means of approximate Markov chains. Let the current state of SGS search be $\boldsymbol{b}$, and consider how search brings us closer to or farther away from an MPE $\boldsymbol{b}^{*}$. In the following definition we assume for simplicity, but without loss of generality [57], that $\boldsymbol{b}^{*}=1 \ldots 1 ; u(\boldsymbol{b})$ is a function that counts the number of $1 \mathrm{~s}$, or in other words the correct states relative to $\boldsymbol{b}^{*}$, in $\boldsymbol{b}$. Clearly, when $u(\boldsymbol{b})=n$ we have $\boldsymbol{b}=\boldsymbol{b}^{*}$. 
Definition 52 (Positive and negative neighbors) Let the bitstring $\boldsymbol{b}$, of length $n$, represent the current state of search and let $\boldsymbol{b}^{*}=1 \ldots 1$ be the optimal bitstring. We then define positive neighbors $n^{+}(\boldsymbol{b})=\{\boldsymbol{c} \in n(\boldsymbol{b}) \mid u(\boldsymbol{c})>u(\boldsymbol{b})\}$ and negative neighbors $n^{-}(\boldsymbol{b})=\{\boldsymbol{c} \in n(\boldsymbol{b}) \mid u(\boldsymbol{c})<u(\boldsymbol{b})\}$.

In an approximate Markov chain model, denoted a basic random walk in Fig. 7, we introduce states that correspond to all counts of the number of correct bits $u(\boldsymbol{b})$. Clearly, $0 \leq u(\boldsymbol{b}) \leq n$. With the exception of the two boundary states 0 and $n$, each state $i$ has two neighbors $i-1$ and $i+1$. Therefore we have a Markov chain model with $n+1$ states in which each state has two neighbors; a so-called random walk.

Can Markov chains such as random walks be applied also in the portfolio setting, and specifically to analyze OpERATORSGS? As we will see in the following, the answer to this question is "yes"; we first illustrate our analysis by means of an example.

Example 53 Suppose that we have a $\mathrm{BN} \beta$ with $n=5$ binary nodes. For OperATORSGS, let $\Lambda_{I}=\left\{\left(\phi_{1}, p_{1}\right),\left(\phi_{2}, p_{2}\right),\left(\phi_{3}, p_{3}\right)\right\}$ and $\Lambda_{S}=\left\{\left(\omega_{1}, q_{1}\right),\left(\omega_{2}, q_{2}\right)\right\}$. Figure 7 shows an augmented Markov chain model $\mathcal{M}$ (see also Definition 54) for OPERATORSGS processing $\beta$, given these portfolios $\Lambda_{I}$ and $\Lambda_{S}$.

In addition to states representing the underlying search space, corresponding to the states in a basic random walk model, our novel augmented Markov chain model includes states that represent the search and initialization operators-see Fig. 7. The advantage of this model is that it allows us to analyze search and initialization operators within the same framework as the underlying search space.

Basic Random Walk:

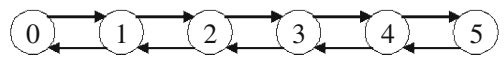

Augmented Random Walk:

States for Search Operator 1

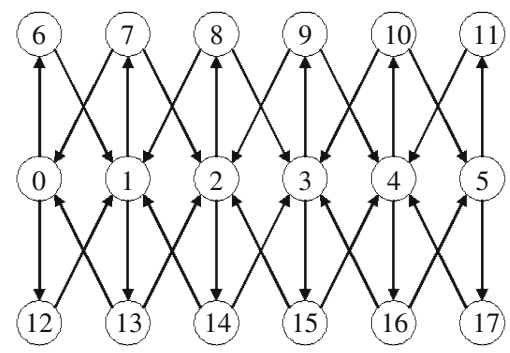

States for Search Operator 2

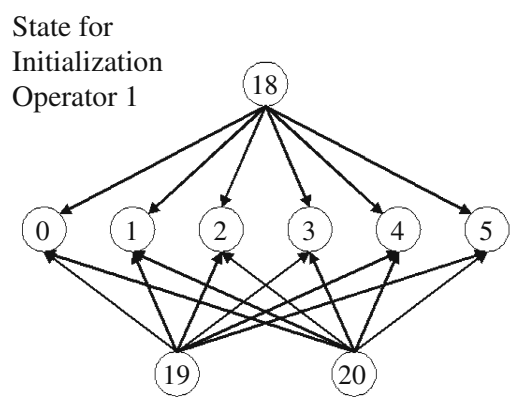

State for Initialization State for Initialization Operator 2 Operator 3

Fig. 7 An example of a basic random walk (top) and a corresponding augmented random walk model (bottom) for OPERATORSGS and other stochastic local search algorithms that use initialization and search portfolios. To reduce clutter, the states for the underlying search space are shown twice, namely in the context of the search portfolio with $\chi=2$ operators (bottom left) and in the context of the initialization portfolio with $\xi=3$ operators (bottom right). See also Example 53 
The details for Example 53 are as follows. We first consider states $\mathcal{S}$. The number of states in $\mathcal{S}$ is $k=(n+1)(\chi+1)+\xi=6 \times 3+3=21$. Among these, states $\{0, \ldots$, $5\}$ represent OPERATORSGS search right before search operator selection. States $\{6$, $\ldots, 11\}$ represent search right after selection of search operator $\omega_{1}$; states $\{12, \ldots$, 17\} represent search right after selection of search operator $\omega_{2}$. States 18,19 , and 20 represent selection of initialization operators $\phi_{1}, \phi_{2}$, and $\phi_{3}$ respectively. In $\mathcal{V}$, $\operatorname{Pr}\left(B_{0}=i\right)=0$ for $0 \leq i \leq(n+1)(\chi+1)-1=17$ and $\operatorname{Pr}\left(B_{0}=17+j\right)=p_{j}$ for $1 \leq$ $j \leq 3$, where $p_{j}$ are the initialization operator selection probabilities in Example 53 . In $\mathcal{P}$, we have $\operatorname{Pr}\left(B_{t+1}=i \mid B_{t}=j\right) \geq 0$ as indicated by edges in Fig. 7. For all other entries in $\mathcal{P}, \operatorname{Pr}\left(B_{t+1}=i \mid B_{t}=j\right)=0$.

We now formally introduce our novel Markov chain construction that clearly reflects the initialization and search portfolios of OPERATORSGS. This construction is illustrated in Example 53. We will in Theorem 55 formally show that a Markov chain is created.

Definition 54 (Augmented random walk) Consider a $\mathrm{BN}$ with $n$ binary nodes. Suppose that we have an initialization portfolio $\Lambda_{I}=\left\{\left(\phi_{i}, p_{i}\right) \mid 1 \leq i \leq \xi\right\}$ with initial probability vectors $\mathcal{V}_{i}$ for $1 \leq i \leq \xi$ and a search portfolio $\Lambda_{S}=\left\{\left(\omega_{j}, q_{j}\right) \mid 1 \leq j \leq \chi\right.$ \} with transition probability matrixes $\mathcal{P}_{j}$ for $1 \leq j \leq \chi$. The augmented random walk model $(\mathcal{S}, \mathcal{V}, \mathcal{P})$ is defined as follows. Put $m=(n+1)$ and $\ell=m(\chi+1)$. $\mathcal{M}$ has $k=\ell+\xi=|\mathcal{S}|$ discrete states named $\{0, \ldots, k-1\}$. The $k$-dimensional vector $\mathcal{V}$ of initial probabilities is defined as $\operatorname{Pr}\left(B_{0}=i\right)=0$ for $0 \leq i \leq \ell-1$ and $\operatorname{Pr}\left(B_{0}=i\right)=$ $p_{i-\ell+1} \geq 0$ for $\ell \leq i \leq k-1$. The potentially non-zero entries in the $k \times k$ matrix $\mathcal{P}$ of conditional probabilities are defined as:

$$
\begin{aligned}
\operatorname{Pr}\left(B_{t+1}=i \mid B_{t}=j+\ell-1\right) & =\sum_{\{\boldsymbol{b} \mid u(\boldsymbol{b})=i\}} \operatorname{Pr}\left(A_{j, 0}=\boldsymbol{b}\right), 0 \leq i \leq n \text { and } 1 \leq j \leq \xi \\
\operatorname{Pr}\left(B_{t+1}=j m+i \mid B_{t}=i\right) & =q_{j}, 0 \leq i \leq n \text { and } 1 \leq j \leq \chi \\
\operatorname{Pr}\left(B_{t+1}=i+1 \mid B_{t}=j m+i\right) & =\sum_{\left\{\boldsymbol{b}^{+} \mid \boldsymbol{b}^{+} \in n^{+}(\boldsymbol{b})\right\}} \operatorname{Pr}\left(A_{j, t+1}=\boldsymbol{b}^{+} \mid A_{j, t}=\boldsymbol{b}\right) \\
\operatorname{Pr}\left(B_{t+1}=i-1 \mid B_{t}=j m+i\right) & =\sum_{\left\{\boldsymbol{b}^{-} \mid \boldsymbol{b}^{-} \in n^{-}(\boldsymbol{b})\right\}} \operatorname{Pr}\left(A_{j, t+1}=\boldsymbol{b}^{-} \mid A_{j, t}=\boldsymbol{b}\right),
\end{aligned}
$$

where in (31) and (32) we have $0<i \leq n$ and $1 \leq j \leq \chi$. The remaining entries in $\mathcal{P}$ are zero.

We now discuss Definition 54 in more detail; see also Fig. 8. There is a oneto-one mapping between the $\xi$ last states in the augmented random walk model and the tuples in the initialization portfolio $\Lambda_{I}=\left\{\left(\phi_{1}, p_{1}\right), \ldots,\left(\phi_{\xi}, p_{\xi}\right)\right\}$. These $\xi$ initialization (operator) states are shown at the bottom in Fig. 8; the probabilities of the edges from these states are given by (29). There is also a relationship between states $0 \leq i \leq(n+1)(\chi+1)$ and the tuples in the search portfolio $\Lambda_{S}=\left\{\left(\omega_{1}, q_{1}\right), \ldots\right.$, $\left.\left(\omega_{\chi}, q_{\chi}\right)\right\}$ : States $0 \leq i \leq n$, which we will call (search operator) selection states, correspond to the moment, in OPERATORSGS, right before search operator selection from $\Lambda_{S}$ according to (30). States $(n+1) \leq i \leq(n+1)(\chi+1)$ are called (search operator) application states. Each of these latter states correspond to a time when a search operator has been picked from $\Lambda_{S}$, and is about to be applied by OpERATORSGS. Once 


\section{Basic Random Walk:}

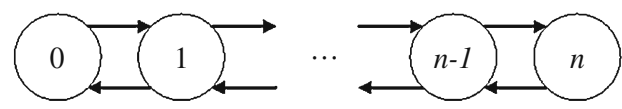

Augmented Random Walk:

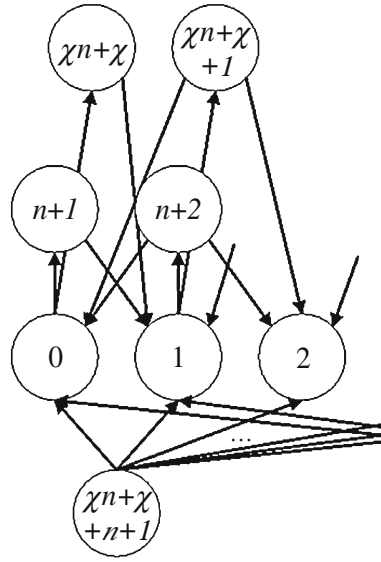

State for Initialization Operator 1

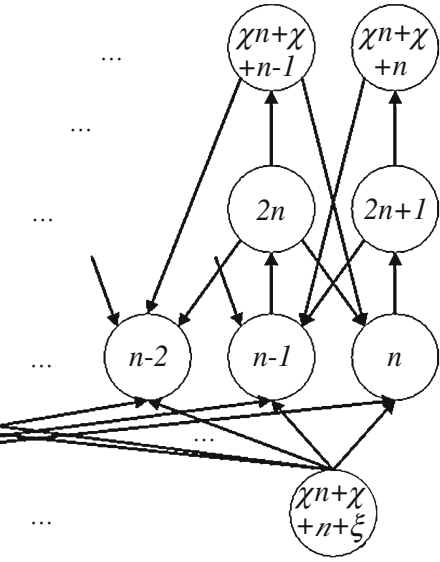

State for Initialization Operator $\xi$
Search Operator Application States for Search Operator $\chi$

Search Operator Application States for Search Operator 1

Random Walk States or Search Operator Selection States

Initialization Operator States

Fig. 8 The general constructions for a basic random walk model (top) and a corresponding augmented random walk model (bottom) for stochastic local search algorithms that use initialization and search portfolios

an operator has been selected and applied using (31) and (32), the Markov chain is again in a selection state $0 \leq i \leq n$, and the selection-application cycle continues until restart or termination.

There are two options for Markov chain analysis of our portfolio approach. First, we can perform analysis directly on the augmented random walk. Second, we can use the augmented random walk merely as an aid in constructing the basic random walk, and perform analysis on the basic random walk. In either case, since we have a Markov chain, Markov chain analysis techniques can be applied. In the rest of this work our main emphasis is on analysis directly on the augmented random walk, as reflected in the following result.

Theorem 55 An augmented random walk model $(\mathcal{S}, \mathcal{V}, \mathcal{P})$ induces a Markov chain $\mathcal{M}=(\mathcal{S}, \mathcal{V}, \mathcal{P})$ over the random variables $\left(B_{t}, t \geq 0\right)$.

Proof We consider $\mathcal{S}, \mathcal{V}$, and $\mathcal{P}$ in turn. $\mathcal{S}$ has $k=(n+1)(\chi+1)+\xi=\ell+\xi$ states and obviously defines a finite state space. $\mathcal{V}$ is given by $\left(\pi_{0}, \ldots, \pi_{\ell+\xi-1}\right)$, where

$$
\sum_{i=0}^{\ell+\xi-1} \pi_{i}=\sum_{i=0}^{\ell-1} \pi_{i}+\sum_{i=\ell}^{\ell+\xi-1} \pi_{i}=\sum_{j=1}^{\xi} p_{i}=1
$$


by the definition of $\Lambda_{I}=\left\{\left(\phi_{1}, p_{1}\right), \ldots,\left(\phi_{\xi}, p_{\xi}\right)\right\}$. For $\mathcal{P}$ 's $\xi$ initialization states we have for any $1 \leq j \leq \xi$ :

$$
\begin{aligned}
\sum_{i=0}^{\ell+\xi-1} \operatorname{Pr}\left(B_{t+1}=i \mid B_{t}=j+\ell-1\right) & =\sum_{i=0}^{n} \operatorname{Pr}\left(B_{t+1}=i \mid B_{t}=j+\ell-1\right) \\
& =\sum_{\{\boldsymbol{b} \mid u(\boldsymbol{b})=i\}} \operatorname{Pr}\left(A_{j, 0}=\boldsymbol{b}\right)=1
\end{aligned}
$$

which follows from (29) and the fact that $A_{j, 0}$ is an initial probability vector in an exact Markov chain model. For $\mathcal{P}$ 's $n+1$ (search operator) selection states we have for any $0 \leq i \leq n$ :

$$
\sum_{j=0}^{\ell+\xi-1} \operatorname{Pr}\left(B_{t+1}=j \mid B_{t}=i\right)=\sum_{j=1}^{\chi} \operatorname{Pr}\left(B_{t+1}=j m+i \mid B_{t}=i\right)=\sum_{j=1}^{\chi} q_{j}=1,
$$

which follows from (30) and the definition $\Lambda_{S}=\left\{\left(\omega_{1}, q_{1}\right), \ldots,\left(\omega_{\chi}, q_{\chi}\right)\right\}$. Finally, for $\mathcal{P}$ 's $\chi(n+1)$ (search operator) application states we have for $1 \leq j \leq \chi$ and $0 \leq i \leq n$ :

$$
\begin{aligned}
\sum_{k=0}^{\ell+\xi-1} \operatorname{Pr}\left(B_{t+1}=k \mid B_{t}=j m+i\right)= & \sum_{k=0}^{n} \operatorname{Pr}\left(B_{t+1}=k \mid B_{t}=j m+i\right) \\
= & \operatorname{Pr}\left(B_{t+1}=i+1 \mid B_{t}=j m+i\right) \\
& +\operatorname{Pr}\left(B_{t+1}=i-1 \mid B_{t}=j m+i\right),
\end{aligned}
$$

and by substituting (31) and (32) into the above we obtain:

$$
\begin{aligned}
\sum_{k=0}^{\ell+\xi-1} \operatorname{Pr}\left(B_{t+1}=k \mid B_{t}=j m+i\right)= & \sum_{\left\{\boldsymbol{b}^{+} \mid \boldsymbol{b}^{+} \in n^{+}(\boldsymbol{b})\right\}} \operatorname{Pr}\left(A_{j, t+1}=\boldsymbol{b}^{+} \mid A_{j, t}=\boldsymbol{b}\right) \\
& +\sum_{\left\{\boldsymbol{b}^{-} \mid \boldsymbol{b}^{-} \in n^{-}(\boldsymbol{b})\right\}} \operatorname{Pr}\left(A_{j, t+1}=\boldsymbol{b}^{-} \mid A_{j, t}=\boldsymbol{b}\right)=1,
\end{aligned}
$$

where the last equality follows because $\sum_{\boldsymbol{c} \in n^{\prime}(\boldsymbol{b})} \operatorname{Pr}\left(A_{j, t+1}=\boldsymbol{c} \mid A_{j, t}=\boldsymbol{b}\right)=1$ in an exact Markov chain model and $n^{\prime}(\boldsymbol{b})$ can be partitioned into $n^{+}(\boldsymbol{b})$ and $n^{-}(\boldsymbol{b})$. Since we have now shown that (i) $\mathcal{V}$ is a valid initial probability vector of size $k=|\mathcal{S}|$ and (ii) all of $\mathcal{P}$ 's $\xi+n+1+\chi(n+1)=k$ rows sum to one and hence $\mathcal{P}$ is a valid $k \times k$ conditional probability matrix, the desired result follows.

For SLS algorithms, one is typically interested in expected run time and its minimization. In this article, we study the expected hitting time $h(\boldsymbol{\theta})$. For our augmented random walk, the concept of expected hitting time is well-defined since the walk is a Markov chain. Given our results on additive utility and gain in Section 3 we are in particular interested in $h\left(p_{A}\right)$, were $p_{A}$ is the probability of applying an additive operator in the search portfolio. We now turn to hitting time minimization and define $\boldsymbol{\theta}^{*}=\arg \min h(\boldsymbol{\theta})$ and in particular $p_{A}^{*}=\arg \min h\left(p_{A}\right)$. The general problems of efficiently computing $\boldsymbol{\theta}^{*}$ and $p_{A}^{*}$ are topics for ongoing research. In the rest of this 
section we provide a few analytical results; in addition there are substantial empirical results as reflected in the experiments of this article.

\subsection{Analysis of Initialization}

We now turn to an analysis of the OPERATORSGS initialization portfolio. We consider different types of initialization portfolios, and introduce the following terminology.

Definition 56 Let $\Lambda$ be a stochastic portfolio. If $|\Lambda|=1$ then we call $\Lambda$ a homogeneous portfolio. If $|\Lambda| \geq 2$ then we call $\Lambda$ a heterogeneous portfolio.

Instead of varying the size of a portfolio, as implied by Definition 56, one can set selection probabilities to zero. For example, a portfolio with exactly one operator is obviously equivalent to a portfolio in which exactly one operator has selection probability one while all other operators have selection probability zero. For simplicity we exclude portfolios in which operators have zero probability of selection in our analysis in this section.

We introduce a random variable $\Phi$ to represent OPERATORSGS's random selection of an initialization operator from $\Lambda_{I}$. Suppose there are $\xi$ operators in the initialization portfolio. Given $\Phi$ and the random variable $F$ representing the number of flips, we introduce conditional probabilities $\operatorname{Pr}\left(F \mid \Phi=\phi_{i}\right)$ and conditional expectations $E\left(F \mid \Phi=\phi_{i}\right)$ for $0 \leq i \leq \xi$. For the special case of MAX-FLIPS $=\infty$, we have

$$
E\left(F \mid \Phi=\phi_{i}\right)=E\left(F \mid B_{0}=j\right)=E\left(T \mid B_{0}=j\right),
$$

where $j=i+(n+1)(\chi+1)-1$.

We may consider different initialization portfolios of the form $\Lambda_{I}=\left\{\left(\phi_{1}, p_{1}\right)\right.$, $\left.\left(\phi_{2}, p_{2}\right), \ldots,\left(\phi_{\xi}, p_{i}\right)\right\}$. Of particular interest is the optimal initialization portfolio $\Lambda_{I}^{*}$, formally defined as follows.

Definition 57 (Optimal initialization) The optimal initialization portfolio $\Lambda_{I}^{*}=$ $\left\{\left(\phi_{1}, p_{1}^{*}\right),\left(\phi_{2}, p_{2}^{*}\right), \ldots,\left(\phi_{\xi}, p_{\xi}^{*}\right)\right\}$ is defined by its optimal probabilities

$$
\boldsymbol{p}^{*}=\left(p_{1}^{*}, \ldots, p_{\xi}^{*}\right)=\arg \min _{p_{1}, \ldots, p_{\xi}}\left(\sum_{i=1}^{\xi} E\left(F \mid \Phi=\phi_{i}\right) p_{i}\right) .
$$

Clearly, the challenge here is to find $\boldsymbol{p}^{*}$. It turns out that homogeneous initialization portfolios, defined as $\Lambda_{I, 1}=\left\{\left(\phi_{1}, 1\right)\right\}, \Lambda_{I, 2}=\left\{\left(\phi_{2}, 1\right)\right\}, \ldots, \Lambda_{I, \xi}=\left\{\left(\phi_{\xi}, 1\right)\right\}$, play a central role as reflected in the following result.

Theorem 58 Let $\xi$ be the number of operators in the initialization portfolio. Consider, for $1 \leq i \leq \xi, E\left(F \mid \Phi=\phi_{i}\right) .{ }^{9}$ Suppose that $E\left(F \mid \Phi=\phi_{i}\right) \neq E\left(F \mid \Phi=\phi_{j}\right)$ for all $1 \leq i, j \leq \xi$ where $i \neq j$. Then there is a unique optimal initialization portfolio $\Lambda_{I}^{*}$, namely some homogeneous portfolio $\Lambda_{I, i}$ for $1 \leq i \leq \xi$.

\footnotetext{
${ }^{9}$ Note that $E\left(T \mid \Phi=\phi_{i}\right)$ is a number while $E(T \mid \Phi)$ is a random variable. In the former case the initialization portfolio $\Phi$ is given as a specific portfolio $\phi_{i}$, while in the latter case $\Phi$ is not specified.
} 
Proof Since $E\left(F \mid \Phi=\phi_{i}\right) \neq E\left(F \mid \Phi=\phi_{j}\right)$ for $1 \leq i, j \leq \xi$ where $i \neq j$, we assume without loss of generality the strict ordering of homogenous portfolios $E(F \mid \Phi=$ $\left.\phi_{1}\right)<E\left(F \mid \Phi=\phi_{2}\right)<\cdots<E\left(F \mid \Phi=\phi_{\xi}\right)$. Consider now the expectation $E(F)$ for an arbitrary heterogeneous initialization portfolio $\left\{\left(\phi_{1}, p_{1}\right),\left(\phi_{2}, p_{2}\right), \ldots,\left(\phi_{\xi}, p_{\xi}\right)\right\}$. Using the law of conditional expectation gives

$$
E(F)=\sum_{i=1}^{\xi} E\left(F \mid \Phi=\phi_{i}\right) \operatorname{Pr}\left(\Phi=\phi_{i}\right)=\sum_{i=1}^{\xi} E\left(F \mid \Phi=\phi_{i}\right) p_{i} .
$$

By our strict ordering assumption of the homogenous portfolios, we can for $2 \leq$ $i \leq \xi$ write $E\left(F \mid \Phi=\phi_{i}\right)=E\left(F \mid \Phi=\phi_{1}\right)+c_{i}$ for increasing constants $c_{i}>0$. For convenience, we define $c_{1}=0$. Now, (33) can be written as

$$
\begin{aligned}
E(F) & =\sum_{i=1}^{\xi}\left(E\left(F \mid \Phi=\phi_{1}\right)+c_{i}\right) p_{i} \\
& =\sum_{i=1}^{\xi} E\left(F \mid \Phi=\phi_{1}\right) p_{i}+\sum_{i=1}^{\xi} c_{i} p_{i} \\
& =E\left(F \mid \Phi=\phi_{1}\right)+\sum_{i=1}^{\xi} c_{i} p_{i} .
\end{aligned}
$$

There are now two cases to consider. Case (i): $p_{1}=1$. From (34) it is easy to see that $E(F)=E\left(F \mid \Phi=\phi_{1}\right)$ since $p_{i}=0$ for $i \neq 1$. Case (ii): $p_{1}<1$. From (34) it follows that $E(F)>E\left(F \mid \Phi=\phi_{1}\right)$, since clearly $\sum_{i=1}^{\xi} c_{i} p_{i}>0$. Consequently, $E\left(F \mid \Phi=\phi_{1}\right)$ is the minimal expectation, and the homogeneous initialization portfolio containing exactly one operator is optimal, and specifically $\Lambda_{I}^{*}=\Lambda_{I, 1}=\left\{\left(\phi_{1}, 1\right)\right\}$.

The implication of Theorem 58 is that once parameters $\boldsymbol{\theta}$ have been fixed, then best performance is ensured by using a homogeneous initialization portfolio. Some readers might regard Theorem 58 as a negative result for initialization portfolios and might ask why we consider them in the first place. Here is why. First, Theorem 58 could not have been derived unless an initialization portfolio was assumed. Second, one is typically interested in performing inference over distributions of BNs, not one particular BN. And for different BNs, different initialization algorithms often turn out to be optimal, and the use of an initialization portfolio supports the desired flexibility in the use of initialization algorithms. Third, even though one initialization algorithm among many is optimal, it is often not a priori obvious which one it is. So there is a pre-processing phase, during which different initialization operators are considered, and the existence of an initialization portfolio enables this preprocessing phase.

\subsection{Analysis of Search}

We now turn our attention to SLS operations and assume that MAX-TRIES $=\infty$ and that the initialization portfolio has been fixed. In OpERATORSGS an operation is executed when an operator is picked from either $\Lambda_{S}$ or $\Lambda_{I}$ and then run. In SimpleSGS an operation is executed when InITIALIZE is called in the loop of tries and when SEARCH is called in the loop of flips. 
The expected number of operations executed, including both initializations and local search steps, is characterized by the following result (see [62] for the derivation).

Theorem 59 (Expected number of operations) Suppose that $\operatorname{Pr}(R(m) \leq m)>0$ and let $M A X-F L I P S=m$. The expected number of $S L S$ operations executed, $E(R(m))$, is given by

$$
E(R(m))=\frac{m(1-\operatorname{Pr}(F(\infty) \leq m))+\sum_{i=0}^{m} i \operatorname{Pr}(F(\infty)=i)+1}{\operatorname{Pr}(F(\infty) \leq m)}
$$

Theorem 59 is a generalization of previous results $[69,75]$ in that it accounts for all operators including initialization operators in $\Lambda_{S}$.

Our analysis in the rest of this section assumes an SLS model $(\mathcal{M}, \mathcal{O})$, where $\mathcal{M}=$ $(\mathcal{S}, \mathcal{V}, \mathcal{P})$ is a Markov chain. Further, $T$ is (as before) a random variable representing $\mathcal{M}$ 's hitting time. The benefit of a Markov chain analysis is illustrated as follows. Suppose that we vary only the $\Lambda_{S}$ part in $\theta$, and further suppose that $\Lambda_{S}$ contains only two operators, namely a noisy operator (applied with probability $p_{N}$ ) and greedy operator (applied with probability $p_{G}=1-p_{N}$ ). In related work we have shown that the expected hitting time $h$ as a function of $p_{N}, h\left(p_{N}\right)$, are rational functions, or in other words ratios of polynomials $P\left(p_{N}\right)$ and $Q\left(p_{N}\right): h\left(p_{N}\right)=P\left(p_{N}\right) / Q\left(p_{N}\right)$ [57]. The curves for $h\left(p_{N}\right)$ are analytical counterparts to and explain theoretically the so-called noise response curves that have been extensively studied empirically [57]. The impact of noise varies with $\mathrm{BN}$ hardness, with optimal noise level increasing with increasing problem hardness.

Analogously, we consider here hitting time as a function of additivity $p_{A}$, with corresponding (empirical) additive response curves investigated in Section 6. Consider an SLS model $(\mathcal{M}, \mathcal{O})$, where the Markov chain $\mathcal{M}=(\mathcal{S}, \mathcal{V}, \mathcal{P})$ is defined over a bitstring of length $n$ and with parameter $p_{A}$. Let $\kappa=n+1$, assume optimum states $\left\{s_{\lambda}, \ldots, s_{\kappa}\right\}=\mathcal{O}$ where $\lambda \leq \kappa$, and form a system of equations for $\mathcal{M}$ 's expected first passage times $m_{i}$ for $1 \leq i \leq \kappa$. There exists an equivalent upper triangular system $\boldsymbol{U} \boldsymbol{m}=\boldsymbol{b}$, where $\boldsymbol{m}=\left(m_{1}, \ldots, m_{\lambda-1}\right)^{T}$, in which all coefficients in $\boldsymbol{U}$ and $\boldsymbol{b}$ are rational functions of $p_{A}$. Performing back substitution on $\boldsymbol{U} \boldsymbol{m}=\boldsymbol{b}$, we obtain the following expected hitting time result.

Theorem 60 (Rationality of hitting time) Consider an $S L S$ model $(\mathcal{M}, \mathcal{O})$, where $\mathcal{M}=(\mathcal{S}, \mathcal{V}, \mathcal{P})$ is an augmented Markov chain defined over a bitstring of length $n$ and with parameter $p_{A}$. The expected hitting time for $\mathcal{M}$ is a rational function of $p_{A}$, $h\left(p_{A}\right)=P\left(p_{A}\right) / Q\left(p_{A}\right)$, where $P\left(p_{A}\right)$ and $Q\left(p_{A}\right)$ are polynomials.

The proof of Theorem 60 is similar to that of a previous result [57], except that (i) it concerns $p_{A}$ rather than $p_{N}$, and (ii) is based on our approximate (see Definition 54) rather than an exact Markov (see Definition 49) chain model. In the proof of the theorem, the key idea is to perform Gaussian elimination symbolically, such that the parameter $p_{A}$ is preserved throughout the derivation.

Theorem 60, which is concerned with a single $\mathrm{BN}$, can be extended in a natural way to multiple BNs by means of finite mixture distributions. Again, we consider additive probability to be the independent parameter. Because of closure properties [57], it follows from Theorem 60 that the SLS hitting time for a mixture of BNs is also a rational function $h\left(p_{A}\right)=P\left(p_{A}\right) / Q\left(p_{A}\right)$. To approximate the rational functions 
induced by single BNs as well as multiple BNs we have used low-order polynomials [57]; this approach is motivated by Weierstrass' theorem and supported by empirical results.

\section{Experiments}

We now turn to experiments performed with the SGS system, which implements the SimpleSGS and OperatorSGS algorithms [54, 57, 60, 62]. In these experiments, we attempt to carefully balance scientific and competitive experimentation [30]. In other words, we aim to complement our analytical results, and in particular improve the understanding of our portfolio approach as well as that of additive utility and gain (scientific experimentation [30]). For two reasons, we study SGS in depth rather than investigate a large number of SLS systems at a more superficial level. First, apart from SGS we know of no SLS system for MPE computation that implements the additive approach. Second, there is clear evidence that a problem instance that is hard for one SLS algorithms is also hard for other SLS algorithms [34].

On the competitive experimentation side, we investigate the question of how our SLS algorithms, as implemented by SGS, compare to exact algorithms, in particular clique tree clustering as implemented in the state-of-the-art tree clustering system Hugin $[14,45]$. Hugin uses two phases to compute an MPE, a compilation phase and an execution phase. Hugin was chosen since (i) we wanted to employ an exact algorithms to compute MPEs (as opposed to approximations) and (ii) HuGIN uses one of the best exact methods, the tree clustering algorithm. In our experiments, we used a Las Vegas methodology, motivated in Section 5.1, which has also been used extensively in experimental research on satisfiability [64]: HugIN was always used before SGS, in order to find an MPE $\boldsymbol{x}^{*}$ if one existed. The reason for focusing on instances which HugIn could process is as follows. For these cases we know for sure that some non-zero MPE $\boldsymbol{x}^{*}$ exists and can therefore input $U_{\min }=\operatorname{Pr}\left(\boldsymbol{x}^{*}\right)$ to SGS to force convergence to an MPE. In addition, and following what is typically done in experiments, we assume that HuGIN's compilation time as well as SGS's parameter optimization time can be amortized over a large number of MPE queries to a BN.

In the rest of this section we provide experimental results for SGS and compare SGS to Hugin. We use synthetic BNs in Section 6.1 and BNs from applications in Section 6.2. For the experiments discussed here, a Dell Dimension $4500 \mathrm{CPU}, 2 \mathrm{GHz}$ Intel Pentium IV using $1 \mathrm{~GB}$ of RAM and up to $2 \mathrm{~GB}$ of swap space has been used. The computer was running Windows XP.

\subsection{Experiments with Synthetic Bayesian Networks}

We now compare SGS to HugIN, using synthetically generated BNs. The experiments reported in this section focused on varying the $C / V$-ratio in BNs, using synthetic networks where the hardness of MPE computation can be controlled. These results provide insights into the general patterns of performance of a stochastic local search algorithm such as SGS compared to a well-established baseline.

Section 6.1.1 outlines the methodology used. In Section 6.1.2 we focus on the effect, on Hugin as well as on SimpleSGS and OperatorSGS, of varying the $C / V$ ratio in BNs. In Section 6.1.3 we investigate additive gain using OPERATORSGS. 


\subsubsection{Synthetic Networks: Methodology}

In this section, we briefly discuss a paradigm for the generation of increasingly hard BNs for inference, namely the BPART algorithm [54, 61]. The BPART algorithm extends research on generating hard instances for the satisfiability problem [64], and we are exploiting the close relationship between computing an MPE and finding a satisfying truth assignment of a corresponding CNF formula [8, 72, 80]. Generating problem instances at random can result in very easy problems [64]. By carefully manipulating one or more BPART input parameters, one can construct BNs that existing tree clustering inference algorithms cannot handle due to an approximately exponential increase in clique tree size $[56,61]$. The main quantity we vary here is the ratio $C / V$, where $C$ and $V$ are BPART input parameters representing the number of leaf and root nodes in a $\mathrm{BN}$ respectively. The $C / V$-ratio is perhaps easiest to motivate using bipartite BNs for medical diagnosis, where $V$ is the number of diseases and $C$ is the number of symptoms. Clearly, it is interesting to understand how the speed of MPE computation varies when the ratio of symptoms to diseases is varied, or in other words as the $C / V$-ratio is varied. For the experiments reported here we set BPART's input parameters as follows, generating SAT-like BNs [61]: The CPT type of the root nodes was $Q=$ uniform; the CPT type of the non-root nodes was $F=$ or; the number of root nodes was $V=30$; the number of states per node was $S=2$; the number of parents per leaf node was $P=3$; and irregular BNs were created by setting $R=$ false. Here, "irregular" refers to the fact that each root node has an irregular or randomly varying number of children. We varied the number of leaf nodes while keeping $V=30$ constant, giving $C / V$-ratios ranging from $C / V=2.0$ to $C / V=3.4$.

Our main focus in the following is the comparison of the execution phase of HugIN with that of SGS (using approximately optimal parameter settings) when computing an MPE. Our methodology is to generate a number of BN instances according to the BPART construction, run SGS and HUGIN on these instances, and record statistics for the time it takes to compute an MPE. We do not report results for BNs that HugIN was not able to process, even in cases where SGS might have been able to find an MPE. For example, we did not experiment with as large SAT-like BNs as the SAT formulas used in earlier research [64], since HugIN was not able to process these large networks for interesting $C / V$-ratios. Consequently, our results do not include the region where $C / V \approx 4.25$, which is SAT's phase-transition region [64].

Even though these SAT-like BNs have a clear mapping to SAT instances, a few crucial differences make our BN experiments different from previous experiments in the SAT setting. Our BN data structures are different, since they are able to represent arbitrary CPTs, not just the logical true or false values that arise in pure logical inference. Along similar lines, our gain and utility computations are generalizations of their logical counterparts as discussed in Sections 3 and 4. They need to handle the general, real-valued case as encountered in CPTs, not just the special, integer-valued case of logical inference.

\subsubsection{Synthetic Networks: Hardness and the C/V Ratio}

The purpose of the experiments reported here was to compare the performance of SGS, both SimpleSGS and OperatorSGS, with that of Hugin as the $C / V$-ratio was 
varied. For each $C / V$ level, $100 \mathrm{BNs}$ were generated using the BPART algorithm and processed using HugIN and SGS.

Clique tree results for HugIN are summarized in Table 2; each row shows statistics for BPART instances. There are dramatic increases in total clique tree size as well as in the size of the largest clique as $C / V$ increases. The rapid growth of the total clique tree size with $C / V$ causes MPE computation times to grow rapidly as well. In Table 2, sample means and standard deviations of MPE computation times for Hugin are presented.

We also experimented with both variants of SGS, namely SIMPLESGS and OperATORSGS, with parameters set as follows: MAX-TRIES $=\infty, U=U_{A}, C=C_{G}$, and $U_{\min }=75$ (for $C=60$ ) to $U_{\min }=117$ (for $C=102$ ). MAX-FLIPS and $p_{N}$ were set to approximately optimal levels, varying with $C / V$. For both variants, an MPE was found for all problem instances.

In Fig. 9 the results from these experiments are shown along with exponential regression lines of the form $y=a e^{b x}$ where $a, b \in \mathbb{R}$ and $x=C / V$. For SimpleSGS and OPERATORSGS, each data point in the figure represent a total of 10,000 experiments. This is because there are $100 \mathrm{BNs}$ per $C / V$ level, and each SGS variant was executed 100 times for each BN. The exponential regression results in Fig. 9 show that the computation times of HUGIN and SGS increase with $x=C / V$ in a fashion that is wellapproximated by exponential curves. For Hugin, this confirms earlier results [56, 61]. For Hugin, we note also that the execution phase consists of several propagations when there is more than one MPE (Madsen 2003, personal communication). The existence of multiple MPEs for this $C / V$ range is reflected in the obvious difference

Table 2 Clique tree statistics (top half) and propagation times (bottom half) for HugIN on synthetic, bipartite Bayesian networks. Parameters characterizing these BNs are the number of root nodes $V$ and the number of leaf nodes $C$

\begin{tabular}{|c|c|c|c|c|c|c|c|c|c|c|}
\hline $\begin{array}{l}\mathrm{BNs} \\
\mathrm{C} / \mathrm{V}\end{array}$ & Mean & StDev & Median & Max & Min & Mean & StDev & Median & Max & Min \\
\hline & \multicolumn{5}{|c|}{ Total clique tree size (in 1000) } & \multicolumn{5}{|c|}{ Size of maximal clique (in 1000) } \\
\hline 2.0 & 201.7 & 95.17 & 172.1 & 513.3 & 53.34 & 70.94 & 44.49 & 65.54 & 262.1 & 16.38 \\
\hline 2.2 & 388.2 & 211.1 & 337.9 & 1,212 & 53.60 & 150.6 & 109.0 & 131.1 & 524.3 & 16.38 \\
\hline 2.4 & 573.8 & 288.3 & 504.5 & 1,628 & 131.8 & 238.6 & 157.1 & 262.1 & 1,049 & 65.54 \\
\hline 2.6 & 852.7 & 449.1 & 718.8 & 2,302 & 190.9 & 329.6 & 226.1 & 262.1 & 1,049 & 65.54 \\
\hline 2.8 & 1,285 & 789.0 & 1,158 & 5,463 & 278.5 & 503.3 & 356.3 & 524.3 & 2,097 & 131.1 \\
\hline 3.0 & 1,881 & 991.7 & 1,651 & 6,506 & 580.9 & 720.9 & 331.4 & 524.3 & 2,097 & 131.1 \\
\hline 3.2 & 2,559 & 1,241 & 2,337 & 6,468 & 607.6 & 1,077 & 669.5 & 1,049 & 4,194 & 262.1 \\
\hline 3.4 & 3,779 & 1,807 & 3,504 & 11,204 & 689.1 & 1,565 & 944.4 & 1,049 & 4,194 & 262.1 \\
\hline$\overline{\mathrm{BNs}}$ & Mean & StDev & Median & Max & Min & Mean & StDev & Median & Max & Min \\
\hline $\mathrm{C} / \mathrm{V}$ & \multicolumn{5}{|c|}{ Time, all propagations (s) } & \multicolumn{5}{|c|}{ Time, one propagation (s) } \\
\hline 2.0 & 1.119 & 0.5590 & 1.039 & 3.234 & 0.328 & 0.07038 & 0.03405 & 0.06491 & 0.1902 & 0.01726 \\
\hline 2.2 & 2.123 & 1.220 & 1.8 & 7.047 & 0.375 & 0.1404 & 0.08568 & 0.1236 & 0.5560 & 0.02344 \\
\hline 2.4 & 3.102 & 1.758 & 2.696 & 9.953 & 0.891 & 0.2291 & 0.1345 & 0.1952 & 0.7864 & 0.04689 \\
\hline 2.6 & 4.809 & 3.234 & 3.898 & 24.55 & 0.718 & 0.3655 & 0.2374 & 0.3015 & 1.444 & 0.1000 \\
\hline 2.8 & 6.349 & 4.249 & 5.258 & 25.64 & 1.234 & 0.5706 & 0.4164 & 0.5137 & 3.205 & 0.1198 \\
\hline 3.0 & 8.267 & 5.039 & 7.008 & 36.31 & 1.782 & 0.8618 & 0.4744 & 0.7428 & 3.026 & 0.2206 \\
\hline 3.2 & 12.76 & 8.332 & 10.79 & 41.91 & 2.875 & 1.293 & 0.6811 & 1.120 & 3.224 & 0.2756 \\
\hline 3.4 & 14.67 & 9.867 & 9.781 & 66.5 & 2.797 & 1.864 & 1.059 & 1.738 & 6.045 & 0.3108 \\
\hline
\end{tabular}




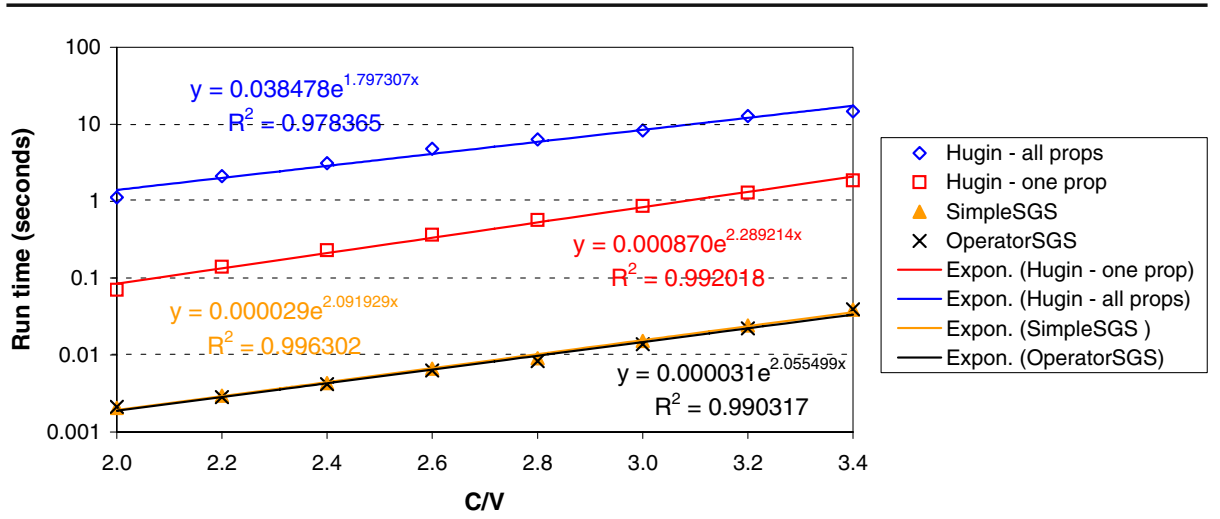

Fig. 9 A comparison of the performance of Hugin, SimpleSGS, and OperatorSGS as $C / V$ is varied. In general, HUGIN needs multiple clique tree propagations in order to find an MPE, hence results for one propagation ("HuGIN—one prop") as well as multiple propagations ("HugIN—all props") are shown. On average, both SimPLESGS and OpERATORSGS are more than one order of magnitude faster than one HUGIN propagation, and more than two orders of magnitude faster than all HUGIN propagations

between $y=0.03847 e^{1.797 x}$ (for all propagations) and $y=0.000870 e^{2.289 x}$ (for one propagation) in Fig. 9.

Figure 9 shows that both SimpleSGS and OperatorSGS clearly outperform Hugin. Specifically, for MPE computation we note that SimpleSGS and OperaTORSGS on average are more than two orders of magnitude faster than HugIN (the "all props" regression line in Fig. 9) on these BPART BNs where $2.0 \leq C / V \leq$ 3.4. Even if HugIN had always only needed one propagation (a best-case scenario reflected by the "one prop" regression line in Fig. 9) in order to compute an MPE, both SGS variants are on average well over one order of magnitude faster than Hugin for $2.0 \leq C / V \leq 3.4$. The run time curves of SimpleSGS and OperatorSGS in Fig. 9 are quite similar and in fact it is difficult to tell them apart. A potential disadvantage of OperatorSGS compared to SimpLESGS, namely slower speed due to computational overhead associated with operator selection and application, is in other words minimized in our current implementation.

\subsubsection{Synthetic Networks: Investigation of Additive Gain for Varying C/ V}

The goal of this set of experiments was to investigate different OperatorSGS variants. In particular, we wanted to investigate both multiplicative gain and additive gain in SAT-like BNs, generated using the BPART algorithm as discussed in Section 6.1.2. Since the additive measure is tailored to SAT-like BNs, one might expect SGS with $\Lambda_{S}=\left\{\left(\mathrm{AG}, p_{\mathrm{AG}}\right),\left(\mathrm{NU}, p_{\mathrm{NU}}\right)\right\}$ to outperform SGS with $\Lambda_{S}=\left\{\left(\mathrm{MG}, p_{\mathrm{MG}}\right)\right.$, $\left.\left(\mathrm{NU}, p_{\mathrm{NU}}\right)\right\}$ in this experiment. However, SGS with $\Lambda_{S}=\left\{\left(\mathrm{MG}, p_{\mathrm{MG}}\right),\left(\mathrm{NU}, p_{\mathrm{NU}}\right)\right\}$ is augmented with a "force state" heuristic which handles deterministic nodes, as shown in Fig. 3, and the question is how helpful this heuristic is compared to the additive measure. In this set of experiments, we again used $V=30$ root nodes, and considered $C=60$ and $C=102$. 
Results of the experiments are shown in Fig. 10. In the two panels to the left in the figure, we show results for OperatorSGS with $\Lambda_{S}=\left\{\left(\mathrm{AG}, p_{\mathrm{AG}}\right),\left(\mathrm{NU}, p_{\mathrm{NU}}\right)\right\}$, a portfolio with an additive operator AG. In the two panels to the right, we show results for OperatorSGS with $\Lambda_{S}=\left\{\left(\mathrm{MG}, p_{\mathrm{MG}}\right),\left(\mathrm{NU}, p_{\mathrm{NU}}\right)\right\}$, a portfolio with a multiplicative operator MG. Results for $C / V=2.0$ are displayed in the top two panels in Fig. 10, while the bottom two panels show results for $C / V=3.4$. In addition, we used three different values for $p=p_{\mathrm{NU}}$, and consequently for $p_{\mathrm{AG}}=1-$ $p_{\mathrm{NU}}$ and $p_{\mathrm{MG}}=1-p_{\mathrm{NU}}$ respectively, and varied MAX-FLIPS as shown on the $x$ axis in Fig. 10.

Our main observations with respect to these experimental results are as follows. For both $C / V=2.0$ and $C / V=3.4$, the additive approach (to the left in Fig. 10) is the winner over the multiplicative approach in the sense of having the parameter settings that minimizes the number of flips. In addition, the additive approach is more robust in the sense that there is good performance for a wider range of parameter values. This experiment provide an experimental rationale for the "artificial" additive approach, which is used in OperatorSGS with $\Lambda_{S}=\left\{\left(\mathrm{AG}, p_{\mathrm{AG}}\right),\left(\mathrm{NU}, p_{\mathrm{NU}}\right)\right\}$, compared to the multiplicative approach used in OperatorSGS with $\Lambda_{S}=\{(\mathrm{MG}$, $\left.\left.p_{\mathrm{MG}}\right),\left(\mathrm{NU}, p_{\mathrm{NU}}\right)\right\}$.
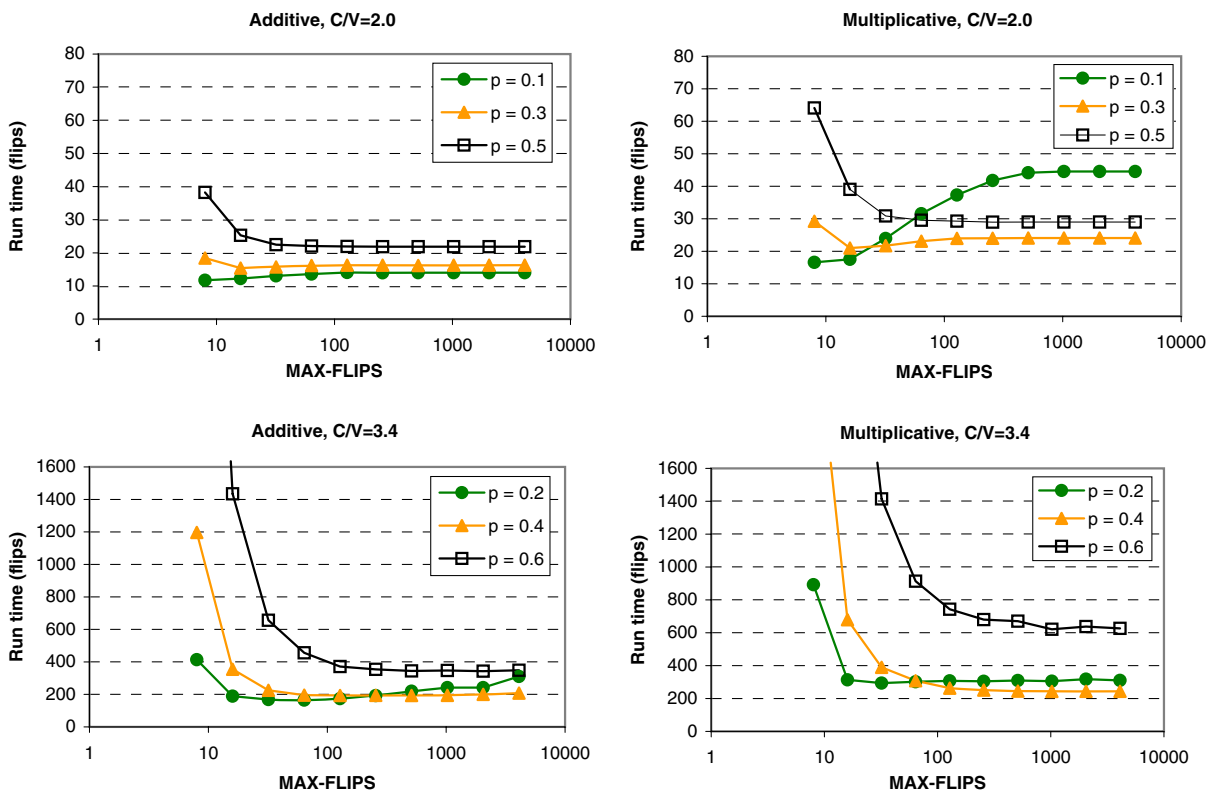

Fig. 10 Empirical results for synthetic BNs under different experimental conditions for OPERATORSGS. The additive search operator AG is used in the column to the left, the multiplicative search operator $\mathrm{MG}$ is used in the column to the right. The top row is for $C / V=2.0$ and the bottom row is for $C / V=3.4$. The restart parameter varies from MAX-FLIPS $=8$ to MAX-FLIPS $=4096$ as shown on the $x$-axis. The noise probability $p=p_{N}$ is varied according to the labels. Each data point represents the sample mean of 10,000 runs: 100 runs for each of $100 \mathrm{BNs}$ 
This section reports on SGS experiments using BNs from applications, and highlights the system's performance when using different initialization portfolios, different search portfolios, and different measures of gain. The OpERATORSGS algorithm was to a large extent motivated by our early work with these BNs, which unlike the synthetic instances investigated in Section 6.1 have no restriction on topology, number of states, number of parents, or CPTs.

In Section 6.2.1 we outline the methodology and briefly discuss the BNs used in experiments. Section 6.2.2 empirically compares SGS and HugIN. Section 6.2.3 covers the effect of using different search operators in OpERATORSGS, while Section 6.2.4 covers the effect of varying MAX-FLIPS in OpERATORSGS.

\subsubsection{Application Networks: Methodology}

The BNs investigated here, most of which are taken from Friedman's Bayesian Network Repository, are denoted Munin1, Munin2, Mildew, and Water. (At the time of this writing, the location of the Bayesian Network Repository is http://www. cs.huji.ac.il/labs/compbio/Repository/.) Given our emphasis on additive utility and gain derived from MAXSAT, we focus on BNs with a substantial number of zeros; see Table 3.

The Munin BNs, Munin1 and Munin2, are medical BNs from the field of electromyography. The Munin1 BN has, among the BNs we consider here, the largest total clique tree size and the slowest compile and per-propagation execution time. The Water BN models the biological processes of water purification, while Mildew is for making dosage recommendations regarding the amount of fungicide needed to fight mildew in wheat. For each of these BNs, statistics for the CPT values along with the number of nodes and the average number of states per node are presented in Table 3.

For these application BNs, we first ran Hugin. Following the methodology presented in Section 6.1.1, for each BN the resulting MPE probability $\operatorname{Pr}\left(\boldsymbol{x}^{*}\right)$ was then used as utility limit $U_{\min }=\operatorname{Pr}\left(\boldsymbol{x}^{*}\right)$ for SGS. This approach makes our comparison between algorithms more informative as discussed in Section 5.1.

Table 3 Information on Bayesian networks from applications. Parameters characterizing the BNs are the number of nodes $n$ and the average number of states per node. Conditional probability table values are also shown. These BNs have a large number of zeros in CPTs, thus for a large number of explanations $\boldsymbol{x}$ have $\operatorname{Pr}(\boldsymbol{x})=U_{B}(\boldsymbol{x})=0$. In these highly deterministic Bayesian networks, the additive approach turns out to reduce run time when used in stochastic local search

\begin{tabular}{lcclllllll}
\hline Bayesian & Nodes & States & \multicolumn{6}{l}{ Conditional probability table (CPT) values (in \%) } \\
\cline { 5 - 10 } network & \multicolumn{1}{c}{$n$} & avg. & 0 & $\left(0,10^{-3}\right]$ & $\left(10^{-3}, 10^{-2}\right]$ & $\left(10^{-2}, 10^{-1}\right]$ & $\left(10^{-1}, 1\right)$ & 1 \\
\hline Munin1 & 189 & 5.26 & 56.0 & 3.64 & 3.87 & 7.99 & 18.0 & 10.4 \\
Munin2 & 1003 & 5.36 & 55.5 & 4.74 & 5.08 & 9.46 & 17.4 & 7.74 \\
Mildew & 35 & 17.6 & 93.1 & 0.01 & 0.75 & 2.07 & 3.93 & 0.15 \\
Water & 32 & 3.63 & 51.7 & 0.04 & 1.4 & 12.7 & 31.8 & 2.35 \\
\hline
\end{tabular}




\subsubsection{Application Networks: SGS and HugIN}

The goal of the experiments we report on now is to compare the performance of OperatorSGS and Hugin using BNs from applications. Results for HugIN are shown in Table 4; the fill in weight triangulation heuristic was used. In Table 4, all HugIN timing results are averages of 30 experiments, except for Munin1 which is the average of five experiments.

Results for OpERATORSGS are shown in Table 5. We use approximately optimal parameter settings for OPERATORSGS. Reflecting our analytical result in Theorem 58, OPERATORSGS uses homogenous initialization portfolios with the forward simulation (FS) and forward dynamic programming (FDP) initialization operators here. Further experiments investigating the impact of varying the search portfolio and MAXFLIPS on the performance of OPERATORSGS can be found in Sections 6.2.3 and 6.2.4.

Comparing the results for HUGIN and OperatorSGS in Tables 4 and 5 respectively, we make the following observations. First, OPERATORSGS is highly competitive with Hugin for these BNs. Particularly impressive is the performance of OPERATORSGS for Munin1 and Water, where the computation time of OpERATORSGS (measured in seconds) is substantially better than HugIN's. For Munin1, one HugIN propagation takes 4,057.8 s while OperatorSGS computation time is 0.02537 s. For Water, one Hugin propagation takes 0.715 seconds while OperatorSGS computation time is $0.0625 \mathrm{~s}$. Second, for three of the BNs in Table 5, approximately optimal OPERATORSGS performance is obtained for additive probability $p_{A} \geq 0.9$, thus highlighting the power of our additive approach.

\subsubsection{Application Networks: Different Search Operators}

In this set of experiments with OperatorSGS, we investigated different search operators by varying the search portfolio $\Lambda_{S}$. The purpose of these experiments was to investigate the effect of varying additive probability $p_{A}$ and noise probability $p_{N}$. Appropriate use of noise has been found to be a powerful way to improve SLS performance [20, 31, 34, 53, 57, 76, 77]; however we know of no previous experiments where the effect of varying additive probability $p_{A}$ is systematically investigated. To do so, we considered the following search portfolio.

Definition 61 (Search portfolio) The search portfolio $\Lambda_{S}\left(p_{N}, p_{A}\right)$ is parametrized by noise probability $p_{N}$ and additive probability $p_{A}$ and is defined as

$$
\begin{gathered}
\Lambda_{S}\left(p_{N}, p_{A}\right)=\left\{\left(\mathrm{AN}, p_{A} p_{N}\right),\left(\mathrm{AG}, p_{A}\left(1-p_{N}\right)\right),\left(\mathrm{MN},\left(1-p_{A}\right) p_{N}\right),\right. \\
\left.\left(\mathrm{MG},\left(1-p_{N}\right)\left(1-p_{A}\right)\right)\right\} .
\end{gathered}
$$

Table 4 Performance of Hugin on application Bayesian networks. These are clique tree statistics, and the results in the Total column are for computing an MPE. The Compile and Execute columns

\begin{tabular}{|c|c|c|c|c|c|c|c|}
\hline \multirow[t]{2}{*}{$\mathrm{BN}$} & \multicolumn{3}{|c|}{ Clique tree statistics } & \multicolumn{4}{|c|}{ Execution times (s) } \\
\hline & Sum & Max & Median & Compile & Propagate & Execute & Total \\
\hline Munin1 & $384,620,599$ & $288,000,000$ & 300 & $6,058.2$ & $4,057.8$ & $4,057.8$ & $10,114.8$ \\
\hline Munin2 & $4,861,824$ & 504,000 & 320 & 2.089 & 0.815 & 2.445 & 4.535 \\
\hline Mildew & $9,566,232$ & $4,372,480$ & 8,600 & 1.0671 & 0.821 & 3.284 & 4.352 \\
\hline Water & $3,657,180$ & $1,769,472$ & 2,187 & 0.616 & 0.715 & 0.715 & 1.330 \\
\hline
\end{tabular}
give the time taken in each of these two phases 
Table 5 Performance of OPERATORSGS on application Bayesian networks. The values of approximately optimal input parameters, including initialization and search operators, as well as results for computing an MPE are shown. Note how $p_{A}$, or additive probability, is non-zero in all cases

\begin{tabular}{|c|c|c|c|c|c|c|}
\hline \multirow[t]{2}{*}{$\mathrm{BN}$} & \multicolumn{4}{|c|}{ Input parameters } & \multicolumn{2}{|c|}{ Execution times } \\
\hline & Initialization & Search & MAX-FLIPS & $p_{A}$ & Time (s) & Flips \\
\hline Munin1 & $p_{\mathrm{FS}}=1.0$ & $\begin{array}{r}p_{\mathrm{MN}}=0.05, p_{\mathrm{MG}}=0.05 \\
p_{\mathrm{AG}}=0.45, p_{\mathrm{AN}}=0.45\end{array}$ & 30 & 0.9 & 0.02537 & 25.37 \\
\hline Munin2 & $p_{\mathrm{FS}}=1.0$ & $p_{\mathrm{AN}}=0.5, p_{\mathrm{AG}}=0.5$ & 70 & 1.0 & 1.385 & 245.5 \\
\hline Mildew & $p_{\mathrm{FDP}}=1.0$ & $\begin{array}{l}p_{\mathrm{MN}}=0.336, p_{\mathrm{MG}}=0.504 \\
p_{\mathrm{AN}}=0.064, p_{\mathrm{AG}}=0.096\end{array}$ & 200 & 0.16 & 0.2404 & 193.3 \\
\hline Water & $p_{\mathrm{FS}}=1.0$ & $p_{\mathrm{AN}}=0.1, p_{\mathrm{AG}}=0.9$ & 10 & 1.0 & 0.00625 & 61.71 \\
\hline
\end{tabular}

By introducing $\Lambda_{S}\left(p_{N}, p_{A}\right)$, we reduce the three-dimensional problem of varying probabilities in the portfolio $\Lambda_{S}\left(p_{\mathrm{AN}}, p_{\mathrm{AG}}, p_{\mathrm{MN}}\right)$ from Definition 46 into a twodimensional problem of varying probabilities $p_{N}$ and $p_{A}$. Reflecting Theorem 58, a $\mathrm{BN}$-specific optimal initialization algorithm-either forward simulation [28] or a randomized variant of the Viterbi algorithm $[62,82]$ — was used in $\Lambda_{I}$ for each application BN. Forward simulation was used for Munin1, Munin2, and Water. Mildew was initialized using the forward variant of the randomized Viterbi algorithm. BNspecific values for MAX-FLIPS were also used: MAX-FLIPS $=30$ for Munin1, MAX-FLIPS $=100$ for Munin2, MAX-FLIPS $=10$ for Water, and MAX-FLIPS $=$ 200 for Mildew.

For each application $\mathrm{BN}$, the probability of using an additive operator, $p_{A}$, was varied from $p_{A}=0$ to $p_{A}=1$ in increments of $\Delta p_{A}=0.1$. Noise probability $p_{N}$ was varied from $p_{N}=0.1$ to $p_{N}=0.9$ in increments of $\Delta p_{N}=0.2$. We call these and similar run time curves generated by varying the probability $p_{A}$ "additive response curves", since they are similar to noise response curves generated by varying the probability $p_{N}[31,53]$. Results, in the form of sample means and piecewise linear approximations for $\hat{r}\left(p_{A}\right)$, are reported in Fig. 11. Each data point represents the mean of 1,000 experiments.

In Fig. 11, the case $p_{N}=p_{G}=0.5$ represents the situation where greedy and noise search operators are applied with equal probability. Further, at $p_{A}=0$ only the operators $\mathrm{MN}$ and $\mathrm{MG}$ are applied, while at $p_{A}=1$ only $\mathrm{AN}$ and $\mathrm{AG}$ are used. Previous experimental results are at $p_{A}=0$ if they only use multiplicative gain and not additive gain in their search heuristics; we will shortly see the disadvantage of this traditional approach.

Some of the main points found in Fig. 11 are as follows. One main novel result is that varying $p_{A}$ has, in many cases, a striking impact on SLS run time. Specifically, by using $p_{A}=0$ as is employed in most traditional SLS approaches to BN inference, one may obtain far from optimal performance. The impact depends on the particular $\mathrm{BN}$ and also varies dramatically with noise level $p_{N}$.

In Fig. 11, the shapes of the additive response curves for Water, Munin1, and Munin2 are quite similar: Optimal levels of $p_{A}, p_{A}^{*}$, appear to be found at high values of $p_{A}$, and for such values the impact of noise is relatively minor. Combining high noise probability $p_{N}$ and low additive probability $p_{A}$ has, on the other hand, a very negative impact on run time. Examples of this can be found for $p_{N}=0.7$ and $p_{A}=$ 

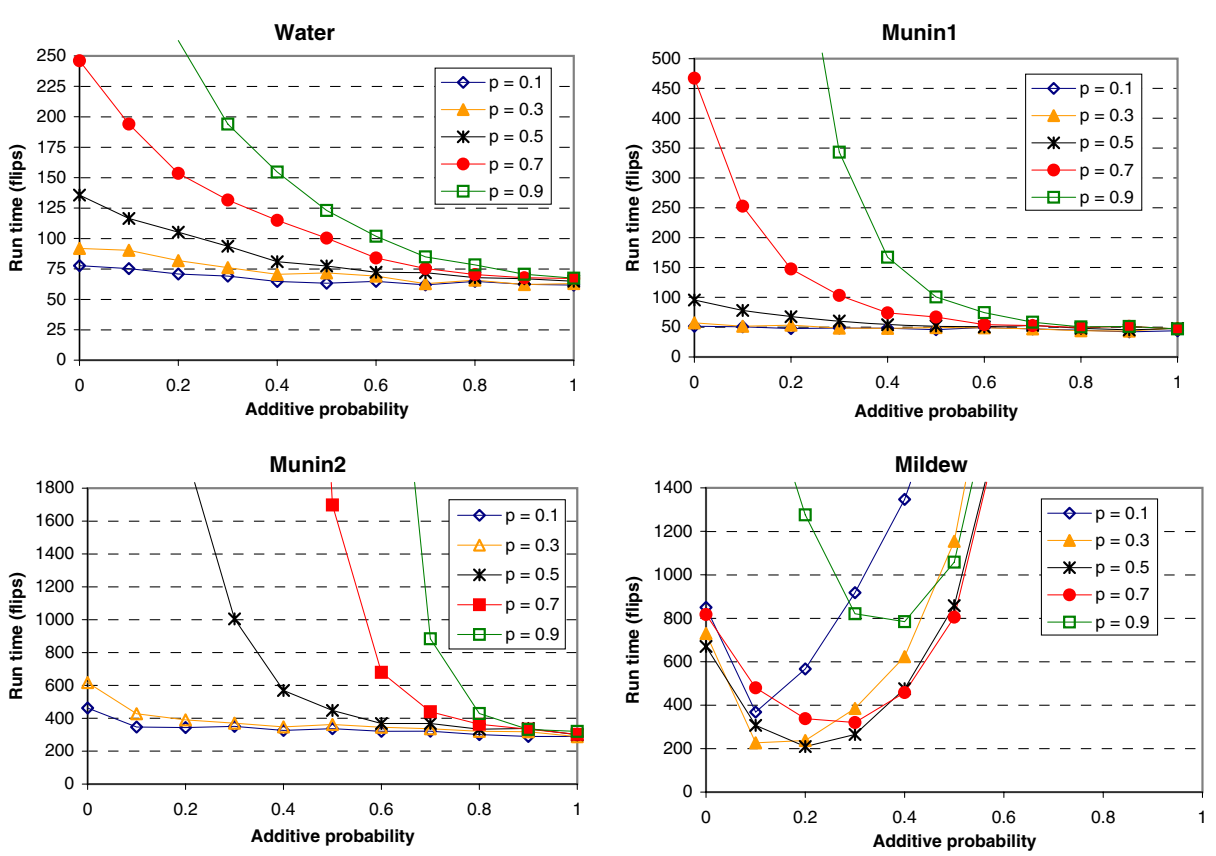

Fig. 11 Empirical results for OperatorSGS for the BNs Munin1, Munin2, Water, and Mildew under different experimental conditions. The additive probability $p_{A}$, or the probability of applying an additive search operator, varies from $p_{A}=0$ to $p_{A}=1$ as shown on the $x$-axis. The noise probability $p=p_{N}$ is varied from $p_{N}=0.1$ to $p_{N}=0.9$ according to the labels. Each data point represents the sample mean of 1,000 runs. Clearly, varying $p_{A}$ and $p_{N}$ has a significant impact on run time

0.1 in Fig. 11 for Water, Munin1, and Munin2. Robust OperatorSGS performance, with respect to variations in $p_{N}$, is ensured by using a high value for $p_{A}$.

The shapes of the OperatorSGS run time curves for Mildew are somewhat different than those for Water, Munin1, and Munin2. Here we find, in all cases, monotonic decreases in run times for small values of $p_{A}$ and monotonic increases in run times for large values of $p_{A}$. These convex empirical curves suggest an optimal value for $p_{A}^{*}$ that is increasing with $p_{N}$. A relatively high level of noise improves the run time on Mildew; $p_{N}=0.5$ gives best results in these experiments, with an average run time of 209.8 flips at $\hat{p}_{A}^{*}=0.2$.

\subsubsection{Application Networks: Impact of Restart}

In this section we investigate the impact, on OPERATORSGS performance, of varying the restart parameter MAX-FLIPS along with $p_{A}$ and $p_{N}$. We experiment with Mildew since it has the most challenging (and interesting) additive response curve in Fig. 11. Similar to in Section 6.2.3, we employ the search portfolio $\Lambda_{S}\left(p_{N}, p_{A}\right)$. In order to further investigate the region where minimal average run time occurred in Fig. 11 , we varied additive probability $p_{A}$ from $p_{A}=0.1$ to $p_{A}=0.3$ in increments of $\Delta p_{A}=0.02$. Noise probability $p_{N}$ was varied from $p_{N}=0.3$ to $p_{N}=0.6$ in increments of $\Delta p_{N}=0.1$. 

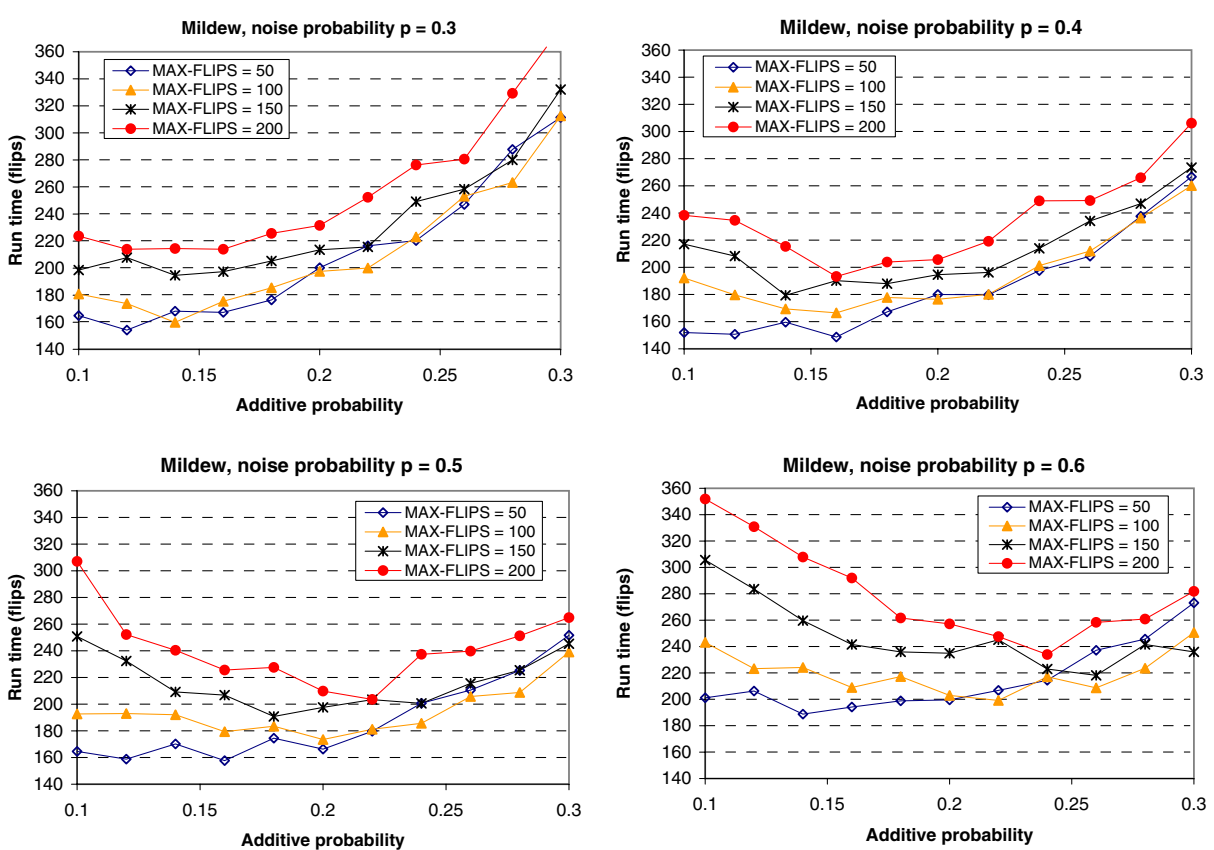

Fig. 12 Empirical results for the Mildew BN under different experimental conditions for OPERATORSGS. The probability $p_{A}$ of applying an additive search operator, varying from $p_{A}=0.1$ to $p_{A}=0.3$, is shown on the $x$-axis. The noise probability $p=p_{N}$ is systematically varied from $p_{N}=0.3$ (top left) to $p_{N}=0.6$ (bottom right), and the restart parameter MAX-FLIPS is varied according to the labels. Each data point represents the sample mean of 1,000 runs. Clearly, varying $p_{A}, p_{N}$, and MAX-FLIPS has a significant impact on run time

Results, in the form of sample means and piecewise linear approximations for $\hat{r}\left(p_{A}\right)$, are reported in Fig. 12. Each data point represents the mean of 1,000 runs. In each panel of Fig. 12, varying MAX-FLIPS has a substantial impact. Generally, MAX-FLIPS $=50$ gives the fastest run time. Further, careful optimization of $p_{A}$, $p_{N}$, and MAX-FLIPS gives significant benefit, and the approximate performance optimum is at $p_{A}=0.17, p_{N}=0.4$, and MAX-FLIPS $=50$.

\section{Related Work and Discussion}

The problem of computing a most probable explanation (MPE) in Bayesian networks has been addressed using exact algorithms [2, 37, 45, 47, 70, 79, 85] as well as inexact algorithms [39, 41, 48, 54, 60]. In addition, there is related work on SAT and MAXSAT [27, 35, 53, 75, 77, 78] as well as on weighted MAXSAT $[10,19,29,42,44,46,66,74,83]$. Since a comprehensive literature review is well beyond the scope of this article, we only discuss the previous research most closely related to our work in the rest of this section. 


\subsection{Portfolio Algorithms}

Our work is related to research on hybrid or portfolio algorithms [25, 26, 38, 84]. Huberman, Lukose, and Hogg investigate hard computational problems and how heuristic algorithms, often randomized, have been developed to solve such problems [38]. Their main emphasis is on portfolios of independent Las Vegas algorithms, where run times can be described using probability distributions, and consequently one can form expectation and variance (or standard deviation). By considering the 2-dimensional space spanned by expectation and standard deviation, an efficient frontier can be formed, similar to what is done in economics, based on varying the fractions of CPU-time allocated to different Las Vegas algorithms in a portfolio. Empirically, the NP-hard problem of graph coloring was studied using the Brelaz heuristic. By taking a portfolio approach, it was found that expected performance could be increased by $30 \%$ while also reducing risk (or standard deviation) [38]. While the main emphasis of Huberman, Lukose, and Hogg is on completely independent algorithms, they also briefly discuss cooperating algorithms which is our main focus in SGS.

Gomes and Selman note that performance profiles vary dramatically among different algorithms over different problem instances [25]. In response, they investigate portfolios of algorithms, and find that it can be beneficial to combine algorithms with high run time variance in portfolios. Gomes and Selman consider one or more Las Vegas algorithms running independently and in parallel on multiple processors. They investigate constraint satisfaction and mixed integer programming problems empirically, and conclude that optimal portfolio design strongly depends on details of the run time distribution.

Lagoudakis and Littman formalize algorithm selection as a Markov decision process, and investigate a reinforcement learning approach to learning the value function [43]. Algorithms are selected from the portfolio in a cooperative, not independent, manner. Experimentally, they obtain promising results on algorithms for sorting and order statistics selection. Xu et al. integrate a learning approach with SAT algorithm portfolios [84]. All algorithms in the portfolio execute all problem instances in the training set in order to learn empirical hardness models. Learning is performed using features that characterize the problem instances, and learned ridge regression models are used to predict run times for individual problem instances. During test, a problem instance is solved by the SAT algorithm predicted to be fastest, hence there is no cooperation between algorithms. Using this approach, strong results in the 2007 SAT competition have been reported [84].

\subsection{Stochastic Local Search for SAT Computation}

We distinguish between local search algorithms that rely on the use of a history or memory (of search)-and those that do not. For example, tabu-search [24, 67] and guided local search $[39,63,66]$ are both meta-heuristics that introduce histories into local search. Roughly speaking, guided local search modifies the objective function during search as a tactic to escape states that are local minima, while tabu search dynamically maintains a list of search space states that it does not immediately revisit. While the use of history has been found to be powerful, it also significantly complicates analysis, and we here emphasize algorithms that do not rely on histories. 
We first consider related research on stochastic local search for solving the satisfiability problem (SAT) $[27,35,53,75,77,78]$. An early contribution was the GSAT algorithm, which searches for a satisfying truth assignment in a CNF formula [78]. GSAT is controlled by the number of local search steps before a restart, MAXFLIPS, and the number of restarts, MAX-TRIES [78]. A major contribution of GSAT was its "sideways moves"; the algorithm continues to flip variables even when the number of satisfied clauses stays the same. Experiments showed that GSAT outperformed the Davis-Putnam (DP) algorithm on synthetic instances [64], and strong performance on other problems including graph coloring, the $\mathrm{N}$-queens problem, and Boolean induction were also reported [78].

The GSAT algorithm has been extended in a number of directions (see [35] for a summary). For instance, the three heuristic techniques of clause weighing, averaging in of previous near solution, and (mixed) random walk have all been found to improve the performance of basic GSAT [76]. Mixed random walk, which is also known as WALKSAT [53, 77], combines random walk and greedy local search and is more focused than random noise in that it applies noise only in variables that occur in unsatisfied clauses. The main conclusion of an empirical evaluation was that mixed random walk is superior to simulated annealing and random noise in a wide range of cases [77]. Further flip selection strategy improvements-such as WSAT-G [53], WSAT-B [53], Novelty [53], Novelty+, and DLM [75] — have followed, along with progress in noise adaptation $[20,31,53]$.

\subsection{Stochastic Local Search for MPE and MAP Computation}

We now discuss related research using stochastic search techniques to compute MPE or MAP in Bayesian networks [41, 48, 54, 60, 67, 68, 71]. Pearl argued early on that stochastic simulation, also known as Gibbs sampling, can be used for computing the MPE although the algorithm was primarily intended for belief updating [71, p. 216, p. 262]. In stochastic simulation, evidence nodes are clamped, and then random samples are created by randomly picking among a node's states based on their probabilities as given the node's Markov blanket.

An early investigation of MPE computation by means of stochastic search techniques considers iterative local search (ILS), simulated annealing (SA), and genetic search (GS) [48]. ILS is a probabilistic hill-climbing that combines next-ascent and random-mutation hill climbing. ILS stops on (local) maxima, however since it iterates, it typically finds many maxima, one of which might be an MPE. For the empirical study of these algorithms a bipartite BN version of the QMR medical knowledge base, QMR-DT, was used. Experiments with ILS, SA, and GS showed that they converged, in many cases, to an MPE. In terms of computational speed, ILS was found to be significantly faster than SA which was significantly faster than GS. However, SA was more accurate than ILS and GS. It is speculated that the weaker performance of ILS was due to the local search getting trapped in the plethora of local maxima [48].

Kask and Dechter empirically investigated MPE computation using stochastic simulation [41], and concluded that it does not perform as well as a greedy approach or as an approach where stochastic simulation and greedy search is combined. In addition, they found that augmenting the approach with the mini-bucket algorithm for initialization was important, which is in line with our results. We note that both 
stochastic simulation and greedy search are operators in OPERATORSGS, such that combining greedy search and stochastic simulation, as well as additional algorithms, within the SGS framework is easy. For the purpose of computing MAP, which generalizes MPE, Park and Darwiche investigated a total of 11 SLS algorithms and found that they performed very well, in particular when MPE-based initialization was used [68].

\subsection{Weighted MAXSAT and Bayesian Networks}

Recently, much progress has been made in developing solvers for the weighted MAXSAT problem [19, 29, 42, 44, 46, 83]. An early algorithm was WeIGHTEDWALKSAT [42], a generalization of WALKSAT. It was emphasized how the weighted MAXSAT problems could encode hard and soft constraints in discrete optimization problems, including NP-complete problems such as network Steiner tree problems. Network Steiner tree problems are concerned with finding paths in graphs. Using an approximate encoding of Steiner tree problems, for which much of the numeric information can be represented using clause weights, WEIGHTEDWALKSAT was shown to produce strong empirical results [42]. The GRASP algorithm also solves weighted MAXSAT using local search. Specifically, GRASP uses greedy randomized adaptive search, computing locally optimal solutions and using path relinking techniques to improve search [19].

The weighted MAXSAT problem can also be solved using exact (complete) algorithms, often based on the DP [13] and DPLL [12] algorithms. The MAXSolver uses heuristic strategies for DPLL, giving strong performance in experiments with random problems instances as well as problems instances from applications [83]. Other weighted MAXSAT solvers, for which details are beyond the scope of this article, include the MaxSATz [46], MiniMaxSAT [29], and MaxDPLL [44] solvers.

There is a close connection between weighted model counting and MPE computation $[10,66,74]$, and the use of weighted MAXSAT solvers to solve the MPE problem and the use of MPE solvers to solve the weighted MAXSAT problem has been investigated [66, 74]. Park encodes the MPE problem as a weighted MAXSAT problem, uses weighted model counting algorithms to compute answers to probabilistic queries, and finds that the incomplete guided local search (GLS) algorithm performs very well [66].

\subsection{Other Related Work}

Other related work includes exact $\mathrm{BN}$ algorithms such as clique (or join) tree propagation $[2,40,45,79]$, conditioning [9, 70], variable elimination [15, 47, 85], arithmetic circuit evaluation [6,11], and AND/OR search [17]. The compilation paradigm, which underlies clique tree propagation and arithmetic circuit evaluation, is wellsuited to resource-bounded and real-time settings [55, 65], and has found application in sensor validation and diagnosis of electrical power systems in aerospace vehicles $[58,59]$. Recently, a connection between BNs and multi-linear functions has been made $[10,11]$, supporting the compilation of BNs into arithmetic circuits $[6,10,11]$. The compilation of BNs into arithmetic circuits may rely on encoding of a BN into a CNF formula [10], which has been shown to take advantage of determinism [4] as well as other local structure in BNs [5]. Chavira and Darwiche encode a BN 
in the form of a weighted CNF theory, and investigate the effect of search versus compilation; different encodings; and local structure and evidence [5, 7].

\subsection{Discussion and Comparison}

We now discuss how our SGS approach is similar to and different from related research, and also how OperatorSGS and SimpleSGS compare. SimpleSGS was primarily inspired by previous seminal research on stochastic local search for SAT, in particular the GSAT and WALKSAT algorithms [77, 78]. SGS is also related to ILS and SLS, and extends ILS by applying to a wider range of $\mathrm{BN}$ topologies, using the additive measure, and using more advanced initialization operators before local search commences. We also note that the random-mutation hill climbing of ILS gives an effect similar to that of SGS noise operators. SLS [41], which was developed independently of SGS, shares several characteristics with SGS, such as stochastic steps and initialization that goes beyond initialization uniformly at random. However, there are several important differences between SGS and SLS, including the initialization algorithms, the fact that SGS can use different measures of gain (including additive gain), as well as the fact that SGS has an operator-based variant OPERATORSGS.

OpERATORSGS makes a stronger distinction between utility and gain than SIMPLESGS and other SLS algorithms. A key point is that each compound search operator has an associated gain, which is unrelated to how an explanation's overall utility is computed using $U$. So, for example, some search operators in $\Lambda_{S}$ can use additive gain $\Delta U_{A}$, while the overall utility of an explanation can be computed using multiplicative utility $U_{M}$. This provides greater flexibility than what is present in SimpLESGS and other SLS algorithms. Operators that use the additive measure are optimized for the special but often occurring case of deterministic nodes, while operators using the multiplicative measure are optimized for the general case of probabilistic nodes. SIMPLESGS as well as other SLS algorithms can not use both measures in the same invocation of the algorithm, while OperatorSGS can. Since many application BNs contain both probabilistic nodes and deterministic nodes, it turns out that using $\Delta U_{A}$ and $\Delta U_{M}$ in combination gives, in many cases, faster computation of MPEs using SGS. See Section 6.2 for supporting experiments with application BNs.

OperatorSGS can simulate SimpleSGS in the following manner: One can regard the noise and hill-climbing steps of SimpLESGS as two operators chosen from a stochastic search portfolio $\Lambda_{S}$ of size two, where one search operator is a greedy operator such as AG, the other is a stochastic operator such as $\mathrm{NU}: \Lambda_{S}=\{(\mathrm{NU}$, $\left.\left.p_{N}\right),\left(\mathrm{AG}, 1-p_{N}\right)\right\}$. And we can regard SimpleSGS initialization as an initialization operator chosen from an OPERATORSGS stochastic initialization portfolio $\Lambda_{I}$ of size one: $\Lambda_{I}=\{(\mathrm{UN}, 1)\}$. The advantage of OPERATORSGS compared to standard SGS is generality and flexibility, which typically leads, as shown in experiments in Sections 6.1 and 6.2, to better performance. Further, as detailed in Section 6.1, the speed of OperatorSGS and SimpleSGS is essentially the same on SAT-like BNs.

Previous portfolio-based approaches have emphasized running multiple heuristics independently, either on the same computer or independently on multiple computers $[25,38]$. Alternatively, they have picked, for a particular problem instance, the best algorithm among a portfolio of algorithms [84]. In comparison, there is in 
OpERATORSGS a cooperation between heuristics (similar to [43]), in the sense that initialization and search operators work on the same explanation $\boldsymbol{x}$ to compute an MPE estimate $\hat{\boldsymbol{x}}^{*}$. There is currently no learning in OPERATORSGS, unlike in some related work [36, 43, 73, 84]. Finally, we note that our Las Vegas analysis and experimental approach is different from most previous efforts in several ways. First, we emphasize finding an MPE, and not just getting close to it, and therefore we compare with an exact method, the Hugin clique tree clustering algorithm. Second, our synthetic networks are constructed in a systematic way, generating instances of varying hardness. This allows us to experimentally show that SGS can outperform HugIN on synthetic BN of increasing hardness.

Comparing SLS algorithms with a broader range of algorithms including those for weighted MAXSAT [10, 19, 29, 42, 44, 46, 66, 74, 83], we find that two broad approaches are emerging: In the encoding approach, instances of one problem (say, MPE) can be encoded as another (say, weighted MAXSAT). A current view is that MPE and weighted MAXSAT are complementary, where an instance of one problem can be encoded as an instance of the other problem, with some loss of effectiveness [74]. In the generalization approach, on the other hand, one or more existing algorithms or techniques are generalized to handle a wider range of problems. For instance, one may generalize from MAXSAT to weighted MAXSAT (see $[42,44]$ ) or from MAXSAT to MPE (as in [57] and in this article). The two approaches, encoding and generalization, both have their pros and cons. In the encoding approach, there is a separate encoding or compilation step that can be performed off-line. This step can thus be amortized over a potentially large number of queries to the same problem instance. A disadvantage is that encoding may lead to a blow-up in problem instance size. Understandability and simplicity are, on the other hand, facilitated by the generalization approach; disadvantages include the lack of an off-line step and ability to take advantage of special problem structure. It appears that both approaches merit investigation, and in this paper we generally are taking a generalization approach.

\section{Conclusion and Future Work}

Stochastic local search has proven to be powerful algorithms for finding satisfying assignments in satisfiability (SAT) instances [27, 35, 53, 75, 77, 78]. In this article, we discuss the generalization of stochastic local search from the logical case of SAT to the probabilistic case of Bayesian networks (BNs). While SLS techniques for MPE and MAP computation are well established [41, 48, 60, 67, 68], we present here several novel techniques and results centering around search and initialization portfolios as well as an additive measure of utility and gain. These techniques have been incorporated into the SGS stochastic local search approach, which heuristically computes a most probable explanation (MPE) in a Bayesian network. SGS combines greedy search, stochastic search, and stochastic initialization algorithms. We have presented two SGS algorithms, namely simple SGS (SIMPLESGS) and operator-based SGS (OperatorSGS); the latter is more flexible and general than the former due to its initialization and search portfolios. The initialization and search portfolios of OPERATORSGS contain operators, which are algorithms for initialization or search respectively, along with probabilities that control their selection and execution. The 
OPERATORSGS algorithm, for which we provide both theoretical and experimental results, is closely related to previous research on SLS algorithms for MPE and MAP computation $[41,67,68]$ as well as to work on portfolio (or hybrid) algorithms for other computational problems [25, 26, 38, 39, 60, 84].

We carefully formalized the concepts of SLS utility and gain in the context of MPE computation, and emphasized our additive approach. The additive measure, for which we provided several new results, is a generalization of GSAT's and WALKSAT's utility and gain measures to the probabilistic setting. Application BNs often have many deterministic nodes, and the additive measure turned out to be very powerful in such BNs. This measure has, to our knowledge, not been extensively studied in previous research on MPE computation using stochastic local search, and we also investigated how it relates to traditional multiplicative gain as used in other SLS algorithms for MPE. In addition, we introduced a novel Markov chain model, augmented random walk, where the behavior of OPERATORSGS's initialization and search operators is explicitly represented as Markov chain states. We also showed that the optimal OperatorSGS initialization portfolio is homogenous.

Two sets of experiments have been conducted. We have shown that SGS outperforms HugIN on hard BNs constructed from satisfiability instances, and also outperforms HugIN on application BNs with a high degree of determinism. In one set of experiments, we have utilized an approach to constructing synthetic Bayesian networks of varying hardness [61]. This approach helps in developing an understanding of the suitability of different algorithms for different classes of BNs. In these experiments, we found that SGS can outperform HuGIN by well over one order of magnitude, and in particular that SGS consistently performed one or more orders of magnitude better than the state-of-the-art exact algorithm HugIN as the $C / V$ ratio was varied. In addition, we found that the additive measure $U_{A}$ gave better performance than the multiplicative measure $U_{M}$ in partly deterministic, SAT-like networks.

Experiments with OperatorSGS on application BNs have also been done. We have found that the algorithm is quite effective on these networks too, and performs comparably to Hugin. Key factors in the success of OperatorSGS are the initialization operators, the additive measure, and the approach of using a portfolio of operators for computation. OPERATORSGS's varying approximately optimal parameter values, including values for the selection probabilities of the initialization and search operators, highlights the importance of the portfolio approach to stochastic local search.

Areas for current and future work include the following. First, the important role of portfolios and different measures of gain, especially in application networks, highlights the opportunity of adaptively tuning the probabilities (similar to noise adaptation $[20,31,53])$ of portfolio operators when computing MPE or MAP for a given $\mathrm{BN}$. While our focus here has not been on adaptation or learning during search, we believe that our framework and results can also enable innovations in these areas, thereby further enhancing portfolio-based stochastic local search algorithms. Both analytical and experimental work on portfolio adaptation would be of great interest. Second, in local search, an essential question is how to escape from local minima. In this article, our answer has been the use of noise, which is a local escape mechanism. Another approach is to use crossover from genetic algorithms. In the MPE or MAP context, crossover would take place between two explanations, thus providing a 
more global escape mechanism than noise. More generally, there is potential for additional hybridization, for example combining stochastic local search with clique tree clustering. Third, additional investigation of when to terminate, other than by using a Las Vegas approach or time limits, is needed in order to increase the easy-ofuse of the SGS approach in applications.

Acknowledgements This material is based, in part, upon work by Ole J. Mengshoel supported by NASA awards NCC2-1426 and NNA07BB97C as well as NSF grants CCF-0937044 and ECCS0931978. Ole J. Mengshoel and David C. Wilkins gratefully acknowledge support in part by ONR grant N00014-95-1-0749, ARL grant DAAL01-96-2-0003, and NRL grant N00014-97-C-2061. Dan Roth gratefully acknowledges the support of NSF grants IIS-9801638 and SBR-987345.

David Fried and Song Han are acknowledged for their co-development of the Raven software, used in experimental work reported here. Comments from the anonymous reviewers, which helped improve the article, are also acknowledged.

\section{References}

1. Abdelbar, A.M., Hedetnieme, S.M.: Approximating MAPs for belief networks is NP-hard and other theorems. Artif. Intell. 102, 21-38 (1998)

2. Andersen, S.K., Olesen, K.G., Jensen, F.V., Jensen, F.: HUGIN—a shell for building Bayesian belief universes for expert systems. In: Proceedings of the Eleventh International Joint Conference on Artificial Intelligence, vol. 2, pp. 1080-1085. Detroit, MI (1989)

3. Arnborg, S.: Efficient algorithms for combinatorial problems on graphs with bounded decomposability-a survey. BIT 25, 2-23, (1985)

4. Chavira, M., Darwiche, A.: Compiling Bayesian networks with local structure. In: Proceedings of the 19th International Joint Conference on Artificial Intelligence (IJCAI), pp. 1306-1312 (2005)

5. Chavira, M., Darwiche, A.: Encoding CNFs to empower component analysis. In: Proceedings of the 9th International Conference on Theory and Applications of Satisfiability Testing (SAT), vol. 4121, pp. 61-74. Lecture Notes in Computer Science, Springer, Berlin (2006)

6. Chavira, M., Darwiche, A.: Compiling Bayesian networks using variable elimination. In Proceedings of the Twentieth International Joint Conference on Artificial Intelligence (IJCAI-07), pp. 2443-2449. Hyderabad, India (2007)

7. Chavira, M., Darwiche, A.: On probabilistic inference by weighted model counting. Artif. Intell. 172(6-7), 772-799 (2008)

8. Cooper, F.G.: The computational complexity of probabilistic inference using Bayesian belief networks. Artif. Intell. 42, 393-405 (1990)

9. Darwiche, A.: Recursive conditioning. Artif. Intell. 126(1-2), 5-41 (2001)

10. Darwiche, A.: A logical approach to factoring belief networks. In: Proceedings of the Eight International Conference on Principles and Knowledge Representation and Reasoning (KR-02), pp. 409-420 (2002)

11. Darwiche, A.: A differential approach to inference in Bayesian networks. J. ACM 50(3), 280-305 (2003)

12. Davis, M., Logemann, G., Loveland, D.: A machine program for theorem-proving. Commun. ACM 5(7), 394-397 (1962)

13. Davis, M., Putnam, H.: A computing procedure for quantification theory. J. ACM 7(3), 201-215 (1960)

14. Dawid, A.P.: Applications of a general propagation algorithm for probabilistic expert systems. Stat. Comput. 2, 25-36 (1992)

15. Dechter, R.: Bucket elimination: a unifying framework for reasoning. Artif. Intell. 113(1-2), 41-85 (1999)

16. Dechter, R., El Fattah, Y.: Topological parameters for time-space tradeoff. Artif. Intell. 125 (1-2), 93-118 (2001)

17. Dechter, R., Mateescu, R.: AND/OR search spaces for graphical models. Artif. Intell. 171(2-3), 73-106 (2007)

18. Dechter, R., Pearl, J.: Network-based heuristics for constraint satisfaction problems. Artif. Intell. 34(1), 1-38 (1987) 
19. Festa, P., Pardalos, P.M., Pitsoulis, L.S., Resende, M.G.C.: GRASP with path relinking for the weighted MAXSAT problem. J. Exp. Algorithm. 11, 1-16 (2006)

20. Fukunaga, A., Rabideau, G., Chien, S.: Robust local search for spacecraft operations using adaptive noise. In: Proceedings of the 4th International Workshop on Planning and Scheduling for Space (IWPSS-04). Darmstadt, Germany (2004)

21. Galán, S.F., Mengshoel, O.J.: Constraint handling using tournament selection: Abductive inference in partly deterministic Bayesian networks. Evol. Comput. 17(1), 55-88 (2009)

22. Gallager, R.G.: Low density parity check codes. IRE Trans. Inf. Theory 8, 21-28 (1962)

23. Geman, S., Geman, D.: Stochastic relaxation, Gibbs distributions, and the Bayesian restoration of images. IEEE Trans. Pattern Anal. Mach. Intell. 6(1), 721-741 (1984)

24. Glover, F., Laguna, M.: Tabu search. In: Modern Heuristic Techniques for Combinatorial Problems, pp. 70-150. Wiley, New York (1993)

25. Gomes, C.P., Selman, B.: Algorithm portfolios. Artif. Intell. 126(1-2), 43-62 (2001)

26. Gomes, C.P., Selman, B., Kautz, H.: Boosting combinatorial search through randomization. In: Proceedings of the Fifteenth National Conference on Artificial Intelligence (AAAI-98), pp. 431437. Madison, WI (1998)

27. Gu, P.W., Purdom, J., Franco, J., Wah, B.W.: Satisfiability Problem: Theory and Applications, chapter Algorithms for the Satisfiability SAT Problem: A Survey, pp. 19-152. DIMACS Series in Discrete Mathematics and Theoretical Computer Science. American Mathematical Society, Boston (1997)

28. Henrion, M.: Propagating uncertainty in Bayesian networks by probabilistic logic sampling. In: Uncertainty in Artificial Intelligence 2, pp. 149-163. Elsevier, Amsterdam (1988)

29. Heras, F., Larrosa, J., Oliveras, A.: MiniMaxSAT: an efficient weighted Max-SAT solver. J. Artif. Intell. Res. 31, 1-32 (2008)

30. Hooker, J.: Testing heuristics: We have it all wrong. J Heuristics 1, 33-42 (1996)

31. Hoos, H.H.: An adaptive noise mechanism for WalkSAT. In: Proceedings of the Eighteenth National Conference on Artificial Intelligence (AAAI-02), pp. 655-660. Edmonton, Alberta, Canada (2002)

32. Hoos, H.H.: A mixture-model for the behaviour of SLS algorithms for SAT. In: Proceedings of the Eighteenth National Conference on Artificial Intelligence (AAAI-02), pp. 661-667. Edmonton, Alberta, Canada (2002)

33. Hoos, H.H., Stützle, T.: Towards a characterisation of the behaviour of stochastic local search algorithms for SAT. Artif. Intell. 112(1-2), 213-232 (1999)

34. Hoos, H.H., Stützle, T.: Local search algorithms for SAT: An empirical evaluation. J. Autom. Reason. 24(4), 421-481 (2000)

35. Hoos, H.H., Stützle, T.: Stochastic Local Search: Foundations and Applications. Morgan Kaufmann, San Francisco (2005)

36. Horvitz, E., Ruan, Y., Gomes, C., Kautz, H., Selman, B., Chickering, D.: A Bayesian approach to tackling hard computational problems. In: Proceedings of the 17th Annual Conference on Uncertainty in Artificial Intelligence (UAI-01), pp. 235-244. Seattle, WA (2001)

37. Horvitz, E.J., Suermondt, H.J., Cooper, G.F.: Bounded conditioning: Flexible inference for decisions under scarce resources. In: Proceedings of the Fifth Conference on Uncertainty in Artificial Intelligence (UAI-89), pp. 182-193. Morgan Kaufmann, Windsor (1989)

38. Huberman, B.A., Lukose, R.M., Hogg, T.: An economics approach to hard computational problems. Science 275(3), 51-54 (1997)

39. Hutter, F., Hoos, H.H., Stützle, T.: Efficient stochastic local search for MPE solving. In: Proceedings of the Nineteenth International Joint Conference on Artificial Intelligence (IJCAI05), pp. 169-174. Edinburgh, Scotland. (2005)

40. Jensen, F.V., Lauritzen, S.L., Olesen, K.G.: Bayesian updating in causal probabilistic networks by local computations. SIAM J. Comput. 4, 269-282 (1990)

41. Kask, K., Dechter, R.: Stochastic local search for Bayesian networks. In: Proceedings Seventh International Workshop on Artificial Intelligence and Statistics. Morgan Kaufmann, Fort Lauderdale (1999)

42. Kautz, H., Selman, B., Jiang, Y.: A general stochastic approach to solving problems with hard and soft constraints. In: Gu, J., Pardalos, P.M., Du, D. (eds.) The Satisfiability Problem: Theory and Applications (DIMACS Workshop). American Mathematical Society, Boston (1997)

43. Lagoudakis, M.G., Littman, M.L.: Algorithm selection using reinforcement learning. In: Proc. 17th International Conf. on Machine Learning, pp. 511-518. Morgan Kaufmann, San Francisco (2000) 
44. Larrosa, J., Heras, F., de Givry, S.: A logical approach to efficient Max-SAT solving. Artif. Intell. 172(2-3), 204-233 (2008)

45. Lauritzen, S., Spiegelhalter, D.J.: Local computations with probabilities on graphical structures and their application to expert systems (with discussion). J. R. Stat. Soc. Ser. B 50(2), 157-224 (1988)

46. Li, C.M., Manya, F., Planes, J.: New inference rules for Max-SAT. J. Artif. Intell. Res. 30, 321-359 (2007)

47. Li, Z., D'Ambrosio, B.: Efficient inference in Bayes nets as a combinatorial optimization problem. Int. J. Approx. Reason. 11(1), 55-81 (1994)

48. Lin, R., Galper, A., Shachter, R.: Abductive inference using probabilistic networks: Randomized search techniques. Technical Report KSL-90-73, Knowledge Systems Laboratory, Stanford, November (1990)

49. MacKay, D.J.C.: Information Theory, Inference and Learning Algorithms. Cambridge University Press, Cambridge (2002)

50. Marinescu, R., Dechter, R.: AND/OR branch-and-bound for graphical models. In: Proc. of the 19th International Joint Conference on Artificial Intelligence (IJCAI-05), pp. 224-229 (2005)

51. Marinescu, R., Dechter, R.: Memory intensive branch-and-bound search for graphical models. In: Proc. of the 21st National Conference on Artificial Intelligence (AAAI-06), pp. 1200-1205 (2006)

52. Marinescu, R., Dechter, R.: Best-first AND/OR search for maximum probable explanations. In: Proc. of the 23rd Conference on Uncertainty in Artificial Intelligence (UAI-07) (2007)

53. McAllester, D., Selman, B., Kautz, H.: Evidence for invariants in local search. In: Proceedings of the 14th National Conference on Artificial Intelligence (AAAI-97), pp. 321-326. Providence, RI (1997)

54. Mengshoel, O.J.: Efficient Bayesian Network Inference: Genetic Algorithms, Stochastic Local Search, and Abstraction. Ph.D. thesis, Department of Computer Science, University of Illinois at Urbana-Champaign, Urbana, IL (1999)

55. Mengshoel, O.J.: Designing resource-bounded reasoners using Bayesian networks: system health monitoring and diagnosis. In: Proceedings of the 18th International Workshop on Principles of Diagnosis (DX-07), pp. 330-337. Nashville, TN (2007)

56. Mengshoel, O.J.: Macroscopic models of clique tree growth for Bayesian networks. In: Proceedings of the Twenty-Second National Conference on Artificial Intelligence (AAAI-07), pp. 12561262. Vancouver, British Columbia (2007)

57. Mengshoel, O.J.: Understanding the role of noise in stochastic local search: analysis and experiments. Artif. Intell. 172(8-9), 955-990 (2008)

58. Mengshoel, O.J., Darwiche, A., Cascio, K., Chavira, M., Poll, S., Uckun, S.: Diagnosing faults in electrical power systems of spacecraft and aircraft. In: Proceedings of the Twentieth Innovative Applications of Artificial Intelligence Conference (IAAI-08), pp. 1699-1705. Chicago, IL (2008)

59. Mengshoel, O.J., Darwiche, A., Uckun, S.: Sensor validation using Bayesian networks. In: Proceedings of the 9th International Symposium on Artificial Intelligence, Robotics, and Automation in Space (iSAIRAS-08) (2008)

60. Mengshoel, O.J., Roth, D., Wilkins, D.C.: Stochastic greedy search: Computing the most probable explanation in Bayesian networks. Technical Report UIUCDCS-R-2000-2150, Department of Computer Science, University of Illinois at Urbana-Champaign, Urbana, IL (2000)

61. Mengshoel, O.J., Wilkins, D.C., Roth, D.: Controlled generation of hard and easy Bayesian networks: impact on maximal clique tree in tree clustering. Artif. Intell. 170(16-17), 1137-1174 (2006)

62. Mengshoel, O.J., Wilkins, D.C., Roth, D.: Initialization and restart in stochastic local search: computing a most probable explanation in Bayesian networks. IEEE Trans. Knowl. Data Eng., (in press, 2010)

63. Mills, P., Tsang, E.: Guided local search for solving SAT and weighted MAX-SAT problems. J. Autom. Reason. 24(1-2), 205-223 (2000)

64. Mitchell, D., Selman, B., Levesque, H.J.: Hard and easy distributions of SAT problems. In: Proceedings of the Tenth National Conference on Artificial Intelligence (AAAI-92), pp. 459465. San Jose, CA (1992)

65. Musliner, D., Hendler, J., Agrawala, A.K., Durfee, E., Strosnider, J.K., Paul, C.J.: The challenges of real-time AI. IEEE Comput. 28, 58-66 (1995)

66. Park, J.: Using weighted MAX-SAT engines to solve MPE. In: Proceedings of the 18th National Conference on Artificial Intelligence (AAAI-02), pp. 682-687. Edmonton, Canada (2004) 
67. Park, J.D., Darwiche, A.: Approximating MAP using local search. In: Proceedings of the Seventeenth Conference on Uncertainty in Artificial Intelligence (UAI-01), pp. 403-410. Seattle, WA (2001)

68. Park, J.D., Darwiche, A.: Complexity results and approximation strategies for MAP explanations. J. Artif. Intell. Res. (JAIR) 21, 101-133 (2004)

69. Parkes, A.J., Walser, J.P.: Tuning local search for satisfiability testing. In: Proceedings of the Thirteenth National Conference on Artificial Intelligence (AAAI-96), pp. 356-362. Portland, OR (1996)

70. Pearl, J.: A constraint - propagation approach to probabilistic reasoning. In: Kanal, L.N., Lemmer, J.F., (eds.) Uncertainty in Artificial Intelligence, pp. 357-369. Elsevier, Amsterdam (1986)

71. Pearl, J.: Probabilistic Reasoning in Intelligent Systems: Networks of Plausible Inference. Morgan Kaufmann, San Mateo (1988)

72. Roth, D.: On the hardness of approximate reasoning. Artif. Intell. 82, 273-302 (1996)

73. Ruan, Y., Horvitz, E., Kautz, H.: Restart policies with dependence among runs: a dynamic programming approach. In: Proceedings of the Eighth International Conference on Principles and Practice of Constraint Programming, pp. 573-586. Ithaca, NY (2002)

74. Sang, T., Beame, P., Kautz, H.: A dynamic approach for MPE and weighted MAX-SAT. In: Proceedings of the Twentieth International Joint Conference on Artificial Intelligence (IJCAI07), pp. 173-179 (2007)

75. Schuurmans, D., Southey, F.: Local search characteristics of incomplete SAT procedures. Artif. Intell. 132(2), 121-150 (2001)

76. Selman, B., Kautz, H.: Domain-independent extensions to GSAT: Solving large structured satisfiability problems. In: Proceedings of the International Joint Conference on Artificial Intelligence (IJCAI-93), pp. 290-295. Chambery, France (1993)

77. Selman, B., Kautz, H.A., Cohen, B.: Noise strategies for improving local search. In: Proceedings of the Twelfth National Conference on Artificial Intelligence (AAAI-94), pp. 337-343. Seattle, WA (1994)

78. Selman, B., Levesque, H., Mitchell, D.: A new method for solving hard satisfiability problems. In: Proceedings of the Tenth National Conference on Artificial Intelligence (AAAI-92), pp. 440446. San Jose, CA (1992)

79. Shenoy, P.P.: A valuation-based language for expert systems. Int. J. Approx. Reason. 5(3), 383411 (1989)

80. Shimony, E.: Finding MAPs for belief networks is NP-hard. Artif. Intell. 68, 399-410 (1994)

81. Shwe, M.A., Middleton, B., Heckerman, D.E., Henrion, M., Horvitz, E.J., Lehmann, H.P., Cooper, G.F.: Probabilistic diagnosis using a reformulation of the INTERNIST-1/QMR knowledge base: the, I., probabilistic model and inference algorithms. Methods Inf. Med. 30(4), 241-255 (1991)

82. Viterbi, A.J.: Error bounds for convolutional codes and an asymptotically optimal decoding algorithm. IEEE Trans. Inf. Theory 13, 260-269 (1967)

83. Xing, Z., Zhang, W.: MaxSolver: an efficient exact algorithm for (weighted) maximum satisfiability. Artif. Intell. 164(1-2), 47-80 (2005)

84. Xu, L., Hutter, F., Hoos, H.H., Leyton-Brown, K.: SATzilla-07: The design and analysis of an algorithm portfolio for sat. In: Proceedings of the 13th International Conference on Principles and Practice of Constraint Programming (CP-07) (2007)

85. Zhang, N.L., Poole, D.: Exploiting causal independence in Bayesian network inference. J. Artif. Intell. Res. 5, 301-328 (1996) 